\title{
Using Muonic X Rays to Interrogate Special Nuclear Materials
}

by

Trevor Burn

A thesis submitted to the Faculty of Graduate and Postdoctoral Affairs in partial fulfillment of the requirements for the degree of

Master of Science

in

Physics

Ottawa-Carleton Institute for Physics

Department of Physics

Carleton University

Ottawa, Ontario

(C) 2014

Trevor Burn 


\begin{abstract}
This thesis reports the use and analysis of gamma-ray spectrometry to identify several peaks from a muon beam hitting a target of ${ }^{238} \mathrm{U}$. Two candidates for muonic $\mathrm{x}$ rays occurring from fission products were also identified. A $2 p \rightarrow 1 s \mu^{-} \mathrm{x}$ ray from ${ }^{98} \mathrm{Mo}$ at $(2738.0 \pm 0.7) \mathrm{keV}$ with a yield of $(0.06 \pm 0.02) \mathrm{x}$ ray transitions per muon stop. A $2 p \rightarrow 1 s \mu^{-} \mathrm{x}$ ray from ${ }^{133} \mathrm{Cs}$ at $(3900.2 \pm 1.7) \mathrm{keV}$ with a yield of $(0.04 \pm 0.04) \mathrm{x}$ ray transitions per muon stop.

The equivalent dose from muons was calculated using a simulation of the Bethe-Bloch equation. An upper limit of $1.4 \times 10^{11}$ muons was calculated as the maximum number of muon stops before hitting an equivalent dose limit of $1 \mathrm{mSv}$ in a human phantom.
\end{abstract}

A minimum detectable transition rate of $4.29 \times 10^{5} \times$ ray transitions per second was calculated for the strongest $\mu^{-238} \mathrm{U}$ x ray. 


\section{Acknowledgements}

I would like to thank my supervisor Dr. T.J. Stocki for his patience, guidance, and support for these past few years. His assistance has been essential to the production of this work.

I would also like to thank Dr. Paul Johns for his advice and feedback on this thesis. His guidance is greatly appreciated.

I would also like to thank my family, including my wife, Stephanie Kramer, and my parents. Without their support and understanding, this research would not have been possible.

Thanks goes out to Ernst Esch, Nate Hoteling, Trevor Stocki, Robert Heffner, Haruo Miyadera, Andrew Green, and Andrew Jason for performing the experiment and putting in the hours to collect the data.

In addition to this, I would like to thank my thesis committee, Dr. Paul Johns, Dr. Steven McGarry, Dr. Gerald Oakham, and Dr. Zbigniew Stadnik.

Finally, I'd like to thank all of my professors and colleagues at Carleton University over the past several years. Your instruction and guidance has brought me here today, and I appreciate every moment of it. 


\section{Contents}

Abstract $\quad$ i

Acknowledgements $\quad$ ii

$\begin{array}{ll}\text { Contents } & \text { iii }\end{array}$

List of Tables $\quad$ vii

List of Figures $\quad$ ix

List of Abbreviations $\quad$ xi

1 Introduction and Theory 1

1.1 Nuclear Forensics . . . . . . . . . . . . . . . . . . . . . . . . . 2

1.2 Muon Dose Rate . . . . . . . . . . . . . . . . . . . 4

1.2.1 Kerma, Absorbed Dose, and Dose Equivalent . . . . . . . . . 4

1.3 Muon Theory ........................ 5

1.4 Exotic Atoms . . . . . . . . . . . . . . . . . . 6

1.5 Muonic X Rays . . . . . . . . . . . . . . . . . . . . . . . . . 7

1.6 Muon Capture . . . . . . . . . . . . . . . . . . . . 8

1.6.1 Muon Capture on Heavy Nuclei . . . . . . . . . . . . . 8

1.7 Weak Nuclear Force . . . . . . . . . . . . . . . . . . 9

$1.8 \gamma$ Radiation From Radioactive Decay . . . . . . . . . . . . . . . 11 
1.8.1 $\gamma$ Radiation from other Sources . . . . . . . . . . . . 12

1.9 Photon Interaction with Matter $\ldots \ldots \ldots \ldots \ldots \ldots$

1.9 .1 Photoelectric Effect . . . . . . . . . . . . . . . . 12

1.9 .2 Compton Scattering _ . . . . . . . . . . . . . 13

1.9.3 Electron-Positron Pair Production . . . . . . . . . . . . . 13

1.10 Fission . . . . . . . . . . . . . . . . . . . . 14

1.10 .1 Muon Induced Fission $\ldots \ldots \ldots$

1.11 Thesis Structure . . . . . . . . . . . . . . . . . . 17

1.12 Statement of Contribution . . . . . . . . . . . . 17

2 Related Work 19

2.1 Cosmic Ray Inspection and Passive Tomography (CRIPT) $\ldots \ldots \ldots$

2.2 Muon Induced Fission in Uranium . . . . . . . . . . . . . . . 20

2.3 Radiation Weighting Factor for Slow Negative Muons . . . . . . . 20

2.4 Measurement of Muonic Hyperfine Transition Rates and Muon Capture Yields in Light Nuclei . . . . . . . . . . . . . . . . . 21

3 Previous Work on the Project 23

3.1 Muon Beam Production . . . . . . . . . . . . . . . . 23

3.1 .1 The Beam Telescope . . . . . . . . . . . . . . . . . 24

3.2 Gamma Ray Experiment . . . . . . . . . . . . . . . . 26

3.2 .1 Sources and Targets Used . . . . . . . . . . . . . 26

3.2 .2 Electronics . . . . . . . . . . . . . . . . . . . . . . . 28

3.2 .3 Data Acquisition . . . . . . . . . . . . . . . 30

3.3 Muon Interaction with Natural Chlorine _ . . . . . . . . . . 30

3.3 .1 Acceptances . . . . . . . . . . . . . . . . . . 30

3.3 .2 Chlorine Spectrum Analysis _ . . . . . . . . . . . . . 33

4 Data Analysis $\quad 34$ 
4.1 Energy Calibration . . . . . . . . . . . . . . . . . . . 34

4.1.1 FitzPeaks Gamma Analysis Software . . . . . . . . . . . 35

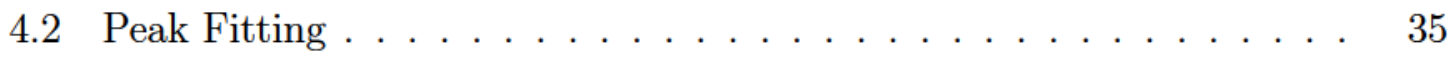

$4.3 \mu^{-} \mathrm{U}$ X Rays . . . . . . . . . . . . . . . . . . . 36

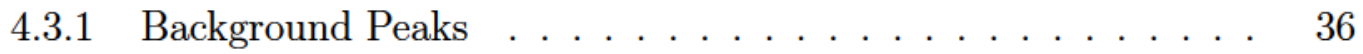

4.3.2 Fission Products . . . . . . . . . . . . . . . . . . 37

4.3.3 Gamma Rays from Muonic Nuclear Capture . . . . . . . . . . 39

4.4 EGSnrc simulation of acceptances . . . . . . . . . . . 40

4.5 Dose from Muons . . . . . . . . . . . . . . . . . . . . . . . . 41

5 Results 45

5.1 Energy Calibration . . . . . . . . . . . . . . . 45

5.2 X Rays and $\gamma$ Rays After Muon Capture on Uranium . . . . . . . 46

5.2 .1 Muonic X Rays from Fission Products . . . . . . . . . . . . 53

5.3 Acceptances . . . . . . . . . . . . . . . . . . 54

5.4 Dose from Muons . . . . . . . . . . . . . . . . . . . 56

6 Discussion 63

6.1 Peak Identification $\ldots \ldots \ldots$

$6.1 .1{ }^{238} \mathrm{U} \alpha$ Decay . . . . . . . . . . . . . . . . . . . 64

$6.1 .2 \mu^{-}$X Ray Candidates $\ldots \ldots \ldots \ldots$. . . . . . . 64

6.1 .3 Yield . . . . . . . . . . . . . . . . . . . . . . 69

6.2 Nuclear Forensics . . . . . . . . . . . . . . . . . 70

6.3 Acceptances . . . . . . . . . . . . . . . . . 71

6.4 Dose from Muons . . . . . . . . . . . . . . . . . . . . . . 72

$\begin{array}{lll}7 & \text { Conclusions and Future Work } & 74\end{array}$

$\begin{array}{ll}\text { References } & 76\end{array}$ 


\section{List of Tables}

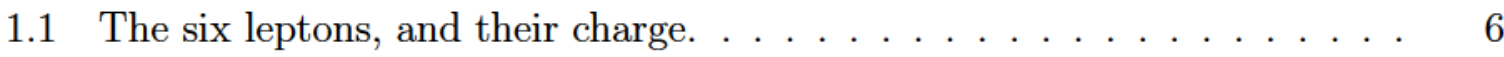

3.1 Some of the various sources and targets used during the experiment. Others were used, but only runs involving these materials were analyzed for

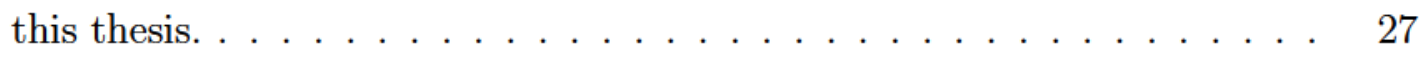

3.2 Energies used for energy calibration by Jason Olsthoorn. . . . . . . . . 30

3.3 The measured acceptances as calculated by Olsthoorn. . . . . . . . . . 32

4.1 Background peaks identified in both uranium runs, with and without muons incident. . . . . . . . . . . . . . . . 38

4.2 The yield of fission products of ${ }^{238} \mathrm{U}$. Uncertainties were not available. . . 39

4.3 Energies of the $2 p_{3 / 2} \rightarrow 1 s_{1 / 2}$ muonic x-ray transitions in various elements and isotopes. Energies were not provided for $Z=44,54$ as they were not

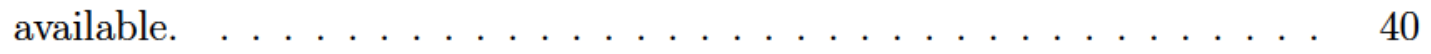

5.1 Sources used for energy calibration, their energies, and respective channels. $\varepsilon$ is electron capture. . . . . . . . . . . . . . . . . 46

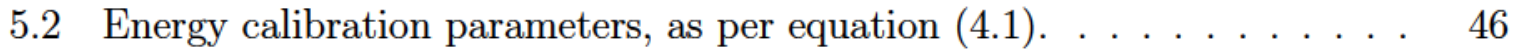

5.3 Peaks associated only with the active-beam run. All peaks in common with the background run have not been included in this table. . . . . . 49

$5.4 \mu^{-} \mathrm{X}$ rays identified from uranium. $\ldots \ldots \ldots \ldots \ldots \ldots$ 
5.5 Candidate muonic $\mathrm{x}$ rays identified from uranium fission products. Upper limits are given for $\mathrm{x}$ rays where no clear peak was identifed. . . . . . . 54

5.6 Simulation acceptance and comparison with experimental acceptance. No electron veto scintillator was present. . . . . . . . . . . . . . 57

5.7 Simulation acceptance and comparison with experimental acceptance. An electron veto scintillator of depth $0.15875 \mathrm{~cm}$ was present in the model. . 58

5.8 Simulation acceptance and comparison with experimental acceptance. An electron veto scintillator of depth $0.3175 \mathrm{~cm}$ was present in the model. . .

5.9 Simulation acceptance and comparison with experimental acceptance. An electron veto scintillator of depth $0.5 \mathrm{~cm}$ was present in the model. . . . .

5.10 Energy deposited into a phantom by one muon for a given energy at a given maximum path length. . . . . . . . . . . . . . 61

5.11 The dose equivalent that one muon of a given energy at a maximum path length would deposit into a human of mass $70 \mathrm{~kg} . \ldots \ldots \ldots$. . . . .

5.12 The number of muon stops of a given energy at a maximum path length required to attain a dose equivalent of $1 \mathrm{mSv}$ in the same human phantom.

6.1 Predicted number of counts from the "existing fission product" method of obtaining muonic $\mathrm{x}$ rays from fission products. . . . . . . . . . 68

6.2 Predicted number of counts from the "muon transfer" method of obtaining muonic $\mathrm{x}$ rays from fission products. . . . . . . . . . . 69

6.3 Yield (x ray transitions per muon stop) for the muonic $2 p \rightarrow 1 s$ x-ray

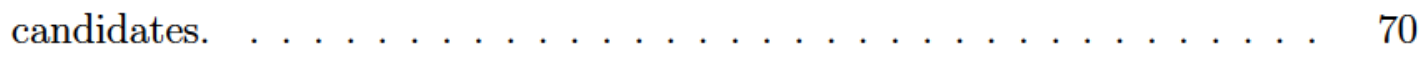

6.4 The sum of the number of sigma difference for all four scintillator thicknesses. 73 


\section{List of Figures}

1.1 The distribution of muonic X-ray energies for ${ }^{235} \mathrm{U}$ and ${ }^{238} \mathrm{U} \ldots \ldots . . . \quad 3$

1.2 First order Feynman diagram of beta decay. At the bottom is a neutron $(u d d)$, and at the top is a proton (uud), an electron $\left(e^{-}\right)$and an electron anti-neutrino $\left(\bar{\nu}_{e}\right) \ldots \ldots \ldots \ldots \ldots \ldots \ldots \ldots \ldots \ldots \ldots \ldots \ldots \ldots \ldots \ldots$

1.3 First order Feynman diagram of muon decay. . . . . . . . . . . 10

1.4 First order Feynman diagram of muon capture. At the bottom is a neutron $(u d d)$ and a muon $\mu^{-}$, and at the top is a proton (uud) and a muon anti-

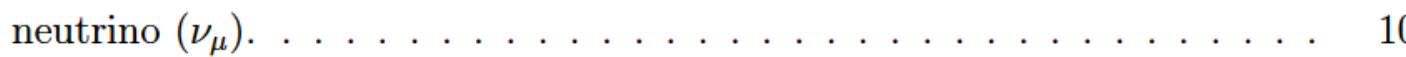

1.5 First order Feynman diagram of electron-positron pair production. Not shown is the nucleus necessary for conservation of momentum. . . . . 15

3.1 The various beamlines at TRIUMF. . . . . . . . . . . . . . 25

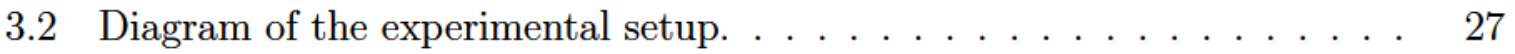

3.3 Schematics for the electronics for the experiment. . . . . . . . . . . . 29

4.1 Geometry used in the acceptance simulation. The germanium detector is surrounded by air and aluminum. All distances are given in $\mathrm{cm}$. For the $\mu^{-} \mathrm{Au} \times$ rays, $0.1 \mathrm{~cm}$ of gold replaces the first $0.1 \mathrm{~cm}$ of air at the top. .

5.1 The full ${ }^{238} \mathrm{U}$ spectrum. . . . . . . . . . . . . . . . . . . 48

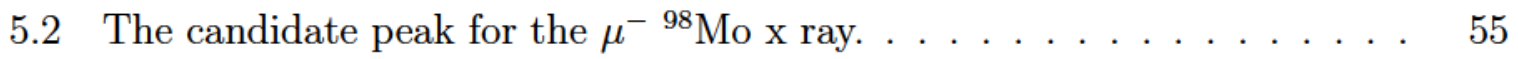


5.3 The candidate for the $\mu^{-}{ }^{137} \mathrm{Cs} \mathrm{x}$ ray. The "peak" is not statistically

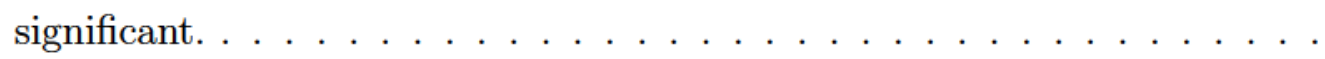

5.4 Experimental and simulated acceptances for the germanium detector. ${ }^{22} \mathrm{Na}$, ${ }^{60} \mathrm{Co},{ }^{133} \mathrm{Ba},{ }^{152} \mathrm{Eu}$ were the gamma sources used for calibration, and $\mu^{-} \mathrm{x}$ rays from gold were used for the high energy calibration. No scintillator present in this simulation. . . . . . . . . . . . . . 61

6.1 The ${ }^{238} \mathrm{U}$ decay chain. . . . . . . . . . . . . . . . . . . 65 


\section{List of Abbreviations}

BGO Bismuth Germanium Oxide

CERN European Organization for Nuclear Research

CRTI Chemical Biological Radionuclear Explosive Research Technology Initiative

DE Double Escape

EC Electron Capture

EGSnrc Electron and Gamma Shower National Research Canada

GEANT4 GEometry ANd Tracking version 4

HPGe High Purity Germanium

ICRU International Commission on Radiation Units

MCNPX Monte Carlo N-Particle Extended

MDA Minimum Detectable Activity

SE Single Escape

SNM Special Nuclear Materials

TRIUMF TRI-University Meson Facility

VME Virtual Machine Environment 


\section{Chapter 1}

\section{Introduction and Theory}

Special nuclear materials (SNM) are of great interest to border security. As the threat of terrorism keeps increasing, the likelihood that someone attempts to perpetrate an attack using nuclear materials does as well. Thus, being able to identify special nuclear materials becomes more important.

This thesis is done as part of CRTI (Chemical Biological Radionuclear Explosive Research Technology Initiative) study RN-091AP - Active Muon Interrogation to find hidden Special Nuclear Material, led by Trevor J. Stocki at Health Canada. The purpose of this study within the project was to evaluate the feasibility of active muon interrogation to help detect smuggled special nuclear materials. It was also proposed that this method could be used for standoff detection (detection at a distance) of SNM. The main goal of this project is to use muonic $\mathrm{x}$ rays to detect SNM. Future work would include miniaturizing the accelerator necessary for muon beam production, which would have a number of spin off benefits for high energy physics and $\mu \mathrm{SR}$ (muonic Spin Resonance).

Previously, experiments were done at the TRIUMF (TRI-University Meson Facility) National Lab in Vancouver, BC by Stocki, Jason, et. al. [1]. This thesis is an analysis of the data produced during that experiment. 
Active muon interrogation is being investigated because it has several features that make it attractive. These include the fact that muons penetrate most matter with little nuclear reaction until slowed by ionization losses in a small distance, and that distinct high-energy $\mathrm{x}$ rays are produced in high $\mathrm{Z}$ materials (such as uranium). The method of this thesis would not be the one to be used in an attempt to search for SNM. A more likely method would be the one proposed by Jason et. al. to use an accelerator on a large ship with a helicopter-borne detection system [2].

There are three main purposes of this thesis. First, is to analyze the muonic uranium spectrum, with an emphasis on searching for muonic $\mathrm{x}$ rays from both uranium and the fission products of uranium. Muonic $\mathrm{x}$ rays from fission fragments have not been observed [3], and their discovery would add extra signals to help detect SNM. This would especially be useful since acceptance is higher at lower energies, making these signals easier to detect. The second purpose is to verify the acceptance of the detector as calculated by Jason Olsthoorn [4]. A previous group member attempted to calculate this using the MCNPX Monte Carlo simulation tool, but was unable to do so with acceptable accuracy. Finally, in this thesis the calculation of an estimated upper limit (the number of muons that would guarantee that equivalent dose) on the number of muons entering a human body required to produce an equivalent dose of $1 \mathrm{mSv}$ (the dose limit set by the Government of Canada for a non-radiation worker in a year [5]) is outlined.

\section{$1.1 \quad$ Nuclear Forensics}

Nuclear forensics refers to several techniques used to identify the origin of nuclear materials. Any process undertaken upon nuclear materials will result in a signature that can be tracked. These processes can be physical or chemical. There are several reasons to want to identify nuclear materials, including non-proliferation, nuclear 
safeguards, and environmental samples [6]. Several different nuclear forensics methods exist, and consist of measuring different signatures. Roughly speaking, these fall into five different categories: radiological, physical characterization, traditional forensic analysis, isotope analysis, and chemical analysis. The method described in this thesis can be used for nuclear forensics.

One of the advantages that active muon interrogation provides is the ability to identify isotopes of SNM. At high Z, isotopes give off $\mathrm{x}$ rays unique to that particular isotope, and so it is possible to identify the mixture of isotopes based on the spectrum produced. For example, ${ }^{235} \mathrm{U}$ and ${ }^{238} \mathrm{U}$ are distinguishable from their muonic X-ray spectra, as can be seen in Figure 1.1.

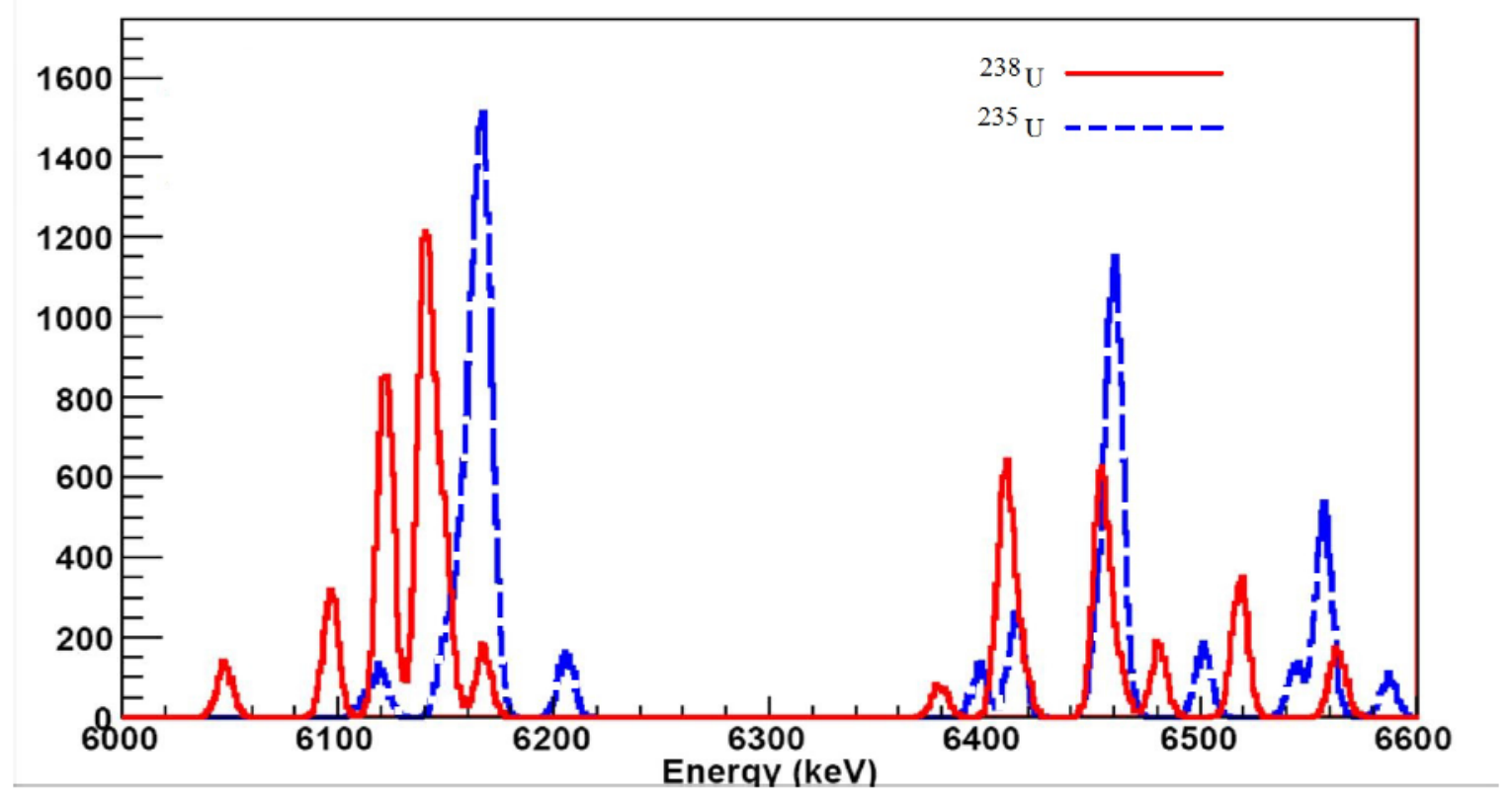

Figure 1.1: Distribution of muonic $x$ ray energies and intensities for ${ }^{235} \mathrm{U}$ and ${ }^{238} \mathrm{U}$. Intensities and energies for the ${ }^{238} \mathrm{U}$ peaks are from experiment, intensities and eneriges for the ${ }^{235} \mathrm{U}$ come from Close et. al.[7]. Graphic used with permission of Andrew Jason [8]. 


\subsection{Muon Dose Rate}

If active muons were ever to be used for border security (as proposed by Andrew Jason [2]), it is possible that humans would be in the path of these muons. Thus, knowing the equivalent dose rate of the muon beam is important in order to ensure the safety of both security workers and persons in the vicinity of a target of interest.

\subsubsection{Kerma, Absorbed Dose, and Dose Equivalent}

Dose is a measure of how much energy is deposited by ionizing radiation in a medium per unit mass. There are three related measures with respect to this concept: kerma, absorbed dose, and dose equivalent.

Kerma stands for Kinetic Energy Released per unit MAss, and is defined as

$$
K=\frac{\mathrm{d} E_{\mathrm{tr}}}{\mathrm{d} m}
$$

where $\mathrm{d} E_{\mathrm{tr}}$ is the energy transfered into a mass $\mathrm{d} m$ [9]. It carries the units of $\mathrm{J} / \mathrm{kg}$.

Absorbed dose is defined as

$$
D=\frac{\mathrm{d} E_{\mathrm{ab}}}{\mathrm{d} m}
$$

where $\mathrm{d} E_{\mathrm{ab}}$ is the energy absorbed by a mass $\mathrm{d} m$. It carries the units of gray, Gy, where $1 \mathrm{~Gy}=1 \mathrm{~J} / \mathrm{kg}$. The difference between kerma and absorbed dose is that while kerma best represents the amount of energy lost by the photon, absorbed dose represents the amount of that energy that is actually absorbed by the medium. It is also important to note that, because the transferred energy may be carried away by ionized electrons, the absorbed dose and kerma occur at different locations. It is usually the case that $E_{\mathrm{tr}}>E_{\mathrm{ab}}$, as some of the energy is radiated away as bremsstrahlung (braking radiation) [9].

Dose equivalent is defined as

$$
H=D \cdot w_{r}
$$


where $D$ is the absorbed dose and $w_{r}$ is a dimensionless radiation weighting factor. The unit of the dose equivalent is the sievert, Sv. Because $w_{r}$ is unitless, $1 \mathrm{~Sv}=1 \mathrm{~Gy}$, but the two are used separately to indicate whether it is absorbed dose or equivalent dose that is being discussed [9]. The radiation weighting factor is dependent on the type of ionization radiation, with $\gamma$ rays and electrons having $w_{r}=1, \alpha$ particles having $w_{r}=20$, and other particles falling somewhere in between. The weighting factor for muons is discussed in section 2.3 .

\subsection{Muon Theory}

The muon is a subatomic particle that was first hypothesized by Yukawa in 1935 [10]. He originally proposed a particle with a mass between that of the proton and electron, and it would be the particle that carries the nuclear force, much as the photon carries the electromagnetic force. Later, several experiments, including those by Anderson and Neddermeyer [11], and Street and Stevenson [12], strongly suggested the existence of a charged particle at the appropriate mass. Later work demonstrated that, although the particle did exist in the correct mass range, it did not exhibit key properties that the Yukawa force carrier would have. In 1947, Lattes et al. found evidence of one cosmic ray particle decaying into another [13] . These were the pion $(\pi)$ and the muon $(\mu)$. We know now that the pion decays as follows

$$
\begin{aligned}
& \pi^{-} \rightarrow \mu^{-} \overline{\nu_{\mu}} \\
& \pi^{+} \rightarrow \mu^{+} \nu_{\mu}
\end{aligned}
$$

where $\nu_{\mu}$ and $\overline{\nu_{\mu}}$ are muon neutrinos and muon anti-neutrinos.

The muon is a lepton ( $\left.\operatorname{spin}{ }^{1} / 2\right)$ with a mass of $(105.6583715 \pm 0.0000035) \mathrm{MeV} / \mathrm{c}^{2}$, a mean free lifetime of $(2.1969811 \pm 0.0000022) \times 10^{-6} \mathrm{~s}$, and a charge of \pm 1 [14] It is one of 6 leptons (see Table 1.1), which are particles that interact via the weak nuclear force, and the electromagnetic force (for particles with electric charge). Free 
muons primarily decay into electrons, via the following process:

$$
\mu^{-} \rightarrow e^{-} \nu_{\mu} \overline{\nu_{e}}
$$

for $\mu^{-}$and

$$
\mu^{+} \rightarrow e^{+} \overline{\nu_{\mu}} \nu_{e}
$$

for $\mu^{+}$. Although neutrinos are known to have non-zero mass, their mass is considered negligible for the work done in this thesis.

Table 1.1: The six leptons, and their charge.

\begin{tabular}{|c|c|c|c|}
\hline Charge & \multicolumn{3}{|c|}{ Particle } \\
\hline \pm 1 & $e$ & $\mu$ & $\tau$ \\
\hline 0 & $\nu_{e}$ & $\nu_{\mu}$ & $\nu_{\tau}$ \\
\hline
\end{tabular}

\subsection{Exotic Atoms}

An exotic atom is an atom in which one or more of the electrons have been replaced by a different negatively charged particle. This can include negatively charged pions $\left(\pi^{-}\right)$, kaons $\left(K^{-}\right)$, muons $\left(\mu^{-}\right)$, anti-protons $(\bar{p})$, and others $\left(\Sigma^{-}, \Omega^{-}, \Xi^{-}\right)$. The first experimental indication of exotic atoms occurred in 1947, by Conversi et al. [15], and was a muonic atom. Further evidence was produced by Chang in 1949 [16], by stopping cosmic ray muons in $\mathrm{Al}, \mathrm{Fe}$, and $\mathrm{Pb}$ foils.

The energy levels of these exotic atoms are nominally organized in the same structure as that of a regular atom, but since the exotic particles are much more massive than the electron, the energy levels are much higher. The orbital distance is also much lower, by a factor of $m_{e} / M$, and thus there can be some physical overlap between the wavefunction of the nucleus and the exotic particle for high $\mathrm{Z}$ elements. This leads to an increase in nuclear interaction. 


\subsection{Muonic X Rays}

Muonic x rays are formed by producing muons, which are fired into a target material. At TRIUMF, for example, the kinetic energy of the incident muons is around $10 \mathrm{MeV}$. It is reduced to $2 \mathrm{keV}$ by electromagnetic interactions within the target material. The muon is then captured by a nearby atom into a high orbital angular momentum state, and hence excited state $(n \approx 14)$. At this point, the muon will cascade into the ground state, usually the $1 s$ state on a timescale of $\sim 10^{-13} \mathrm{~s}[17]$. During the first stage, Auger electrons are produced. These are electrons that absorb the muon's de-excitation energy and leave the atom entirely. At the lower transitions $(n \approx 5$ [17]), x rays are emitted, typically at high energy levels. Those specific energies are given by equation (1.8) below, where $j$ is the total angular momentum, $A$ is the mass number, $\alpha \approx 1 / 137$ is the fine structure constant, and $m_{\mu}$ is the muon mass. The literature values for muonic x-ray energies used later in the thesis come from Engfer et. al. [18] and Close et. al. [7].

$$
E_{n, j}=-\frac{m_{\mu} c^{2}}{1+m_{\mu} / A} \frac{(Z \alpha)^{2}}{2 n^{2}}\left[1+\left(\frac{Z \alpha}{n}\right)^{2}\left(\frac{n}{j+(1 / 2)}-\frac{3}{4}\right)\right]
$$

Equation (1.8) is the same for muons as it is for electrons, except that $m_{\mu}$ is used instead of $m_{e}$. Because of this, muonic $\mathrm{x}$ rays have much higher energies than the traditional electronic $\mathrm{x}$ rays by factor of $m_{\mu} / m_{e} \approx 207$. However, while a muon is in the $1 s$ state, the muon has an orbital radius comparable to the nuclear radius (especially for high $Z$ materials), and so the energy of the muonic x-rays ending in a $1 s$ state will be reduced slightly. After reaching the $1 s$, the muon will either decay into an electron and two neutrinos as in equation (1.6), or it can be captured by the nucleus. 


\subsection{Muon Capture}

If the muon does not decay into an electron, then the muon will be captured by the nucleus. In this case, the muon causes a proton to change into a neutron, with a neutrino being emitted, and the nucleus is left in a highly excited state.

$$
\mu^{-} p \rightarrow n \nu_{\mu}
$$

After nuclear muon capture, the most likely scenario is that the neutron will be emitted from the nucleus. It is also possible for more than one neutron to be emitted, or for charged particles to be emitted as well. The lifetime of the muon in the $1 s$ state is dependent on the nucleus, and increases as $Z$ increases. As the lifetime increases, the rate of nuclear capture does as well. So for high $Z$ materials such as ${ }_{92}^{238} \mathrm{U}$, the muon capture rate is high $\left[(12610 \pm 70) \times 10^{3} \mathrm{~s}^{-1}\right]$, whereas for low $Z$ materials such as ${ }_{4}^{9} \mathrm{Be}$, the muon capture rate is only $(6.1 \pm 0.6) \times 10^{3} \mathrm{~s}^{-1}[17]$.

For example, consider a $\mu^{-}$undergoing nuclear capture on ${ }^{35} \mathrm{Cl}$. This can change it to ${ }^{35} \mathrm{~S}$. At this point, several things can happen. The neutrino could take away a large fraction of the energy, and thus the nucleus de-excites, emitting $\gamma$ rays. Alternatively, the neutrino could leave enough energy for a neutron to be emitted, leaving ${ }^{34} \mathrm{~S}$ to emit $\gamma$ rays.

\subsubsection{Muon Capture on Heavy Nuclei}

Nuclear muon capture has its own set of idiosyncrasies not found in low $A$ materials [17]. As $Z$ increases, the nuclear energy levels are characterized poorly in comparison with their low $Z$ counterparts, and the $\gamma$ ray energies tend to overlap. There are particular patterns with heavy nuclei that tend to be seen. Namely, the nuclear capture can result in 1 to 3 neutrons emitted, with $\gamma$ rays from nuclei resulting from the $1 n$ emission dominant, as well as $\gamma$ rays from the $2 n$ and $3 n$ emissions present. 


\subsection{Weak Nuclear Force}

The weak nuclear force is one of the four fundamental forces, and acts on fermions. Of particular interest to this thesis is that the weak force mediates both muon decay and muon capture. There are three weak force carriers, two charged ( $W^{ \pm}$bosons) and one neutral ( $Z^{0}$ boson). The charged carriers are what enable particles to decay into one another, whereas the neutral carrier enables the uncharged neutrinos to interact with each other (an interaction that is not able to be mediated by other neutral carriers, such as the photon). The $Z^{0}$ boson also allows for weak interactions between charged particles, similar to the photon, but at a reduced strength. The $Z^{0}$ boson also serves to unify the electromagnetic and weak forces. The weak force mediates several reactions, including neutrino interactions and radioactive decay. For example, $\beta^{-}$decay follows the structure seen in Figure 1.2. In $\beta^{-}$decay, a down quark is converted to an up quark, emitting a $W^{-}$boson. The boson then decays into an electron and an electron anti-neutrino.

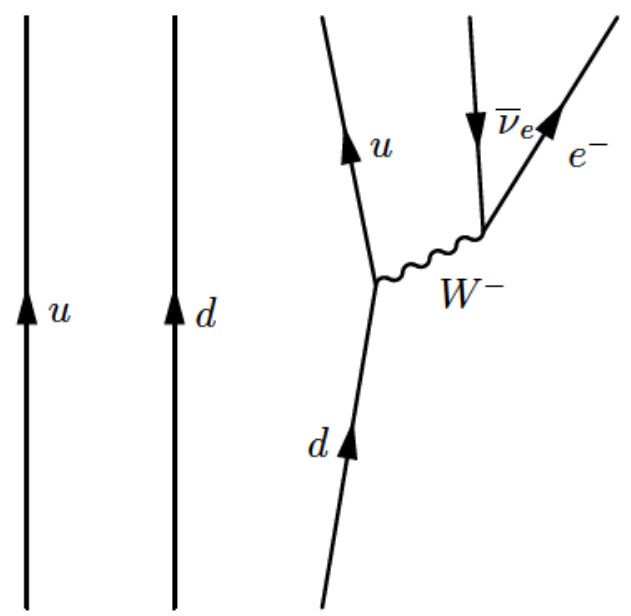

Figure 1.2: First order Feynman diagram of beta decay. At the bottom is a neutron $(u d d)$, and at the top is a proton $(u u d)$, an electron $\left(e^{-}\right)$and an electron anti-neutrino $\left(\bar{\nu}_{e}\right)$.

Muon decay occurs when a muon decays into an electron (or positron) and two neutrinos, as in equation (1.6). This is again mediated by the $W^{-}$boson, as can be 
seen in Figure 1.3. The muon decays into a $W^{-}$and a muon neutrino, and the boson then decays into an electron and electron anti-neutrino (similar to what happens in $\beta^{-}$decay). Muon capture occurs when a muon decays into a $W^{-}$and a $\nu_{\mu}$, but instead of the $W^{-}$decaying into an neutrino-electron pair as in muon decay, the $W^{-}$ changes a $d$ quark into an $u$ quark (and hence changes a proton to a neutron) (see Figure 1.4).

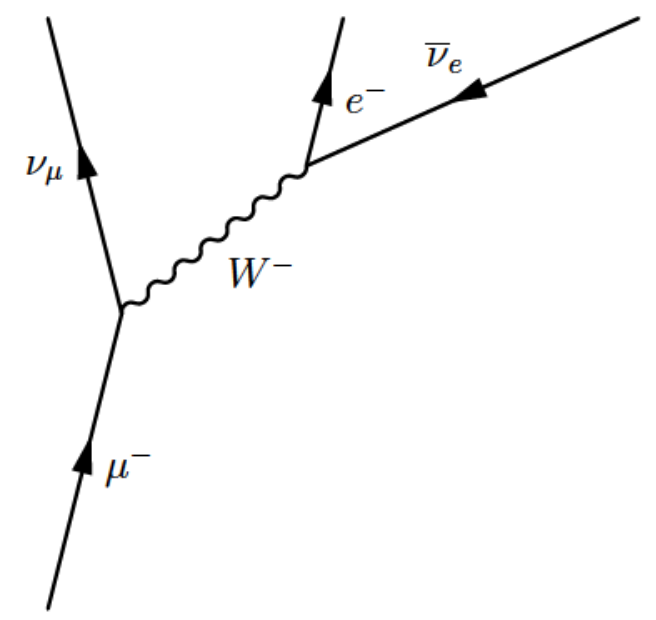

Figure 1.3: First order Feynman diagram of muon decay.
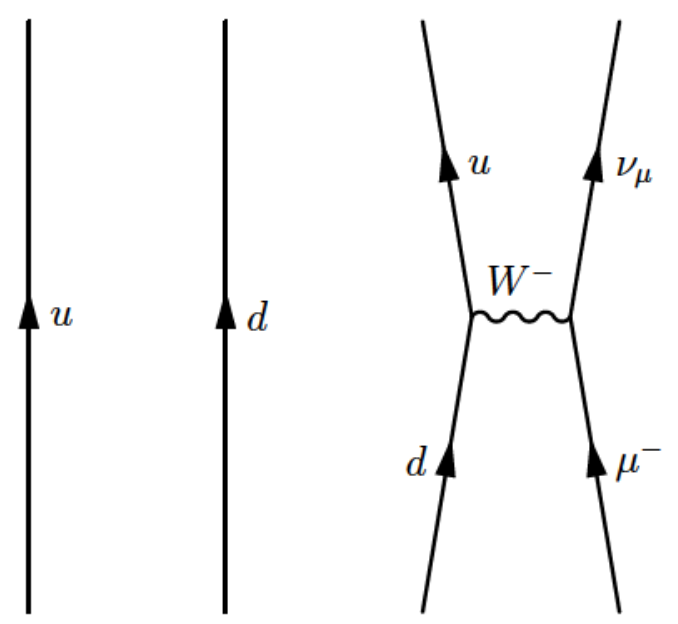

Figure 1.4: First order Feynman diagram of muon capture. At the bottom is a neutron $(u d d)$ and a muon $\mu^{-}$, and at the top is a proton (uud) and a muon anti-neutrino $\left(\nu_{\mu}\right)$. 


\section{8 $\gamma$ Radiation From Radioactive Decay}

Most of the sources and targets used in this project are radioactive. As such, they undergo one of several different types of radioactive decay [19]. These include

- $\beta^{-}$decay, where a neutron becomes a proton, which results in the emission of an electron,

$$
{ }_{Z}^{A} \mathrm{X} \rightarrow{ }_{Z+1}^{A} \mathrm{Y}+\beta^{-}+\bar{\nu}_{e}
$$

- $\beta^{+}$decay, where a proton becomes a neutron, which results in the emission of a positron,

$$
{ }_{Z}^{A} \mathrm{X} \rightarrow{ }_{Z-1}^{A} \mathrm{Y}+\beta^{+}+\nu_{e}
$$

- EC, or electron capture, which absorbs an orbital electron, or

$$
{ }_{Z}^{A} \mathrm{X}+\beta^{-} \rightarrow{ }_{Z-1}^{A} \mathrm{Y}+\nu_{e}
$$

- $\alpha$ decay, which results in the emission of an $\alpha$ particle (2 protons and 2 neutrons).

$$
{ }_{Z}^{A} \mathrm{X} \rightarrow{ }_{Z-2}^{A-4} \mathrm{Y}+\alpha
$$

The energies of these decays exist along a continuum. Because of this, they cannot be readily identified, and are lost in the background radiation. After the decay, some of the energy from the process is carried away by the neutrino (if one exists), but not all, and so the nucleus is left in an excited state. This energy is emitted in the form of $\gamma$ rays of varying energies, according to the energy structure of the nucleus.

It is worth noting that $\mathrm{EC}$ is a very similar process to that of $\mu^{-}$capture, but because the mass of the electron is much smaller than that of the muon, the energy available in EC is much lower than the energy available during the process of $\mu^{-}$ capture. 


\subsection{1 $\gamma$ Radiation from other Sources}

There are other sources of $\gamma$ radiation that can affect the spectrum. Two of these are $(n, \gamma)$ and $\left(n, n^{\prime} \gamma\right)$ events. An $(n, \gamma)$ event occurs when a neutron is captured by a nucleus. These neutrons occur as a result of nuclear muon capture, outlined in section 1.6. In this case, as with $\gamma$ radiation from radioactive decay, the nucleus is left in an excited state. When it de-excites, a $\gamma$ ray is emitted according to the nuclear energy structure. An $\left(n, n^{\prime} \gamma\right)$ event is similar, but a neutron is emitted from the nucleus as well as a $\gamma$ ray.

\subsection{Photon Interaction with Matter}

A gamma ray spectrometer was used to collect the data that was analyzed in this thesis. As such, the interactions of photons with matter are of interest, especially electron-positron pair production. Photons interact with matter in one of four different ways: the photoelectric effect, Compton scattering, electron-positron pair production, and Rayleigh scattering. These first three result in the photon losing energy to the material, whereas Rayleigh scattering is an elastic event (the photon conserves its energy, changing only its direction). Each of these effects (except Rayleigh scattering) dominates at different energy levels. The photoelectric effect typically dominates at low energies, pair production dominates at high energies, and Compton scattering is the main form of interaction for middling energies.

\subsubsection{Photoelectric Effect}

The photoelectric effect is the process of a photon being absorbed by an electron, causing it to escape the atom to which it is bound. The electron cross section for such an event occurring, $\tau_{e}$, is a function of the atomic number of the target, $Z$, and 
the photon's energy $E$, such that

$$
\tau_{e} \propto \frac{Z^{m}}{E^{n}}
$$

where $3 \leq m \leq 3.8$ and $1 \leq n \leq 3[9,19]$.

\subsubsection{Compton Scattering}

Compton scattering is inelastic scattering between a photon and an electron in the target material. The energy gained by the electron $E_{e}$ (or the energy lost by the photon) follows the equation

$$
E_{e}=E_{\gamma}\left\{1-\frac{1}{\left[1+E_{\gamma}(1-\cos \theta) / m_{0} c^{2}\right]}\right\}
$$

where $E_{\gamma}$ is the initial energy of the photon, $\theta$ is the scattering angle, and $m_{0} c^{2}$ is the rest mass of the electron [19]. This equation holds for a free electron, but some binding effects change the relationship for $\theta \approx 0$ [19]. This leads to a range of different scattering energies, from $0 \mathrm{keV}$ for the $0^{\circ}$ case, and a number less than $E_{\gamma}$ for the $180^{\circ}$ case.

Compton scattering has an effect for spectrometry, as it makes it possible for the photon to escape the detector before depositing all of its energy. This produces a background spectrum for the photopeak, which can make peak detection more difficult. One can then introduce a Compton suppressor, or a secondary detector (typically made of BGO (bismuth germanium oxide) or $\mathrm{NaI}(\mathrm{Tl})$ ) surrounding the germanium detector that detects if a photon has escaped the detector before depositing all of its energy. If this occurs, the electronics are set such that any such events will not be registered as part of the spectrum.

\subsubsection{Electron-Positron Pair Production}

When a photon interacts with a nucleus, it has the possibility of undergoing electronpositron pair production. In this event, the photon interacts with the Coulomb field of 
the nucleus, and creates an electron-positron pair, as in Figure 1.5. This only occurs for $E_{\gamma} \geq 1022 \mathrm{keV}$ (i.e. if there is enough energy to create two electrons at rest). It is also possible for the photon to interact with the Coulomb field of the electrons, but this requires a higher minimum energy (4 times the electron rest mass), and the cross-section is much lower. The differential cross section for the interaction with the nucleus is given by

$$
\frac{\mathrm{d} \kappa}{\mathrm{d} \Omega}=\frac{Z^{2}}{137} \frac{r_{0}^{2}}{2 \pi} m_{0}^{2} c^{4} F_{\text {pair }}
$$

where $F_{\text {pair }}$ is an function of the pair's energy, momentum, and angle [9]. The effect is that $\kappa$, the cross section for pair production increases with the energy of the incident photon.

The electron is quickly brought to a stop in the material, and the positron quickly undergoes annihilation with an electron from the material, producing two (nearly) back-to-back $511 \mathrm{keV}$ photons. These photons will then continue through the detector. However, it is possible that one or both of these $511 \mathrm{keV}$ photons escape the detector without depositing their energy. Should this occur, normally the Compton suppressor will detect it, and the event will be discarded. In other cases though, one or both photons will escape without triggering the Compton suppressor. In this case, this will create secondary and tertiary peaks in the gamma-ray spectrum at $E_{\gamma}-511 \mathrm{keV}$ and $E_{\gamma}-1022 \mathrm{keV}$, called the single escape (SE) and double escape (DE) peaks respectively. These both occur at reduced intensity, and are further reduced in intensity with a Compton suppressor.

\subsection{Fission}

Uranium, the target of interest for this thesis, is capable of undergoing nuclear fission. As part of this thesis involves the search for muonic $\mathrm{x}$ rays from fission products, a discussion of fission is necessary to understand how these $\mathrm{x}$ rays can occur. 


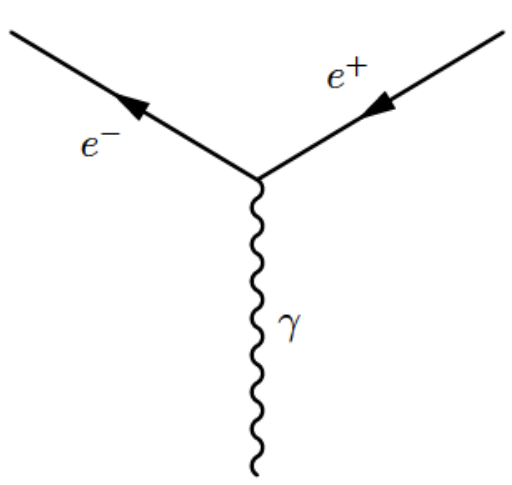

Figure 1.5: First order Feynman diagram of electron-positron pair production. Not shown is the nucleus necessary for conservation of momentum.

Fission is when a nucleus splits into two daughter nuclei, and one or more neutrons. It occurs when the nucleus becomes unstable. One reason for it to become unstable is an excess of either protons or neutrons, giving it more energy than the nuclear binding energy. Another cause is when it gains energy from an outside source, leaving the nucleus in an excited state. Fission will proceed when the daughter nuclei are more stable than the parent nuclei.

Fission can occur through several processes. The first is spontaneous fission, which occurs when a nucleus spontaneously decays into two daughter nuclei. This type of fission only occurs in high $Z$ elements such as ${ }_{92} \mathrm{U}$ or ${ }_{90} \mathrm{Th}$, and typically occurs much less often than other decays for the same isotope. For example, for ${ }^{238} \mathrm{U}$, the branching ratio of $\alpha$ decay to spontaneous fission is $100:(5.45 \pm 0.07) \times 10^{-5}[20]$. An example of spontaneous fission can be seen in equation (1.13).

$$
{ }_{92}^{238} \mathrm{U} \rightarrow{ }_{55}^{133} \mathrm{Cs}+{ }_{37}^{102} \mathrm{Rb}+3 n
$$

The second type of fission is neutron induced fission. Similar to the $(n, \gamma)$ events mentioned in Section 1.8.1, neutron induced fission begins when a neutron is captured by the nucleus. However, instead of the nucleus simply de-exciting and emitting a $\gamma$ ray, the nucleus becomes unstable and instead splits into two daughter nuclei and some (0 or more) neutrons. An example of neutron induced fission can be seen in 
equation (1.14).

$$
n+{ }_{92}^{238} \mathrm{U} \rightarrow{ }_{54}^{136} \mathrm{Xe}+{ }_{38}^{102} \mathrm{Sr}+n
$$

\subsubsection{Muon Induced Fission}

The final type of fission relevant to this thesis is muon induced fission. Muon induced fission itself can occur through two different mechanisms. The first of these is a prompt fission induced by nonradiative transitions. A nonradiative transition occurs when an atomic muon undergoes a transition from one atomic energy level to another (typically the $2 p \rightarrow 1 s$ transition), but instead of emitting an $\mathrm{x}$ ray, the energy is instead transferred to the nucleus, bringing it into an excited state [21]. This then causes the nucleus to undergo fission, as can be seen in equation (1.15).

$$
{ }_{92}^{238} \mathrm{U}^{*} \rightarrow{ }_{54}^{136} \mathrm{Xe}+{ }_{38}^{102} \mathrm{Sr}
$$

The second mechanism of muon induced fission occurs as a result of nuclear muon capture. When the muon is captured by the nucleus, it results in a proton changing into a neutron and emitting a neutrino,

$$
\mu^{-}+p \rightarrow n+\nu_{\mu}
$$

or, in terms of the atoms involved:

$$
\mu^{-}+{ }_{Z}^{A} \mathrm{X} \rightarrow{ }_{Z-1}^{A} \mathrm{Y}+\nu_{\mu} \rightarrow{ }_{Z-1-n}^{A-m} \mathrm{~S}+{ }_{n}^{m} \mathrm{~T}+\nu_{\mu}
$$

Most of the energy of this reaction is transferred to the neutrino, but it does leave the daughter nucleus ${ }_{Z-1}^{A} \mathrm{Y}$ in an excited state with $15 \mathrm{MeV}$ to $20 \mathrm{MeV}$ [21]. For heavy nuclei, like ${ }^{238} \mathrm{U}$, this can be enough energy for fission to occur, for example as in equation (1.18).

$$
\mu^{-}+{ }_{92}^{238} \mathrm{U} \rightarrow{ }_{42}^{100} \mathrm{Mo}+{ }_{49}^{135} \mathrm{In}+3 n+\nu_{\mu}
$$




\subsection{Thesis Structure}

In this thesis, I will cover the analysis of the data produced by the experiment, the simulation of the acceptance, and the dose rate calculations. Chapter 2 covers related work, including work by Dr. Trevor Stocki that provided the basis for the gamma ray spectrometry. Chapter 3 covers the previous work done on the project, including the experimental setup, details about the detector acceptance, and previous analysis of different spectra. Chapter 4 reports my analysis of the data collected from the experiment. I also detail the EGSnrc (Electron and Gamma Shower) simulation of the detector acceptances, and the calculation for the equivalent dose rate from muons. Chapter 5 contains the results of the analysis, including energy calibration, the results of the EGSnrc simulation, an analysis of the $\mu^{-}$uranium spectrum, and the dose rate calculations. Chapter 6 discusses the results found in the previous chapter, leading to a conclusion and discussion of future work in Chapter 7 .

\subsection{Statement of Contribution}

I worked to write a detector geometry for dosrznrc (an EGSnrc user code) to simulate the acceptance of the experimental setup. To do this, I modified an existing geometry written by previous students to match up with the geometry of the experiment. I also simulated several hypothetical geometries when it was discovered that there was a discrepancy in the acceptances resulting from the simulation.

I analyzed the gamma-ray spectra of several different beam runs. I re-examined the energy calibration originally performed by Jason Olsthoorn, and improved it. I performed an analysis of two different runs with uranium as the target - one background run without muons incident on the target, and one with muons incident on the target. This included identifying photopeaks consistent with the background and identifying photopeaks unique to the muon run. I also calculated the minimum 
detectable activity for one of the muonic $\mathrm{x}$ rays detected.

I wrote a simulation based on the Bethe-Bloch equation to determine the dose equivalent deposited into a simplified human phantom for different path lengths and energy levels.

Preliminary work in this project was presented as a poster at the Canadian Radiation Protection Association annual conference. 


\section{Chapter 2}

\section{Related Work}

This chapter summarizes work in fields relating to this thesis, namely identifying special nuclear materials, muonic fission, dose rate of muons, and muonic x rays.

\subsection{Cosmic Ray Inspection and Passive Tomography (CRIPT)}

CRIPT is a project that similarly attempts to use muons to identify special nuclear material. However, whereas this thesis uses an accelerator to generate muons and examines the resulting muonic $\mathrm{x}$ rays, CRIPT uses cosmic ray muons and angular deflection for identification. CRIPT uses charged particle tracking detectors to measure the momentum and trajectory of incoming muons, and thus identify if high-Z materials are present in the target.

One of the key advantages of CRIPT over active muon interrogation is that CRIPT does not need an accelerator. However, CRIPT can neither identify the uranium isotope directly nor quantify the amount of the isotope, two things of which active muon interrogation is capable.

CRIPT relies on muon tomography, which is based on measuring multiple scat- 
tering of muons passing through high $Z$ materials [22]. Two tracking stations are used, one above and one below the object of interest. Both of them measure the track produced by cosmic muons, and the combined information is used to measure the deflection of the incident muon. The system can also measure the momentum of the $\mu^{ \pm}$as they pass through Fe slabs. This method relies strongly on algorithmic methods to reconstruct an image of the object of interest.

\subsection{Muon Induced Fission in Uranium}

Salahuddin Ahmad analyzed muon induced fission of uranium [21]. In his work, Ahmad examined the different methods of muon induced fission in both ${ }^{238} \mathrm{U}$ and ${ }^{235} \mathrm{U}$. He calculated the absolute yield for both of these isotopes, as well as the fission lifetimes. Ahmad distinguished between two types of muon induced fission, nonradiative transition (prompt) and nuclear capture (delayed). Ahmad's discussion of fission provided the basis of muon induced fission found in section 1.10.

\subsection{Radiation Weighting Factor for Slow Negative Muons}

The radiation weighting factor, $w_{R}$, is a number used to describe how damaging a particular form of radiation can be. Since the equivalent dose from muons is of interest to this thesis (as people could be in the way of the muon beam in a practical application), the weighting factor for muons needs to be calculated to properly calculate the equivalent dose. For example, the dose caused by an $\alpha$ particle at a given energy is 20 times more damaging than the same dose caused by an electron or photon [23].

In previous work on the dose from muons, Siiskonen used an MCNPX simulation to demonstrate that a radiation weighting factor of 1 underestimates the skin equivalent 
dose, whereas a weight of unity may be appropriate for organs [24]. Thus, when calculating the equivalent dose from muons, a weighting factor of $w_{R}=1$ has been used.

Siiskonen used a standard ICRU (International Commission on Radiation Units) sphere phantom consisting of hydrogen, carbon, nitrogen, and oxygen within an MCNPX Monte Carlo simulation. He calculated the weighting factors at a depth of $10 \mathrm{~mm}$ and at the location of the maximum weighting factor. He did this for both $1 \mathrm{MeV}$ and $10 \mathrm{MeV}$ muons. For the $1 \mathrm{MeV}$ muons, the maximum weighting factor occurs in the first $2 \mathrm{~mm}$, with a value of 3.14 to 8.60 depending on the thickness of the scoring region. For $10 \mathrm{MeV}$ muons, the maximum weighting factor occurs approximately $6 \mathrm{~mm}$ to $8 \mathrm{~mm}$ into the target, with a value of 1.94 .

\subsection{Measurement of Muonic Hyperfine Transition Rates and Muon Capture Yields in Light Nuclei}

Measurement of Muonic Hyperfine Transition Rates and Muon Capture Yields in Light Nuclei is Trevor Stocki's Ph. D thesis [25]. Stocki's thesis reports several different measurements involving muons. The experimental setup for $\gamma$ ray spectrometry was used as the basis for the setup that collected the data used in this thesis.

In 1998, Trevor Stocki published measurements of muonic hyperfine transition rates in light nuclei, specifically $\mathrm{LiF},\left(\mathrm{CF}_{2}\right)_{\mathrm{n}}, \mathrm{Na}, \mathrm{NaH}, \mathrm{Al}, \mathrm{LiAlH}_{4}$ [25]. The muonic hyperfine effect occurs as a result of nuclear spin. If a nucleus has a non-zero spin, then the energy levels of the orbital electrons (and muons) will split into two. Depending on which of the hyperfine states the muon populates, it can then undergo a transition to the other hyperfine state. This is known as the hyperfine transition. Stocki measured 
these transition rates in the nuclei mentioned above [26].

Two different experimental setups were used in Stocki's thesis. The first was a neutron detector. This consisted of four cylindrical liquid scintillators. These scintillators were used to discriminate between neutron and $\gamma$ events. They could do so through the use of pulse shape discrimination. This is because of the different way that neutrons and $\gamma$ rays interact. The second experimental setup was a high purity germanium (HPGe) gamma ray detector. The gamma ray setup used in Stocki's thesis was used as the basis of the experimental setup discussed later in this thesis. It consisted of two HPGe detectors, surrounding compton supressors, and electron veto scintillators.

A second purpose of Stocki's thesis (using the HPGe setup mentioned above) was to examine muonic x-rays and gamma rays from ${ }^{14} \mathrm{~N}$ using the HPGe gamma ray detector. Stocki examined the yields of several $\gamma$ rays from ${ }^{14} \mathrm{C},{ }^{13} \mathrm{C},{ }^{12} \mathrm{C}$, and ${ }^{10} \mathrm{~B}$, as well as the muonic $\mathrm{x}$ rays of ${ }^{14} \mathrm{~N}[27]$. 


\section{Chapter 3}

\section{Previous Work on the Project}

This chapter summarizes the experimental work of previous group members, as well as some initial analysis done by Jason Olsthoorn. Olsthoorn's calculations of acceptance as seen in section 3.3.1 are used as the accepted values when examining the EGSnrc simulation of acceptance.

\subsection{Muon Beam Production}

The muons for the experiment of Stocki et. al. [1] were produced at the TRIUMF National Lab. First, negatively charged hydrogen ions are accelerated in a cyclotron. This is done by using a mixture of electric fields to accelerate the ions and magnetic fields to keep the ions traveling in a circular path. Then, when the ions have reached an appropriate energy, a carbon foil is placed at the appropriate radius in the path of the beam to strip the electrons. The now positively charged protons change direction (as the force produced by the magnetic field changes direction) and exit the cyclotron down the beamline of interest.

The TRIUMF beamlines have energies ranging from $70 \mathrm{MeV}$ to $500 \mathrm{MeV}$, with a beam power ranging from $50 \mathrm{~kW}$ to $75 \mathrm{~kW}$ with a maximum current of $250 \mu \mathrm{A}$ [28]. Beamline 1A (BL1A) serves protons primarily to experimental channels, including 
detector tests for the T2K (Tokai to Kamioka) project, and $\mu \mathrm{SR}$ experimental channels. Secondary particles are also produced at targets along BL1A and sent down the different channels for pion or muon research. Beamline 1B (BL1B) is primarily used for the Proton Irradiation Facility (PIF) that is used for radiation testing of electronic devices. Beamline 2A (BL2A) provides proton beams for ISAC (Isotope Separation and ACceleration) and ISAC-II. The beam hits a target, creating certain isotopes which are mass selected into a new radioactive beam. Beamline 2C (BL2C) is used for proton therapy treatment for eye cancers. Beamline 4 (BL4) has not been used as a production facility since 2000. It is currently being extended into BL4N (North) for use with ISAC. It is also going to be used with the eLINAC being built at TRIUMF [29]. The TRIUMF facilities can be seen in Figure 3.1.

For this experiment, the BL1A beamline and the M20 channel were used. For muon production on the M20 channel, the proton beam strikes the target. The T2 target (see Figure 3.1) is typically a $100 \mathrm{~mm}$ thick piece of Be in a water cooling jacket [28]. After hitting the target, pions are produced and travel down the channel, where they decay into muons. Quadrupole magnets are used for focusing the beam, and dipole magnets are used for bending the muons as well as momentum selection.

\subsubsection{The Beam Telescope}

After exiting the focusing magnets, the beam of muons passes into the collimator. This only allows muons traveling in the center of the beam to pass through to the target, i.e. it removes any divergent muons from the beam. The collimator is made of lead bricks, and surrounded by polyethylene. If a muon were to stop in the lead, a great number of neutrons would be produced through nuclear capture of the muon, and these neutrons could damage the HPGe detector. The polyethylene prevents this from happening, as the rate of capture is proportional to $Z$. Instead, the muon will decay in orbit, creating an electron instead. The lead then stops these electrons. 


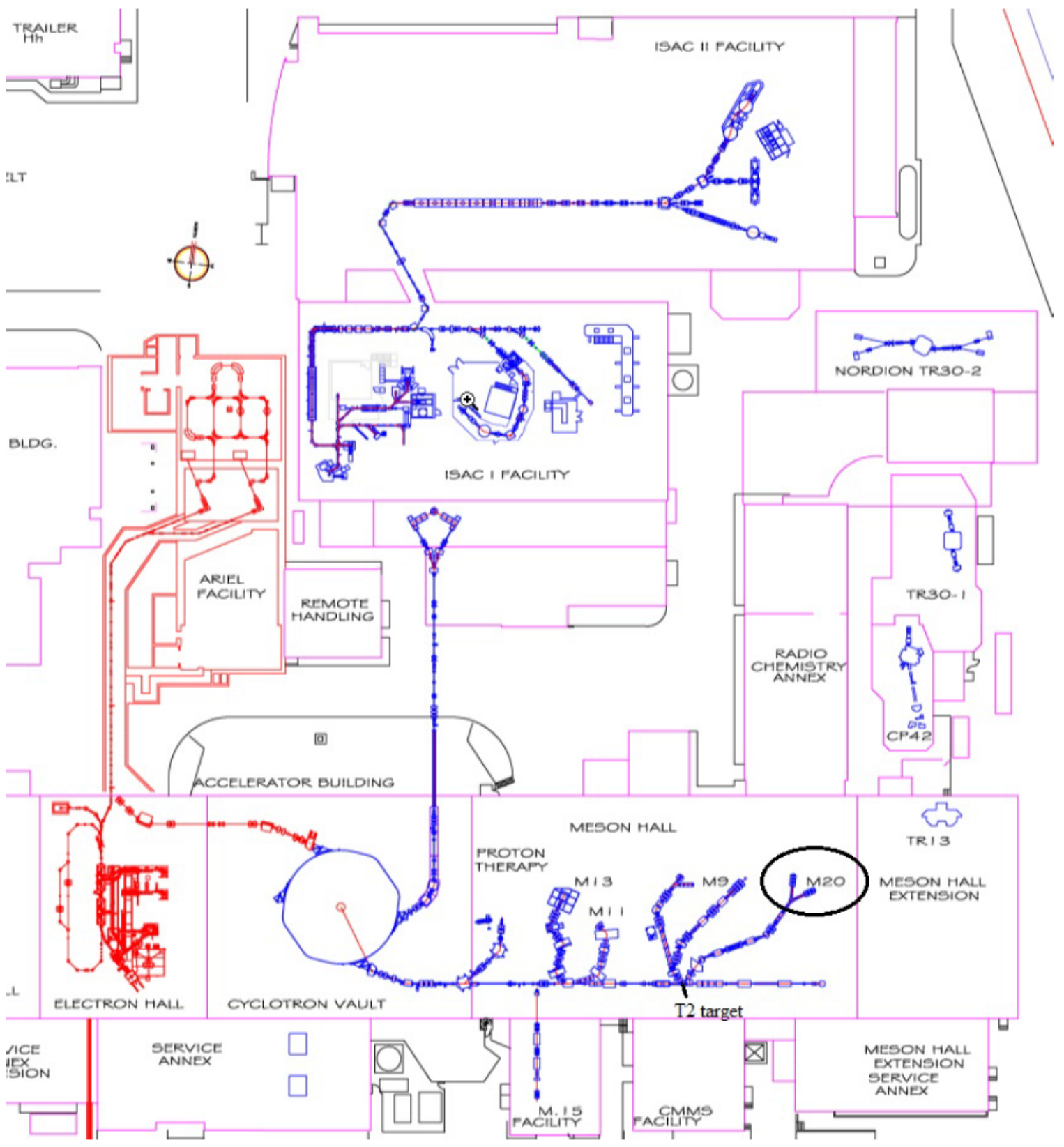

Figure 3.1: The various beamlines at TRIUMF. The M20 channel is circled, and the T2 target identified. Permission granted from Marcello Pavan [30]. 
After passing through the collimator, the beam passes through two scintillators, $\mathrm{S} 1(2 \mathrm{~cm}$ by $2 \mathrm{~cm})$ and $\mathrm{S} 2(5 \mathrm{~cm}$ by $5 \mathrm{~cm})$. A third scintillator was not used, so the number of muon stops was not directly known, but had to be calculated instead. This was done so as to simulate the most likely use case of attempting to detect SNM. After passing through the scintillators, the muon stops in the target. Several targets were used, including gold, uranium, and lithium chloride.

\subsection{Gamma Ray Experiment}

The experiment was performed by Stocki, Jason, et. al. [1]. A single GEM 140P4ST High Purity Germanium (HPGe) detector (produced by ORTEC Products Group, Oak Ridge, TN) was used for this experiment. It was surrounded by a BGO (bismuth germanium oxide) Compton suppressor. This was used to detect $\gamma$ rays (produced through the Compton effect) escaping the detector before depositing all of their energy. The suppressor rejected any events associated with a Compton scattering. A plastic scintillator was placed in front of the detector to act as a charged particle veto. The layout of the detector is given in Figure 3.2

\subsubsection{Sources and Targets Used}

The sources and targets used throughout the experiment are listed in Table 3.1. The calibration sources were used to calibrate both energy and acceptance for the detector. The multigamma sources such as ${ }^{133} \mathrm{Ba}$ and ${ }^{152} \mathrm{Eu}$ were kept far enough away from the detector so that coincidence summing (simultaneous incidence of two or more different gamma rays on the detector, causing a peak at higher energies) was not a concern. Other target configurations were also used to determine if it is possible to hide the uranium, but are not the subject of this thesis. 


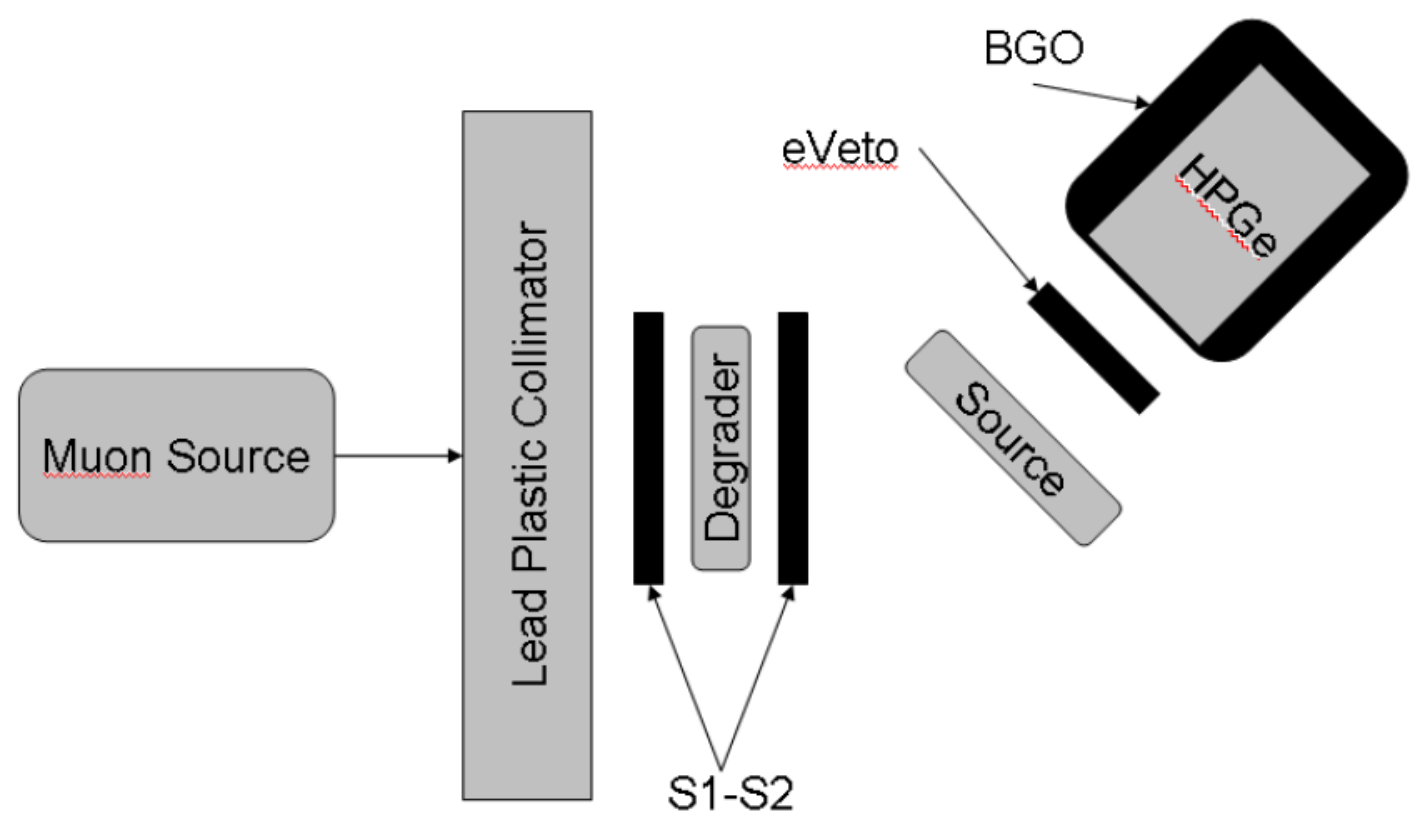

Figure 3.2: Diagram of the experimental setup [1]. Image used with permission of T. Stocki.

Table 3.1: Some of the various sources and targets used during the experiment. Others were used, but only runs involving these materials were analyzed for this thesis.

\begin{tabular}{|l|l|}
\hline Source/Target & Purpose \\
\hline${ }^{22} \mathrm{Na}$ & Calibration \\
${ }^{60} \mathrm{Co}$ & Calibration \\
${ }^{133} \mathrm{Ba}$ & Calibration \\
${ }^{137} \mathrm{Cs}$ & Calibration \\
${ }^{152} \mathrm{Eu}$ & Calibration \\
$\mu^{-85} \mathrm{Au}$ & Calibration \\
${ }^{238} \mathrm{U}$ & Target \\
${ }^{n a t} \mathrm{Cl}$ & Target \\
\hline
\end{tabular}




\subsubsection{Electronics}

The electronics setup is shown in Figure 3.3. The thresholds on the S1 and S2 discriminators were set to be above any electrons related to the beam. A coincidence was formed between them to ensure the beam was on target. A 40 ps gate was formed from the S1·S2 coincidence, which was then placed into coincidence with the HPGe detector to form a master gate. This was used to start a VME-based data-acquisition system. The signals from both the HPGe and the Compton suppressor were sent through a timing filter amplifier to improve the timing, and then sent into a constant fraction discriminator (CFD). The HPGe signal was also sent into an analog to digital converter (ADC). The various time signals were all sent to a multi-hit time to digital converter (TDC). 


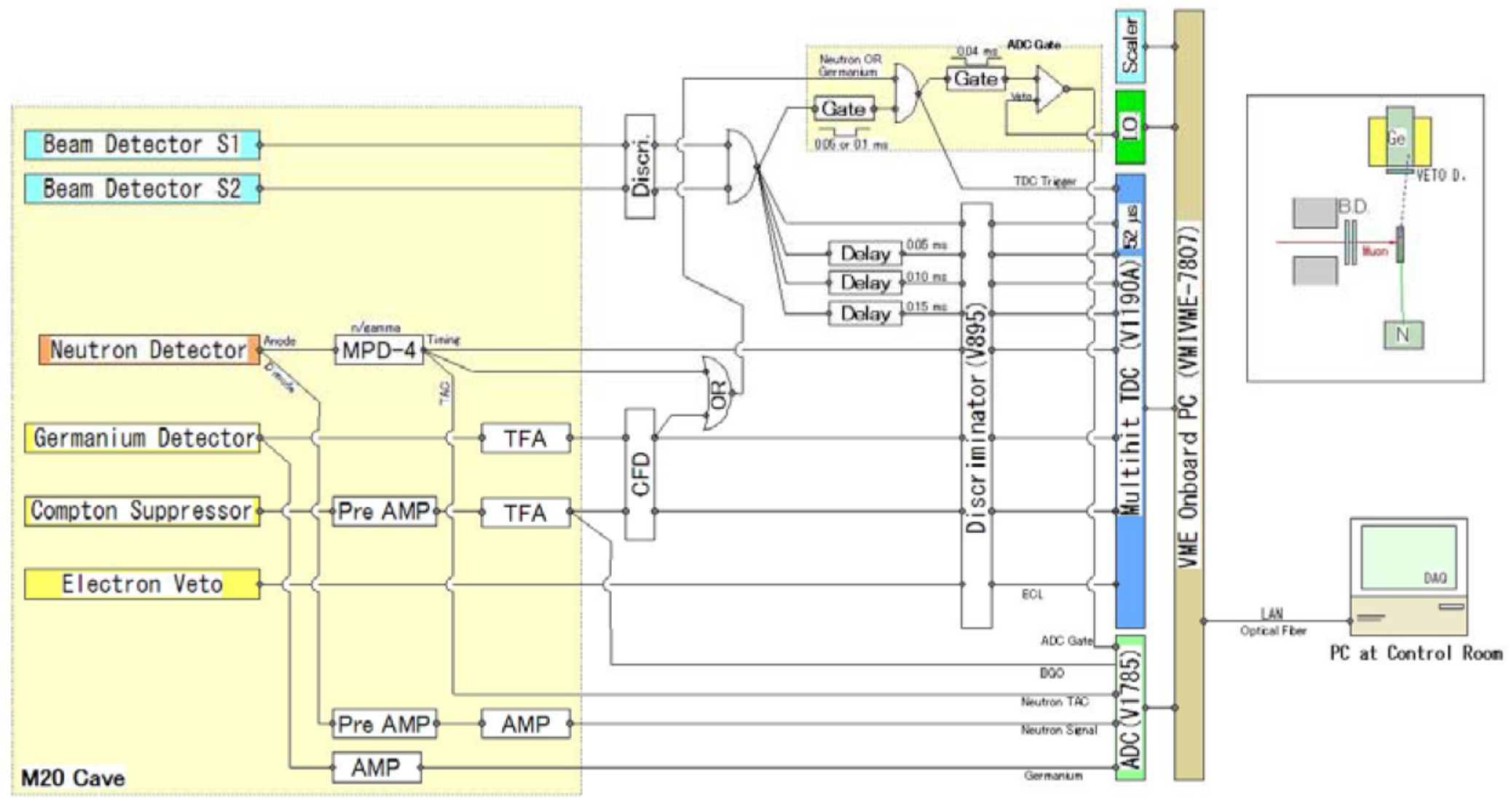

Figure 3.3: Schematics for the electronics for the experiment [1]. 


\subsubsection{Data Acquisition}

The data were acquired through a PC running Linux. ROOT, a software package by CERN [31], was used for data collection and analysis. The data were originally saved in binary files, and converted to .root with a program written by Andrew Green, one of the group members who assisted with the experiment.

\subsection{Muon Interaction with Natural Chlorine}

Previous work has been performed by Jason Olsthoorn on data obtained from the experiment used in this thesis [4]. Olsthoorn used several radioactive sources to calibrate the energy, as seen in Table 3.2. Olsthoorn's calibration was used as the starting point for the energy calibration later in this thesis.

Table 3.2: Energies used for energy calibration by Jason Olsthoorn [4].

\begin{tabular}{|c|c|}
\hline Origin & Energy $(\mathrm{keV})$ \\
\hline Annihilation & 511.0 \\
${ }^{137} \mathrm{Cs}$ & 661.66 \\
${ }^{41} \mathrm{Ar}$ & 1293.64 \\
${ }^{60} \mathrm{Co}$ & 1332.49 \\
${ }^{34} \mathrm{~S}$ & 2127.49 \\
$\mathrm{H}(n, \gamma)$ & 2223.25 \\
${ }^{208} \mathrm{~Pb}\left(n, n^{\prime} \gamma\right)$ & 2614.51 \\
${ }^{196} \mathrm{Pt} \gamma$ & 5255.30 \\
$\mathrm{Fe}(n, \gamma)$ & 7631.18 \\
$\mathrm{Fe}(n, \gamma)$ & 7645.58 \\
\hline
\end{tabular}

\subsubsection{Acceptances}

The acceptance (also known as absolute full energy peak efficiency) is the ratio of the number of counts detected to the number emitted [19]. This depends on the geometry of the source (or target) and detector. By knowing the acceptance, one can calculate the number of counts one would expect for a different target placed in 
the same arrangement. For this thesis, acceptance needed to be calculated for two different sources of photons: radioactive sources and muonic $\mathrm{x}$ rays.

For a radioactive source, acceptance can be calculated using equation (3.1) [25], where $\xi$ is the acceptance, $A$ is the number of counts in the peak, $f_{s a}$ is the selfabsorption coefficient (which accounts for the number of photons produced that never exit the material), $R$ is the real time in seconds, $L$ is the live time proportion of $R$, $A_{c}$ is the activity, and $E_{p}$ is the emission probability of the $\gamma$ ray. The self-absorption coefficient was calculated using equation (3.2), where $\mu$ is the mass attenuation coefficient at the gamma energy, $\rho$ is the density of the element, and $X$ is the half-thickness of the source. Acceptances for muonic $\mathrm{x}$ ray emissions are calculated using a similar equation, equation (3.3), where $S$ is the number of muon stops in the target, and $E_{p}$ is the emission probability of the $\mu^{-} \mathrm{x}$ ray.

$$
\begin{gathered}
\xi_{\text {radioactive }}=\frac{A f_{s a}}{R L A_{c} E_{p}} \\
f_{s a}=\exp (\mu \rho X) \\
\xi_{\text {muon }}=\frac{A f_{s a}}{S L E_{p}}
\end{gathered}
$$

In order to determine the efficiency of the detector, an acceptance calibration was performed at various known energies in order to provide an efficiency curve. Acceptances were calculated for two cases. Radioactive sources were used for acceptances for energies less than $2 \mathrm{MeV}$, and muonic $\mathrm{x}$ rays were used energies greater than $2 \mathrm{MeV}$. The muon sources were used to calibrate acceptance at the higher energy range, as the radioactive sources do not emit $\gamma$ rays at these energies. For the radioactive sources, acceptances were calculated using equation (3.1). The self-absorption coefficient was calculated using equation (3.2). The emission probabilities for the muonic $\mathrm{x}$ rays were taken from Hartmann et al [32]. 
Table 3.3: The measured acceptances as calculated by Olsthoorn [4].

\begin{tabular}{|c|l|l|}
\hline Energy (keV) & Source & Acceptance \\
\hline 121.7817 & Eu152 & $(3.89 \pm 0.04) \times 10^{-3}$ \\
244.6875 & Eu152 & $(2.74 \pm 0.03) \times 10^{-3}$ \\
276.3997 & Ba133 & $(2.46 \pm 0.03) \times 10^{-3}$ \\
302.851 & Ba133 & $(2.356 \pm 0.019) \times 10^{-3}$ \\
344.2785 & Eu152 & $(2.354 \pm 0.015) \times 10^{-3}$ \\
356.0134 & Ba133 & $(2.209 \pm 0.009) \times 10^{-3}$ \\
383.848 & Ba133 & $(2.112 \pm 0.018) \times 10^{-3}$ \\
411.1163 & Eu152 & $(2.06 \pm 0.04) \times 10^{-3}$ \\
443.965 & Eu152 & $(2.07 \pm 0.04) \times 10^{-3}$ \\
778.904 & Eu152 & $(1.583 \pm 0.015) \times 10^{-3}$ \\
867.373 & Eu152 & $(1.475 \pm 0.021) \times 10^{-3}$ \\
964.079 & Eu152 & $(1.430 \pm 0.011) \times 10^{-3}$ \\
1112.069 & Eu152 & $(1.36 \pm 0.06) \times 10^{-3}$ \\
1173.228 & Co60 & $(1.370 \pm 0.014) \times 10^{-3}$ \\
1212.948 & Eu152 & $(1.29 \pm 0.04) \times 10^{-3}$ \\
1274.537 & Na22 & $(1.214 \pm 0.010) \times 10^{-3}$ \\
1299.142 & Eu152 & $(1.13 \pm 0.03) \times 10^{-3}$ \\
1332.492 & Co60 & $(1.244 \pm 0.013) \times 10^{-3}$ \\
1408.006 & Eu152 & $(1.185 \pm 0.009) \times 10^{-3}$ \\
2341.2 & $\mu^{-}$Au & $(7.5 \pm 1.3) \times 10^{-4}$ \\
2477.8 & $\mu^{-}$Au & $(7.3 \pm 1.5) \times 10^{-4}$ \\
5591 & $\mu^{-}$Au & $(4.3 \pm 0.8) \times 10^{-4}$ \\
5763.1 & $\mu^{-}$Au & $(4.3 \pm 1.5) \times 10^{-4}$ \\
\hline
\end{tabular}

For the muon sources, $\mu^{-} \mathrm{Au} \mathrm{x}$ rays were used. Acceptances for these $\mathrm{x}$ ray emissions were calculated using a similar equation, equation (3.3). In a typical muon beam telescope, three colinear scintillators are used, two ( $S 1$ and $S 2)$ to verify that the particle will enter the target, and a third $(S 3)$ to detect if the particle passed through without stopping. No $S 3$ scintillator was present on the far side of the target, so muon stops in gold were determined through the use of acceptance estimation for the $\mu^{-} \mathrm{Au} \mathrm{x}$ rays at energies of $400.15 \mathrm{keV}$ and $405.58 \mathrm{keV}$, which are within the range of radioactive source energies. These calculations are used as the accepted values for the purposes of comparing the simulation against the known values, and can be seen in Table 3.3. 


\subsubsection{Chlorine Spectrum Analysis}

Olsthoorn [4] analyzed a muon beam run with natural chlorine as the target. $\mathrm{He}$ identified several muonic $\mathrm{x}$ rays for chlorine. He also identified $\gamma$ rays from various isotope of sulfur. The sulfur itself was produced as a result of muonic nuclear capture. He also calculated the lifetime of a muon in the $1 s$ position in chlorine. The methods Olsthoorn used for analyzing the chlorine spectrum were used as the basis for the methods used in this thesis. 


\section{Chapter 4}

\section{Data Analysis}

\subsection{Energy Calibration}

The data obtained from the HPGe detector are the number of counts in each channel. However, the energy corresponding to each channel is not known. Thus, a function describing the energy for a given channel is necessary. This energy calibration allows for a proper analysis to be done, as without it, only the channel numbers are known.

Two different runs were used for energy calibration. In the first run, data obtained using a ${ }^{133} \mathrm{Ba}$ source were used. In the second run, data from ${ }^{60} \mathrm{Co}$ were used. For calibration at the higher energies, ${ }^{1} \mathrm{H},{ }^{57} \mathrm{Fe}$, and ${ }^{208} \mathrm{~Pb}(\mathrm{n}, \gamma) \gamma$ rays were used. These are self-calibrating background lines. Self-calibrating lines are lines with wellknown energies that one expects to see in the background. The data were then fit to equation (4.1) (an empirical formula) [33], where $E$ is the energy, $a_{n}$ are the fitting parameters, and $x$ is the channel number.

$$
E=a_{0} x^{2}+a_{1} x+a_{2}
$$




\subsubsection{FitzPeaks Gamma Analysis Software}

FitzPeaks Gamma Analysis and Calibration Software is a program developed by Jim Fitzgerald [34]. It was thought that FitzPeaks could aid in the detection of peaks, as it has several modern algorithms to search for and identify various peaks. Although it did do a fair job of identifying obvious peaks, it had trouble identifying peaks in the higher energy region. A manual approach to identifying the peaks proved to be more fruitful, and was the sole approach used in this thesis. It is described below in section 4.2 .

In order to import the data into FitzPeaks, the ROOT histogram data were exported to a $\mathrm{C}$ file format. A script was then written to format the data so that FitzPeaks would accept it. FitzPeaks specifically looked for 8 blank lines at the start (which can be used for various metadata), followed by width delimited columns of 8 channels per line.

\subsection{Peak Fitting}

After deciding not to use FitzPeaks as a method of peak analysis, peak fitting was instead done using the ROOT statistics package developed by CERN (European Organization for Nuclear Research) [31]. Functions used were of the forms seen in equations (4.2) and (4.3) [35], where $a_{n}$ is the $n$-th fitting parameter, and erfc is the complimentary error function. Equation (4.2) is a gaussian shape with an complimentary error function background. The complimentary error function is used as the background to account for the fact that Compton effects will only be seen on the lower end of the background, and because the error function accounts for the resolution of the detector. Equation (4.3) is a double gaussian with a quadratic background. Equation (4.3) was used when two peaks were too close to separate and leave enough 
degrees of freedom for fitting.

$$
\begin{gathered}
f(x)=a_{0} \exp \left(\frac{\left(x-a_{1}\right)^{2}}{2 a_{2}^{2}}\right)+\frac{1}{2} a_{3} \operatorname{erfc}\left(\frac{x-a_{1}}{\sqrt{2} a_{2}}\right)+a_{4} \\
f(x)=a_{0} \exp \left(\frac{\left(x-a_{1}\right)^{2}}{2 a_{2}^{2}}\right)+a_{3} \exp \left(\frac{\left(x-a_{4}\right)^{2}}{2 a_{5}^{2}}\right)+a_{6} x^{2}+a_{7} x+a_{8}
\end{gathered}
$$

The fitting itself was done by manually scanning through the spectrum, and when a potential peak was found, the peak was fit within a given energy. A peak was accepted only if the reduced $\chi^{2}$ value was approximately 1 , indicating a good fit and avoiding both over-fitting and poor fits. This led some apparent peaks to be discarded as the peak was a poor fit.

\section{$4.3 \quad \mu^{-} \mathrm{U}$ X Rays}

Two runs were analyzed for the purpose of identifying muonic $\mathrm{x}$ rays and $\gamma$ rays after muon capture. One run had been taken without muons incident on the target, and another with muons incident on the target. Photon peaks were identified the equations found in section 4.2. The peaks identified on the beam-off run were then assumed to be background radiation, and any of these background peaks that were identified in the beam on run were not considered to be beam related.

\subsubsection{Background Peaks}

The background peaks fall into one of several categories:

Ambient background photons These are peaks caused by the ambient radiation in the background at the location of the detector. Examples of these gamma rays include the annihilation peak, ${ }^{40} \mathrm{~K}$ gamma rays, or ${ }^{60} \mathrm{Co}$ lines. 
- Some lines, such as ${ }^{60} \mathrm{Co}$ or ${ }^{137} \mathrm{Cs}$ are not normal ambient background photons, but could be seen because these sources are present in the experimental area. It is also possible that the ${ }^{60} \mathrm{Co}$ is present from the activation of the stainless steel in the beam pipe.

Uranium decay $\gamma$ rays These are peaks that are caused from the decay of ${ }^{238} \mathrm{U}$. These are gamma rays caused from nuclear de-excitation after $\alpha$ or $\beta$ decay of the isotopes in the ${ }^{238} \mathrm{U}$ decay chain (see Figure 6.1). These include various isotopes such as ${ }^{230} \mathrm{Th},{ }^{214} \mathrm{Bi},{ }^{234} \mathrm{~Pa}$, and others.

Neutron related peaks These are $\gamma$ rays that are caused by the interaction of neutrons with various elements in the detector and nearby housing, as discussed in section 1.8.1. This includes peaks like the ${ }^{27} \mathrm{Al}(n, \gamma) \gamma$ ray, and ${ }^{73} \mathrm{Ge}$ or ${ }^{72} \mathrm{Ge}$ $(n, \gamma)$ gamma rays.

The background peaks are listed in Table 4.1. There are some issues with the identification of some of the lines. For example, the ${ }^{234} \mathrm{U}$ peak at $53.2 \mathrm{keV}$ should indicate the presence of a peak at $120.90 \mathrm{keV}$, which was not seen in the background run. Similarly, for the $77.107 \mathrm{keV}$ peak, there should also peaks at $295.2228 \mathrm{keV}$ and $351.9321 \mathrm{keV}$, which again are not detected in the background run.

\subsubsection{Fission Products}

There are three types of fission possible, as discussed in section 1.10. The first is spontaneous fission, where the nucleus splits into two smaller nuclei on its own. The second is induced by energy from the atomic cascade of the muon. In this case, the nucleus absorbs energy from the atomic transition of the muon, bringing it into an excited state and causing fission. The third and final type is caused by nuclear muon capture. In this scenario, the muon changes a proton within the nucleus into a neutron, and leaves the nucleus in an excited state, which can cause fission [17]. 
Table 4.1: Background peaks identified in both uranium runs, with and without muons incident. Number of counts is the number in the second run, with the muon beam incident on the target. The $\dagger$ indicates a lack of confidence in the identification. These peaks should correspond with other peaks that were not detected.

\begin{tabular}{|r|c|c|c|}
\hline Energy $(\mathrm{keV})$ & Expected Energy $(\mathrm{keV})$ & Identification & Number of Counts \\
\hline $53.1 \pm 0.2$ & 53.2 & ${ }^{234} \mathrm{U} \dagger$ & $(1.078 \pm 0.017) \times 10^{5}$ \\
$77.6 \pm 0.2$ & 77.107 & ${ }^{214} \mathrm{~Pb} \mathrm{~K} \alpha 1 \dagger$ & $(7.60 \pm 0.13) \times 10^{4}$ \\
$85.8 \pm 0.2$ & 85.431 & ${ }^{230} \mathrm{Th} \mathrm{K} \alpha 2$ & $(5.14 \pm 0.10) \times 10^{5}$ \\
$511.3 \pm 0.2$ & 511 & Annih & $(1.134 \pm 0.012) \times 10^{5}$ \\
$585.5 \pm 1.5$ & 510.77 & ${ }^{208} \mathrm{Tl}$ & \\
$610.0 \pm 1.4$ & 583 & ${ }^{208} \mathrm{Tl}$ & $(1.8 \pm 1.0) \times 10^{3}$ \\
$662.2 \pm 0.3$ & 609.32 & ${ }^{214} \mathrm{Bi}$ & $(1 \pm 8) \times 10^{3}$ \\
$898.3 \pm 0.4$ & 661.657 & ${ }^{137} \mathrm{Cs}$ & $(3.4 \pm 0.4) \times 10^{3}$ \\
$1001.1 \pm 0.3$ & 1001.03 & & $(1.5 \pm 0.2) \times 10^{3}$ \\
$1041.9 \pm 0.8$ & 1041.7 & ${ }^{234 m} \mathrm{~Pa}$ & $(1.662 \pm 0.007) \times 10^{5}$ \\
$1202.5 \pm 0.3$ & 1202.58 & ${ }^{234 m} \mathrm{~Pa}$ & $(8 \pm 3) \times 10^{2}$ \\
$1293.4 \pm 0.4$ & 1293.64 & ${ }^{206} \mathrm{Bi}$ & $(8.5 \pm 0.2) \times 10^{3}$ \\
$1332.6 \pm 0.4$ & 1332.492 & ${ }^{41} \mathrm{Ar}$ & $(7.6 \pm 0.2) \times 10^{3}$ \\
$1377.8 \pm 0.5$ & 1377.669 & ${ }^{60} \mathrm{Co}$ & $(1.89 \pm 0.18) \times 10^{3}$ \\
$1460.9 \pm 0.4$ & 1460.882 & ${ }^{214} \mathrm{Bi}$ & $(3 \pm 10) \times 10^{2}$ \\
$1509.9 \pm 0.4$ & 1509.21 & ${ }^{40} \mathrm{~K}$ & $(3.03 \pm 0.15) \times 10^{3}$ \\
$1765.0 \pm 0.4$ & 1765.44 & ${ }^{214} \mathrm{Bi}$ & $(2.41 \pm 0.14) \times 10^{3}$ \\
& 1764.491 & ${ }^{234} \mathrm{~Pa}$ & $(1.91 \pm 0.17) \times 10^{3}$ \\
$1778.7 \pm 0.6$ & 1779.03 & ${ }^{214} \mathrm{Bi}$ & \\
$1809.0 \pm 0.5$ & 1809.04 & ${ }^{27} \mathrm{Al}(n, \gamma)$ & $(4.2 \pm 1.2) \times 10^{2}$ \\
$1831.0 \pm 0.5$ & 1831.36 & ${ }^{234} \mathrm{~Pa}$ & $(8.7 \pm 1.2) \times 10^{2}$ \\
$2222.7 \pm 0.6$ & 2223.245 & ${ }^{234} \mathrm{~Pa}$ & $(2.80 \pm 0.15) \times 10^{3}$ \\
$2614.4 \pm 0.6$ & 2614.511 & ${ }^{2} \mathrm{H}(n, \gamma)$ & $(4.7 \pm 1.5) \times 10^{2}$ \\
$3536 \pm 4$ & 3538.16 & ${ }^{208} \mathrm{Tl}$ & $(9.4 \pm 1.0) \times 10^{2}$ \\
$4493.3 \pm 1.7$ & 4492 & $\mathrm{Ge}(n, \gamma)$ & $(2 \pm 4) \times 10^{1}$ \\
$6715.2 \pm 1.9$ & 6715.2 & $\mathrm{Ge}(n, \gamma)$ & $(6 \pm 5) \times 10^{1}$ \\
& & & $(6 \pm 3) \times 10^{1}$ \\
\hline
\end{tabular}


Since it is possible for uranium to undergo fission, it is also possible that the uranium undergoes fission while the muon is captured on the nucleus. In that case, it is possible that muonic $\mathrm{x}$ rays are produced while interacting with the fission products.

The fissile yields are listed in Table 4.2. The fissile yield is defined as the probability of obtaining the given element for a fission event. The yields are normalized so that the total yield of ${ }^{238} \mathrm{U}$ is unity.

Table 4.2: The yield (percentage of fission products that result in that element) of fission products of ${ }^{238} \mathrm{U}$ [36]. Uncertainties were not available. The yields are normalized so that the total yield of ${ }^{238} \mathrm{U}$ is unity.]

\begin{tabular}{|c|c||c|c|}
\hline$Z$ & Yield & $Z$ & Yield \\
\hline 36 & $4.60 \times 10^{-3}$ & $\mathbf{5 3}$ & $8.32 \times 10^{-2}$ \\
$\mathbf{4 2}$ & $5.99 \times 10^{-2}$ & 54 & $2.04 \times 10^{-1}$ \\
43 & $7.28 \times 10^{-2}$ & $\mathbf{5 5}$ & $1.52 \times 10^{-1}$ \\
44 & $1.75 \times 10^{-1}$ & $\mathbf{6 0}$ & $9.91 \times 10^{-2}$ \\
$\mathbf{4 5}$ & $4.77 \times 10^{-2}$ & 61 & $4.93 \times 10^{-2}$ \\
$\mathbf{4 6}$ & $2.39 \times 10^{-2}$ & 62 & $1.55 \times 10^{-2}$ \\
47 & $2.94 \times 10^{-3}$ & 63 & $6.49 \times 10^{-3}$ \\
48 & $5.30 \times 10^{-4}$ & 64 & $1.59 \times 10^{-3}$ \\
49 & $4.17 \times 10^{-4}$ & 66 & $2.19 \times 10^{-5}$ \\
51 & $5.66 \times 10^{-4}$ & 67 & $8.95 \times 10^{-7}$ \\
52 & $2.79 \times 10^{-4}$ & 68 & $3.05 \times 10^{-9}$ \\
\hline
\end{tabular}

The highest strength muonic $\mathrm{x}$ rays for the highest yield elements were searched for. The candidates for detection are listed in Table 4.3.

\subsubsection{Gamma Rays from Muonic Nuclear Capture}

As mentioned in section 3.3, it is possible to detect $\gamma$ rays emitted by atoms produced after nuclear capture of the muon. In Olsthoorn's report [4], he was able to identify several $\gamma$ rays from ${ }^{34} \mathrm{~S}$ and other sulfur isotopes. Since sulfur $(Z=16)$ was present because of nuclear muonic capture transforming a proton into a neutron within chlorine $(Z=17)$, it is likely that protactinium $(\mathrm{Pa}, Z=91)$ would be present from nuclear muonic capture on uranium $(Z=92)$. However, the level diagrams for the various isotopes of $\mathrm{Pa}$ (such as ${ }^{237} \mathrm{~Pa}$ or ${ }^{236} \mathrm{~Pa}$ for $1 n$ and $2 n$ emission events) are 
Table 4.3: Energies of the $2 p_{3 / 2} \rightarrow 1 s_{1 / 2}$ muonic x-ray transitions in various elements and isotopes [18]. Energies were not available for $Z=44,54$.

\begin{tabular}{|c|c|l|}
\hline$Z$ & Element & Energy (keV) \\
\hline 42 & ${ }^{92} \mathrm{Mo}$ & 2732.3 \\
42 & ${ }^{96} \mathrm{Mo}$ & 2713.3 \\
42 & ${ }^{98} \mathrm{Mo}$ & 2707.1 \\
45 & ${ }^{103} \mathrm{Rh}$ & 2982 \\
46 & ${ }^{n a t} \mathrm{Pd}$ & 3077 \\
53 & ${ }^{127} \mathrm{I}$ & 3721.6 \\
53 & ${ }^{127} \mathrm{I}$ & 3723.23 \\
55 & ${ }^{133} \mathrm{Cs}$ & 3899.1 \\
55 & ${ }^{133} \mathrm{Cs}$ & 3902.18 \\
60 & ${ }^{n a t} \mathrm{Nd}$ & 4335 \\
60 & ${ }^{142} \mathrm{Nd}$ & 4352.52 \\
60 & ${ }^{142} \mathrm{Nd}$ & 4352.06 \\
60 & ${ }^{144} \mathrm{Nd}$ & 4336.38 \\
60 & ${ }^{146} \mathrm{Nd}$ & 4321.18 \\
60 & ${ }^{148} \mathrm{Nd}$ & 4303.46 \\
60 & ${ }^{150} \mathrm{Nd}$ & 4266.37 \\
\hline
\end{tabular}

either not known or are classified. Thus, no analysis similar to Olsthoorn's could be done to identify these $\gamma$ rays. Only the difference between runs with and without muons incident on the target could be determined.

\subsection{EGSnrc simulation of acceptances}

To verify the acceptances calculated by Olsthoorn [4], Monte Carlo radiation transport simulations were run using EGSnrcMP V4-r2-4-0. The geometry for the EGSnrc simulation can be seen Figure 4.1. In previous work, the pre-built user-code DOSRZnrc had been modified by Lindsay Beaton into a program called energyBins [37]. The energyBins modification uses DOSRZnrc as the basis, but instead of using the default bin widths, it manually sets the widths to be more sensitive than DOSRZnrc allows for by default. This was also done to verify the acceptance simulation of others in the group who used MCNPX as the simulation toolkit.

The primary generator modeled a point source at the location of the target, emit- 
ting $\mathrm{x}$ and $\gamma$ rays of the appropriate energy. For the muonic $\mathrm{x}$ rays, the orbital capture was not simulated. EGSnrc only handles electron, positron, and photon interactions, and does not contain the relevant physics for orbital decay of muons. GEANT4 was examined to see if it had the relevant physics list available, and while it does handle several muon interactions (including decay, positron electron pair production, and photonuclear interaction), it does not provide the physics for muon orbital decay. Thus, EGSnrc was considered sufficient for modeling the photons produced after muonic orbital decay. The decay probabilities were taken from experimental results [38].

A sample .egsinp file used for simulating the acceptance of ${ }^{22} \mathrm{Na}$ (arbitrarily chosen) can be seen in Appendix A. The simulations lasted for $1.27 \times 10^{6}$ histories. For most of the options, the default is sufficient, but some need to be changed if the geometry is modified. Details can be found in Appendix A.

Each $\gamma$ or $\mathrm{x}$ ray was simulated separately, and the acceptances calculated from the DOSRZnrc output (.egslst file), as seen in equation (4.4), where $\varepsilon$ is the efficiency of the peak, $\Omega$ is the solid angle subtended by the detector housing (from the source point), and $f$ is the fraction of particles incident on the housing that caused a detector pulse. All of these were outputs taken from EGSnrc.

$$
\xi=\varepsilon \Omega f
$$

\subsection{Dose from Muons}

As mentioned in section 1.2, the equivalent dose deposited by muons in the human body is of interest. To calculate this, a multi-step procedure was used. First, the energy deposited by a muon was calculated, then the dose equivalent, and finally the upper limit on the number of muons was calculated. 


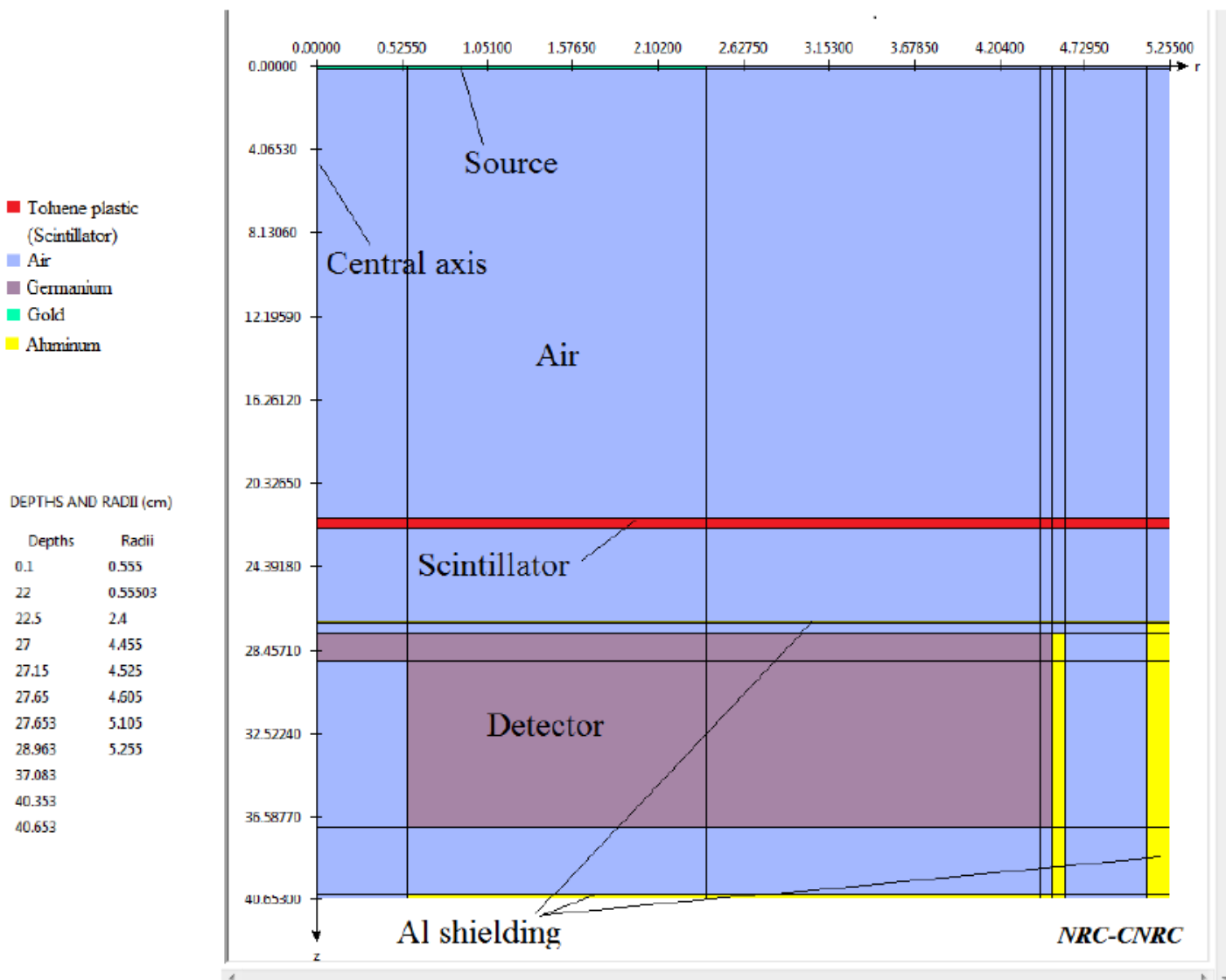

Figure 4.1: Geometry used in the acceptance simulation. The germanium detector is surrounded by air and aluminum. All distances are given in $\mathrm{cm}$. For the $\mu^{-} \mathrm{Au} \mathrm{x}$ rays, $0.1 \mathrm{~cm}$ of gold replaces the first $0.1 \mathrm{~cm}$ of air at the top.

In order to calculate the dose equivalent from one muon into the human body, the Bethe-Bloch equation for heavy particles was used to calculate the energy lost per distance traveled,

$$
\frac{\mathrm{d} E}{\mathrm{~d} x}=4 \pi r_{0}^{2} N_{e} \frac{m_{0} c^{2}}{\beta^{2}}\left[\ln \frac{2 m_{0} c^{2} \beta^{2}}{I\left(1-\beta^{2}\right)}-\beta^{2}-\frac{C(Z, \beta)}{Z}\right]
$$

where $c$ is the speed of light, $Z$ is the atomic number of the target material, $r_{0}=$ $2.818 \times 10^{-13} \mathrm{~cm}$ is the classical electron radius, $N_{e}$ is the electron density of the target in $\mathrm{g}^{-1}, m_{0}=511 \mathrm{keV} / \mathrm{c}^{2}$ is the electron rest mass, $I$ is the mean ionization potential of the target, $\beta$ is the velocity of the particle as a fraction of the speed of light, and $C(Z, \beta) / Z$ is a target dependent correction factor. An exact correction factor for the phantom was not available, and hence was taken to be 0 . However, given 
that the correction factors are always positive [39], it is the case that the stopping powers calculated will be upper limits, and hence any doses calculated will be upper limits as well.

For the purposes of simulation, a human phantom was modeled as a rectangular prism of dimensions $30 \mathrm{~cm}$ by $46 \mathrm{~cm}$ by $175 \mathrm{~cm}$, with a mass of $70 \mathrm{~kg}$. The phantom was composed of muscle, as defined in The Physics of Radiology by Johns and Cunningham [9], where all of the following constants are obtained. The mean ionization potential is given as $I=75.9 \mathrm{eV}$, and the electron density is given as $N_{e}=3.312 \times 10^{23} \mathrm{~g}^{-1}$. The density used was $\rho=1.04 \mathrm{~g} \mathrm{~cm}^{-3}$. Note that the actual density of the phantom according to its mass and dimensions would be $0.29 \mathrm{~g} \mathrm{~cm}^{-3}$. However, the dimensions were chosen primarily to simulate the maximum straight line distance through an average human being, and thus most of the phantom is actually empty.

A crude algorithm was used to calculate the dose deposited by a single muon.

1. Start with a given kinetic energy $T$, and path length $x$.

2. Calculate the stopping power, $S$, for the given energy, using equation 4.6.

$$
S=\frac{1}{\rho} \frac{\mathrm{d} E}{\mathrm{~d} x}
$$

3. Calculate the energy lost, $\mathrm{d} E=S \rho \mathrm{d} x$, where $\mathrm{d} x=x / 1000$

4. Recalculate $T^{\prime}=T-\mathrm{d} E$.

5. Continue until either $T=0$ or the particle has left the path length.

Once the energy deposited in the phantom by a single muon was calculated, the dose equivalent deposited in the phantom was calculated using equation (4.7), where $m=70 \mathrm{~kg}$, and $w_{R}=1$ is the radiation weighting factor for muons. A weighting factor of 1 was chosen because, while $w_{R}>1$ for skin dose, $w_{R}=1$ for anything below skin depth. Finally, the number of muon stops required to attain a dose equivalent of $1 \mathrm{mSv}$ was calculated using equation (4.8). 


$$
\begin{aligned}
& H=\frac{E_{\mathrm{dep}}}{m} \cdot W_{R} \\
& \text { stops }=\frac{1 \mathrm{mSv}}{H}
\end{aligned}
$$




\section{Chapter 5}

\section{Results}

This chapter summarizes the results of the analysis described in Chapter 4. It includes the energy calibration, the spectrum analysis, the results of the EGSnrc simulations, and the equivalent dose limits from muons.

\subsection{Energy Calibration}

Table 5.1 contains the specific energies used for calibration. The ${ }^{60} \mathrm{Co}$ run was used to get an initial energy calibration, as the two cobalt peaks are very clear to see. The calibration was then updated with the ${ }^{133} \mathrm{Ba}$ run, using the initial calibration to get an estimate of where the barium peaks should be seen. The calibration was finalized with the $(n, \gamma)$ events seen in the ${ }^{60} \mathrm{Co}$ run to handle any non-linearities in the upper portion $(E>1 \mathrm{MeV})$ of the energy spectrum. As mentioned in section 4.1, ${ }^{152} \mathrm{Eu}$ was not used for calibration, as it did not add any value to the area of interest $(E>2 \mathrm{MeV})$. Table 5.2 contains the calibration parameters used in equation (4.1). 
Table 5.1: Sources used for energy calibration, their energies, and respective channels [40]. $\varepsilon$ is electron capture.

\begin{tabular}{|c|c|c|}
\hline Source & Channel & \multicolumn{1}{|c|}{ Energy $(\mathrm{keV})$} \\
\hline${ }^{133} \mathrm{Ba} \varepsilon$ & 81.3652 & $53.1622 \pm 0.0006$ \\
${ }^{133} \mathrm{Ba} \varepsilon$ & 127.685 & $160.6120 \pm 0.0016$ \\
${ }^{133} \mathrm{Ba} \varepsilon$ & 154.316 & $223.2368 \pm 0.0013$ \\
${ }^{133} \mathrm{Ba} \varepsilon$ & 176.969 & $276.3989 \pm 0.0012$ \\
${ }^{133} \mathrm{Ba} \varepsilon$ & 188.223 & $302.8508 \pm 0.0005$ \\
${ }^{133} \mathrm{Ba} \varepsilon$ & 210.917 & $356.0129 \pm 0.0007$ \\
${ }^{133} \mathrm{Ba} \varepsilon$ & 222.775 & $383.8485 \pm 0.0012$ \\
${ }^{60} \mathrm{Co} \beta^{-}$ & 558.9 & $1173.228 \pm 0.003$ \\
${ }^{60} \mathrm{Co} \beta^{-}$ & 626.754 & $1332.492 \pm 0.004$ \\
${ }^{1} \mathrm{H}(n, \gamma)$ & 1006.12 & $2223.245 \pm 0.003$ \\
${ }^{208} \mathrm{~Pb}\left(n, n^{\prime} \gamma\right)$ & 1172.79 & $2614.511 \pm 0.010$ \\
${ }^{56} \mathrm{Fe}(n, \gamma)$ & 3310.73 & $7631.18 \pm 0.10$ \\
${ }^{56} \mathrm{Fe}(n, \gamma)$ & 3316.91 & $7645.58 \pm 0.10$ \\
\hline
\end{tabular}

Table 5.2: Energy calibration parameters, as per equation (4.1).

\begin{tabular}{|c|c|}
\hline Parameter & Value \\
\hline$a_{0}$ & $(-3.4 \pm 1.3) \times 10^{-7}$ \\
$a_{1}$ & $2.3481 \pm 0.0005$ \\
$a_{2}$ & $-138.97 \pm 0.19$ \\
\hline
\end{tabular}

\subsection{Rays and $\gamma$ Rays After Muon Capture on Uranium}

Table 5.3 contains a list of the identified peaks $[7,18,40]$. Figure 5.1 is a graphic of the full uranium spectrum. Identifications with just an isotope named are $\gamma$ rays from radioactive decay. Identifications prefaced with $\mu^{-}$are muonic $\mathrm{x}$ rays. Identifications of the form $(\mathrm{n}, \gamma)$ are $\gamma$ rays produced by a neutron interacting with the element's nucleus. Table 5.4 contains a list of the identified muonic $\mathrm{x}$ rays.

Some of the identifications in Table 5.3 are suspect. Some peaks, such as the $112.6 \mathrm{keV}{ }^{234} \mathrm{Th}$, typically indicate that other peaks should be seen $(63.29 \mathrm{keV}$ and $92.38 \mathrm{keV}$ in this case), which have not been detected in the spectrum. Similarly, the relative intensities of peaks should match up with the literature. To test for this, the 
observed relative intensities of the peaks are compared with the relative intensities of the literature values. For example, the $185.7 \mathrm{keV}, 258.6 \mathrm{keV}, 258.6 \mathrm{keV}, 743 \mathrm{keV}$, $766.6 \mathrm{keV}, 786.7 \mathrm{keV}, 882.2 \mathrm{keV}, 925.4 \mathrm{keV}, 946.1 \mathrm{keV}, 1061.7 \mathrm{keV}, 1127.7 \mathrm{keV}, 1393.3 \mathrm{keV}$, $1695.2 \mathrm{keV}, 2268 \mathrm{keV}$ and $2922.7 \mathrm{keV}$ all have intensities much higher than the literature values would otherwise suggest. Finally, there are some peaks that have been identified as single escape $(882.2 \mathrm{keV}, 1015.2 \mathrm{keV}$ and $1570.67 \mathrm{keV})$ that are suspect because, while pair-production is possible at these energies, the cross-section is quite low.

This problem with the relative intensities could be due to a partial correct identification combined with gamma rays from other fission products. Tables of gamma rays from fission products from other nuclear reactions on ${ }^{238} \mathrm{U}$ were consulted to identify some of these fission products, but none with the appropriate intensities were found $[41,42]$. Other references could be consulted in future work [43, 44, 45]. 


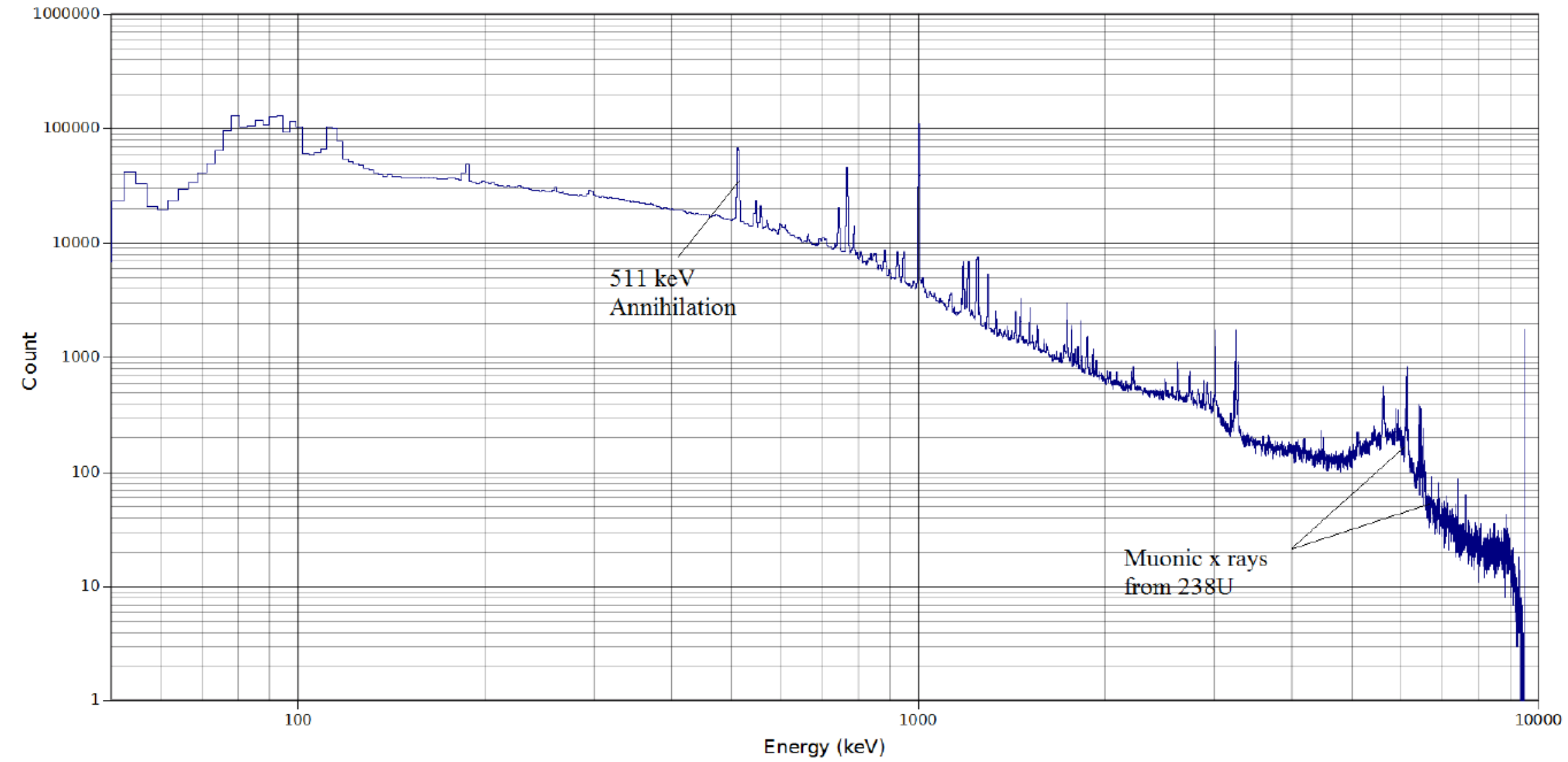

Figure 5.1: The full ${ }^{238} \mathrm{U}$ spectrum. Data below $50 \mathrm{keV}$ are not shown as they were not used anywhere in the spectrum analysis. 
Table 5.3: Peaks associated only with the active-beam run. All peaks in common with the background run have not been included in this table. The identifications marked with $\dagger$ are suspect as they have intensities much higher than would be expected. The identifications marked with $\ddagger$ are suspect as they have intensities much lower than the literature values would suggest. The single escape (SE) peaks marked with $*$ are suspect as the cross-section for pair production would be low in these energy ranges.

\begin{tabular}{|c|c|c|c|}
\hline Energy (keV) & Expected Energy (keV) & Identification & Number of Counts \\
\hline $112.6 \pm 0.2$ & 112.81 & ${ }^{234} \mathrm{Th} ?$ & $(1.340 \pm 0.011) \times 10^{5}$ \\
\hline $185.7 \pm 0.2$ & 186.15 & ${ }^{234} \mathrm{~Pa} \dagger$ & $(2.19 \pm 0.07) \times 10^{4}$ \\
\hline \multirow[t]{2}{*}{$258.6 \pm 0.2$} & 258.227 & ${ }^{234} \mathrm{~Pa} \dagger$ & $(5.4 \pm 0.6) \times 10^{3}$ \\
\hline & 258.86 & ${ }^{214} \mathrm{~Pb} \dagger$ & \\
\hline $295.5 \pm 0.3$ & \multirow[t]{2}{*}{295.228} & \multirow[t]{2}{*}{${ }^{214} \mathrm{~Pb} \ddagger$} & $(8.2 \pm 0.6) \times 10^{3}$ \\
\hline $369.8 \pm 0.6$ & & & $(1.1 \pm 0.5) \times 10^{3}$ \\
\hline $470.7 \pm 0.5$ & 469.77 & ${ }^{214} \mathrm{Bif}$ & $(1.3 \pm 0.4) \times 10^{3}$ \\
\hline $547.2 \pm 0.2$ & 548.67 & DE of $1570.67 *$ & $(6.27 \pm 0.13) \times 10^{4}$ \\
\hline $555.3 \pm 0.2$ & 554.75 & $\mu^{-238} \mathrm{U} 5 g \rightarrow 4 f$ & $(1.21 \pm 0.04) \times 10^{4}$ \\
\hline $569.8 \pm 0.3$ & 569.5 & ${ }^{234} \mathrm{~Pa}$ & $(4.7 \pm 0.5) \times 10^{3}$ \\
\hline $642.2 \pm 1.7$ & & & $(3 \pm 2) \times 10^{3}$ \\
\hline $743.0 \pm 0.3$ & 742.813 & ${ }^{234} \mathrm{~Pa} \dagger$ & $(1.93 \pm 0.04) \times 10^{4}$ \\
\hline $766.6 \pm 0.3$ & 766.42 & ${ }^{234} \mathrm{~Pa} \dagger$ & $(6.12 \pm 0.05) \times 10^{4}$ \\
\hline \multirow[t]{3}{*}{$786.7 \pm 0.3$} & 786.26 & ${ }^{234} \mathrm{~Pa} \dagger$ & $(1.0 \pm 0.4) \times 10^{4}$ \\
\hline & 785.96 & ${ }^{214} \mathrm{~Pb}$ & \\
\hline & 786.35 & ${ }^{214} \mathrm{Bi}$ & \\
\hline \multirow[t]{2}{*}{$806.8 \pm 0.3$} & 805.8 & ${ }^{234} \mathrm{~Pa}$ & $(2.5 \pm 0.3) \times 10^{3}$ \\
\hline & 805.74 & ${ }^{234 \mathrm{~m}} \mathrm{~Pa}$ & \\
\hline \multicolumn{4}{|c|}{ Continued on next page } \\
\hline
\end{tabular}


Table 5.3 - continued from previous page

\begin{tabular}{|c|c|c|c|}
\hline Energy $(\mathrm{keV})$ & Expected Energy (keV) & Identification & Number of Counts \\
\hline \multirow[t]{3}{*}{$882.2 \pm 0.3$} & 883.24 & ${ }^{234 \mathrm{~m}} \mathrm{~Pa} \dagger$ & $(7.6 \pm 0.4) \times 10^{3}$ \\
\hline & 881.7 & SE of $1392.7 *$ & \\
\hline & 881.5 & SE of $1392.5 *$ & \\
\hline \multirow{2}{*}{$925.4 \pm 0.3$} & 925.0 & ${ }^{234} \mathrm{~Pa}$ & $(8.3 \pm 0.4) \times 10^{3}$ \\
\hline & 926.61 & ${ }^{234 \mathrm{~m}} \mathrm{~Pa} \dagger$ & \\
\hline \multirow{2}{*}{$946.1 \pm 0.3$} & 945.90 & ${ }^{234 \mathrm{~m}} \mathrm{~Pa} \dagger$ & $(6.7 \pm 0.4) \times 10^{3}$ \\
\hline & 946.00 & ${ }^{234} \mathrm{~Pa}$ & \\
\hline $980.2 \pm 0.5$ & 980.3 & ${ }^{234} \mathrm{~Pa}$ & $(6 \pm 2) \times 10^{2}$ \\
\hline $1001.1 \pm 0.3$ & 1001.03 & ${ }^{234 \mathrm{~m}} \mathrm{~Pa}$ & $(1.662 \pm 0.007) \times 10^{5}$ \\
\hline $1015.2 \pm 0.4$ & 1016.27 & $\mathrm{SE}$ of ${ }^{234 \mathrm{~m}} \mathrm{~Pa} *$ & $(1.4 \pm 0.2) \times 10^{3}$ \\
\hline $1027.7 \pm 0.6$ & 1028.7 & ${ }^{234} \mathrm{~Pa}$ & $(6 \pm 3) \times 10^{2}$ \\
\hline $1061.7 \pm 0.7$ & 1061.86 & ${ }^{234 \mathrm{~m}} \mathrm{~Pa} \dagger$ & $(1.3 \pm 0.5) \times 10^{3}$ \\
\hline $1127.7 \pm 0.4$ & 1125.7 & ${ }^{234 \mathrm{~m}} \mathrm{~Pa} \dagger$ & $(3.0 \pm 0.4) \times 10^{3}$ \\
\hline $1141.1 \pm 0.4$ & & & $(4.8 \pm 1.5) \times 10^{2}$ \\
\hline $1180.7 \pm 0.3$ & 1181.07 & $\mu^{-238} \mathrm{U} 4 f \rightarrow 3 d$ & $(1.06 \pm 0.04) \times 10^{4}$ \\
\hline $1220.5 \pm 0.4$ & 1220.37 & ${ }^{234 \mathrm{~m}} \mathrm{~Pa}$ & $(5 \pm 6) \times 10^{2}$ \\
\hline $1244.1 \pm 0.3$ & 1243.94 & $\mu^{-238} \mathrm{U} 4 f \rightarrow 3 d$ & $(1.08 \pm 0.03) \times 10^{4}$ \\
\hline $1257.6 \pm 0.6$ & & & $(7 \pm 3) \times 10^{2}$ \\
\hline $1353.0 \pm 0.8$ & 1352.9 & ${ }^{234} \mathrm{~Pa}$ & $(2.5 \pm 1.0) \times 10^{2}$ \\
\hline \multirow[t]{3}{*}{$1393.3 \pm 0.5$} & 1392.7 & ${ }^{234 \mathrm{~m}} \mathrm{~Pa}$ & $(9.1 \pm 1.6) \times 10^{2}$ \\
\hline & 1392.5 & ${ }^{214} \mathrm{Bi} \dagger$ & \\
\hline & 1393.9 & ${ }^{234} \mathrm{~Pa}$ & \\
\hline $1434.0 \pm 0.4$ & 1434.14 & ${ }^{234 \mathrm{~m}} \mathrm{~Pa}$ & $(2.12 \pm 0.19) \times 10^{3}$ \\
\hline $1527.5 \pm 0.5$ & 1527.27 & ${ }^{234 \mathrm{~m}} \mathrm{~Pa}$ & $(4.5 \pm 1.5) \times 10^{2}$ \\
\hline
\end{tabular}


Table 5.3 - continued from previous page

\begin{tabular}{|c|c|c|c|}
\hline Energy (keV) & Expected Energy (keV) & Identification & Number of Counts \\
\hline $1553.7 \pm 0.4$ & 1553.74 & ${ }^{234 \mathrm{~m}} \mathrm{~Pa}$ & $(1.1 \pm 0.3) \times 10^{3}$ \\
\hline $1568.9 \pm 0.9$ & 1570.67 & ${ }^{234 \mathrm{~m}} \mathrm{~Pa}$ & $(1.5 \pm 0.9) \times 10^{2}$ \\
\hline $1592.8 \pm 0.6$ & 1593.88 & ${ }^{234 \mathrm{~m}} \mathrm{~Pa}$ & $(6.8 \pm 1.6) \times 10^{2}$ \\
\hline $1609.5 \pm 0.6$ & & & $(3.4 \pm 1.3) \times 10^{2}$ \\
\hline $1695.2 \pm 0.8$ & 1694.1 & ${ }^{234 \mathrm{~m}} \mathrm{~Pa} \dagger$ & $(6.4 \pm 1.5) \times 10^{2}$ \\
\hline $1720.0 \pm 0.7$ & & & $(1.0 \pm 0.2) \times 10^{3}$ \\
\hline $1737.5 \pm 0.4$ & 1737.75 & ${ }^{234 \mathrm{~m}} \mathrm{~Pa}$ & $(3.28 \pm 0.15) \times 10^{3}$ \\
\hline $1790.8 \pm 0.5$ & & & $(6 \pm 2) \times 10^{2}$ \\
\hline $1867.0 \pm 0.5$ & 1867.68 & ${ }^{234 \mathrm{~m}} \mathrm{~Pa}$ & $(8.7 \pm 1.0) \times 10^{2}$ \\
\hline $1874.8 \pm 0.7$ & 1874.88 & ${ }^{234 \mathrm{~m}} \mathrm{~Pa}$ & $(1.70 \pm 0.43) \times 10^{3}$ \\
\hline $1893.6 \pm 0.7$ & 1893.51 & ${ }^{234 \mathrm{~m}} \mathrm{~Pa}$ & $(4.9 \pm 1.3) \times 10^{2}$ \\
\hline $1911.0 \pm 0.5$ & 1911.19 & ${ }^{234 \mathrm{~m}} \mathrm{~Pa}$ & $(9.8 \pm 1.2) \times 10^{2}$ \\
\hline \multirow[t]{2}{*}{$1936.7 \pm 0.5$} & 1937.04 & ${ }^{234 \mathrm{~m}} \mathrm{~Pa}$ & $(4 \pm 2) \times 10^{2}$ \\
\hline & 1935.62 & ${ }^{214} \mathrm{Bi}$ & \\
\hline $2015.3 \pm 0.7$ & & & $(2.4 \pm 0.7) \times 10^{2}$ \\
\hline $2039.6 \pm 0.7$ & & & $(2.3 \pm 1.4) \times 10^{2}$ \\
\hline $2088.6 \pm 0.8$ & 2085.19 & ${ }^{214} \mathrm{Bi}$ & $(4.6 \pm 1.3) \times 10^{2}$ \\
\hline $2144.5 \pm 1.5$ & & & $(2.0 \pm 1.3) \times 10^{2}$ \\
\hline $2159.6 \pm 1.0$ & & & $(1.2 \pm 1.9) \times 10^{2}$ \\
\hline $2268 \pm 8$ & 2270.9 & ${ }^{214} \mathrm{Bi} \dagger$ & $(2 \pm 6) \times 10^{2}$ \\
\hline $2443.9 \pm 1.8$ & & & $(1.5 \pm 0.9) \times 10^{2}$ \\
\hline $2501.8 \pm 1.3$ & & $\mathrm{SE}$ of 3013.2 & $(7 \pm 3) \times 10^{2}$ \\
\hline $2519.8 \pm 1.2$ & & & $(9 \pm 6) \times 10^{1}$ \\
\hline $2564.5 \pm 0.9$ & & & $(1.4 \pm 0.6) \times 10^{2}$ \\
\hline & & & ntinued on next page \\
\hline
\end{tabular}


Table 5.3 - continued from previous page

\begin{tabular}{|c|c|c|c|}
\hline Energy $(\mathrm{keV})$ & Expected Energy (keV) & Identification & Number of Counts \\
\hline $2738.0 \pm 0.7$ & 2732 & $\begin{array}{l}\mu^{-98} \text { Mo candidate } \\
\left(2 p_{3 / 2} \rightarrow 1 s_{1 / 2}\right)\end{array}$ & $(1.05 \pm 0.15) \times 10^{3}$ \\
\hline $2883.1 \pm 0.8$ & & & $(8.8 \pm 1.2) \times 10^{2}$ \\
\hline $2922.7 \pm 0.9$ & 2921.98 & ${ }^{214} \mathrm{Bi} \dagger$ & $(1.18 \pm 0.15) \times 10^{3}$ \\
\hline $2956.9 \pm 1.2$ & & & $(6.0 \pm 1.8) \times 10^{2}$ \\
\hline $3013.2 \pm 0.7$ & 3013.02 & $\mu^{-238} \mathrm{U} 3 d \rightarrow 2 p$ & $(4.19 \pm 0.15) \times 10^{3}$ \\
\hline $3046.0 \pm 1.3$ & & & $(1.1 \pm 0.7) \times 10^{2}$ \\
\hline $3121.6 \pm 1.9$ & & & $(8 \pm 7) \times 10^{1}$ \\
\hline $3192.1 \pm 0.9$ & & & $(3.0 \pm 0.7) \times 10^{2}$ \\
\hline $3222.4 \pm 0.8$ & & & $(8.4 \pm 1.1) \times 10^{2}$ \\
\hline $3248.9 \pm 0.8$ & & & $(4.91 \pm 0.15) \times 10^{3}$ \\
\hline $3277.6 \pm 0.8$ & 3277.44 & $\mu^{-238} \mathrm{U} 3 d \rightarrow 2 p$ & $(3.5 \pm 0.2) \times 10^{3}$ \\
\hline $3900.2 \pm 1.7$ & 3899.1 & $\mu^{-133} \mathrm{Cs}$ candidate & $(5 \pm 5) \times 10^{1}$ \\
\hline & 3902.18 & $\left(2 p_{3 / 2} \rightarrow 1 s_{1 / 2}\right)$ & \\
\hline $3971.9 \pm 1.7$ & & & $(6 \pm 5) \times 10^{1}$ \\
\hline $4194.7 \pm 1.2$ & & & $(1.3 \pm 0.5) \times 10^{2}$ \\
\hline $4466.1 \pm 1.2$ & & & $(2.5 \pm 0.7) \times 10^{2}$ \\
\hline $5116 \pm 3$ & & SE of 5606.9 & $(2 \pm 1) \times 10^{2}$ \\
\hline $5606.9 \pm 1.5$ & & & $(8.8 \pm 1.5) \times 10^{2}$ \\
\hline $5625.9 \pm 1.5$ & & & $(6.3 \pm 1.0) \times 10^{2}$ \\
\hline $5862 \pm 2$ & & & $(1.0 \pm 0.7) \times 10^{2}$ \\
\hline $5894.2 \pm 1.7$ & & & $(6.8 \pm 1.4) \times 10^{2}$ \\
\hline $5918.2 \pm 1.9$ & & & $(9 \pm 7) \times 10^{1}$ \\
\hline $5939.8 \pm 1.6$ & 5943.14 & SE of 6454.14 & $(5.9 \pm 1.0) \times 10^{2}$ \\
\hline
\end{tabular}


Table 5.3 - continued from previous page

\begin{tabular}{|c|c|c|c|}
\hline Energy (keV) & Expected Energy (keV) & Identification & Number of Counts \\
\hline $5966.6 \pm 1.7$ & 5969.93 & SE of 6480.93 & $(1.0 \pm 0.6) \times 10^{2}$ \\
\hline $6005 \pm 2$ & 6007.36 & SE of 6518.36 & $(2.5 \pm 1.0) \times 10^{2}$ \\
\hline $6048 \pm 3$ & 6047.36 & $\mu^{-238} \mathrm{U} 2 p \rightarrow 1 s$ & $(3 \pm 7) \times 10^{1}$ \\
\hline $6093.6 \pm 1.6$ & 6096.58 & $\mu^{-238} \mathrm{U} 2 p \rightarrow 1 s$ & $(5.0 \pm 0.9) \times 10^{2}$ \\
\hline $6119.0 \pm 1.6$ & 6122.1 & $\mu^{-238} \mathrm{U} 2 p \rightarrow 1 s$ & $(1.29 \pm 0.10) \times 10^{3}$ \\
\hline $6138.7 \pm 1.6$ & & & $(2.49 \pm 0.18) \times 10^{3}$ \\
\hline $6163.8 \pm 1.7$ & 6167.41 & $\mu^{-238} \mathrm{U} 2 p \rightarrow 1 s$ & $(2.5 \pm 0.6) \times 10^{2}$ \\
\hline $6377 \pm 2$ & 6379.26 & $\mu^{-238} \mathrm{U} 2 p \rightarrow 1 s$ & $(1.2 \pm 0.5) \times 10^{2}$ \\
\hline $6406.3 \pm 1.7$ & 6409.44 & $\mu^{-238} \mathrm{U} 2 p \rightarrow 1 s$ & $(9.2 \pm 0.9) \times 10^{2}$ \\
\hline $6451.2 \pm 1.7$ & 6454.14 & $\mu^{-238} \mathrm{U} 2 p \rightarrow 1 s$ & $(1.03 \pm 0.08) \times 10^{3}$ \\
\hline $6478.8 \pm 1.8$ & 6480.93 & $\mu^{-238} \mathrm{U} 2 p \rightarrow 1 s$ & $(2.9 \pm 0.5) \times 10^{2}$ \\
\hline $6515.7 \pm 1.8$ & 6518.36 & $\mu^{-238} \mathrm{U} 2 p \rightarrow 1 s$ & $(4.1 \pm 0.7) \times 10^{2}$ \\
\hline $6560 \pm 2$ & 6563.7 & $\mu^{-238} \mathrm{U} 2 p \rightarrow 1 s$ & $(3.6 \pm 1.3) \times 10^{2}$ \\
\hline $6903 \pm 2$ & & SE of 7416 & $(1.1 \pm 0.4) \times 10^{2}$ \\
\hline $7416 \pm 2$ & & & $(1.5 \pm 0.3) \times 10^{2}$ \\
\hline $7630 \pm 2$ & 7631.18 & ${ }^{56} \mathrm{Fe}(n, \gamma)$ & $(7 \pm 2) \times 10^{1}$ \\
\hline $7646 \pm 3$ & 7645.58 & ${ }^{56} \mathrm{Fe}(n, \gamma)$ & $(5 \pm 4) \times 10^{1}$ \\
\hline $9291 \pm 3$ & & & $(5 \pm 2) \times 10^{1}$ \\
\hline
\end{tabular}

\subsubsection{Muonic X Rays from Fission Products}

A list of the muonic $\mathrm{x}$ rays produced by fission products can be found in Table 5.5. This includes the upper limits on the identification of muonic $\mathrm{x}$ rays. In most cases, the upper limit is zero, as there was no peak distinguishable from the background. The two candidate events with a positive area are shown in Figures 5.2 and 5.3. The 
Table 5.4: $\mu^{-} \mathrm{X}$ rays identified from uranium.

\begin{tabular}{|c|c|c|c|}
\hline Energy (keV) & Expected Energy (keV) & Identification & Number of counts \\
\hline $555.3 \pm 0.2$ & 554.75 & $\mu^{-238} \mathrm{U} 5 g \rightarrow 4 f$ & $(1.21 \pm 0.04) \times 10^{4}$ \\
$1180.7 \pm 0.3$ & 1181.07 & $\mu^{-238} \mathrm{U} 4 f \rightarrow 3 d$ & $(1.06 \pm 0.04) \times 10^{4}$ \\
$1244.1 \pm 0.3$ & 1243.94 & $\mu^{-238} \mathrm{U} 4 f \rightarrow 3 d$ & $(1.08 \pm 0.03) \times 10^{4}$ \\
$3013.2 \pm 0.7$ & 3013.02 & $\mu^{-238} \mathrm{U} 3 d \rightarrow 2 p$ & $(4.19 \pm 0.15) \times 10^{3}$ \\
$3277.6 \pm 0.8$ & 3277.44 & $\mu^{-238} \mathrm{U} 3 d \rightarrow 2 p$ & $(3.5 \pm 0.2) \times 10^{3}$ \\
$6048 \pm 3$ & 6047.36 & $\mu^{-238} \mathrm{U} 2 p \rightarrow 1 s$ & $(3 \pm 7) \times 10^{1}$ \\
$6093.6 \pm 1.6$ & 6096.58 & $\mu^{-238} \mathrm{U} 2 p \rightarrow 1 s$ & $(5.0 \pm 0.9) \times 10^{2}$ \\
$6119.0 \pm 1.6$ & 6122.1 & $\mu^{-238} \mathrm{U} 2 p \rightarrow 1 s$ & $(1.29 \pm 0.10) \times 10^{3}$ \\
$6163.8 \pm 1.7$ & 6167.41 & $\mu^{-238} \mathrm{U} 2 p \rightarrow 1 s$ & $(2.5 \pm 0.6) \times 10^{2}$ \\
$6377 \pm 2$ & 6379.26 & $\mu^{-238} \mathrm{U} 2 p \rightarrow 1 s$ & $(1.2 \pm 0.5) \times 10^{2}$ \\
$6406.3 \pm 1.7$ & 6409.44 & $\mu^{-238} \mathrm{U} 2 p \rightarrow 1 s$ & $(9.2 \pm 0.9) \times 10^{2}$ \\
$6451.2 \pm 1.7$ & 6454.14 & $\mu^{-238} \mathrm{U} 2 p \rightarrow 1 s$ & $(1.03 \pm 0.08) \times 10^{3}$ \\
$6478.8 \pm 1.8$ & 6480.93 & $\mu^{-238} \mathrm{U} 2 p \rightarrow 1 s$ & $(2.9 \pm 0.5) \times 10^{2}$ \\
$6515.7 \pm 1.8$ & 6518.36 & $\mu^{-238} \mathrm{U} 2 p \rightarrow 1 s$ & $(4.1 \pm 0.7) \times 10^{2}$ \\
$6560 \pm 2$ & 6563.7 & $\mu^{-238} \mathrm{U} 2 p \rightarrow 1 s$ & $(3.6 \pm 1.3) \times 10^{2}$ \\
\hline
\end{tabular}

second candidate does not have a statistically significant peak, but is included for analysis.

Table 5.5: Candidate muonic x rays identified from uranium fission products. Upper limits are given for x rays where no clear peak was identifed. All x ray candidates are $2 p_{3 / 2} \rightarrow 1 s_{1 / 2}$ transitions.

\begin{tabular}{|c|c|c|c|}
\hline Energy (keV) & Expected Energy (keV) & Identification & $\begin{array}{c}\text { Number of Counts } \\
\text { or Upper Limit }\end{array}$ \\
\hline $2738.0 \pm 0.7$ & 2732.00 & $\mu^{-98} \mathrm{Mo}$ X ray & $(1.10 \pm 0.15) \times 10^{3}$ \\
& 2982.00 & $\mu^{-103} \mathrm{Rh} \mathrm{X}$ ray & $<200$ \\
& 3077.00 & $\mu^{- \text {nat } \mathrm{Pd} \text { X ray }}$ & $<170$ \\
$3900.2 \pm 1.7$ & 3723.00 & $\mu^{-127} \mathrm{I}$ ray & $<128$ \\
& 3899.1 & $\mu^{-133} \mathrm{Cs} \mathrm{X}$ ray & $50 \pm 50$ \\
& 3902.18 & & \\
& 4335.00 & $\mu^{- \text {nat } \mathrm{Nd} \text { X ray }}$ & $<112$ \\
\hline
\end{tabular}

\section{$5.3 \quad$ Acceptances}

The acceptance is needed to properly calculate the yield of the fission product candidate $\mathrm{x}$ rays, and to obtain an estimate on the number of counts that should be in 


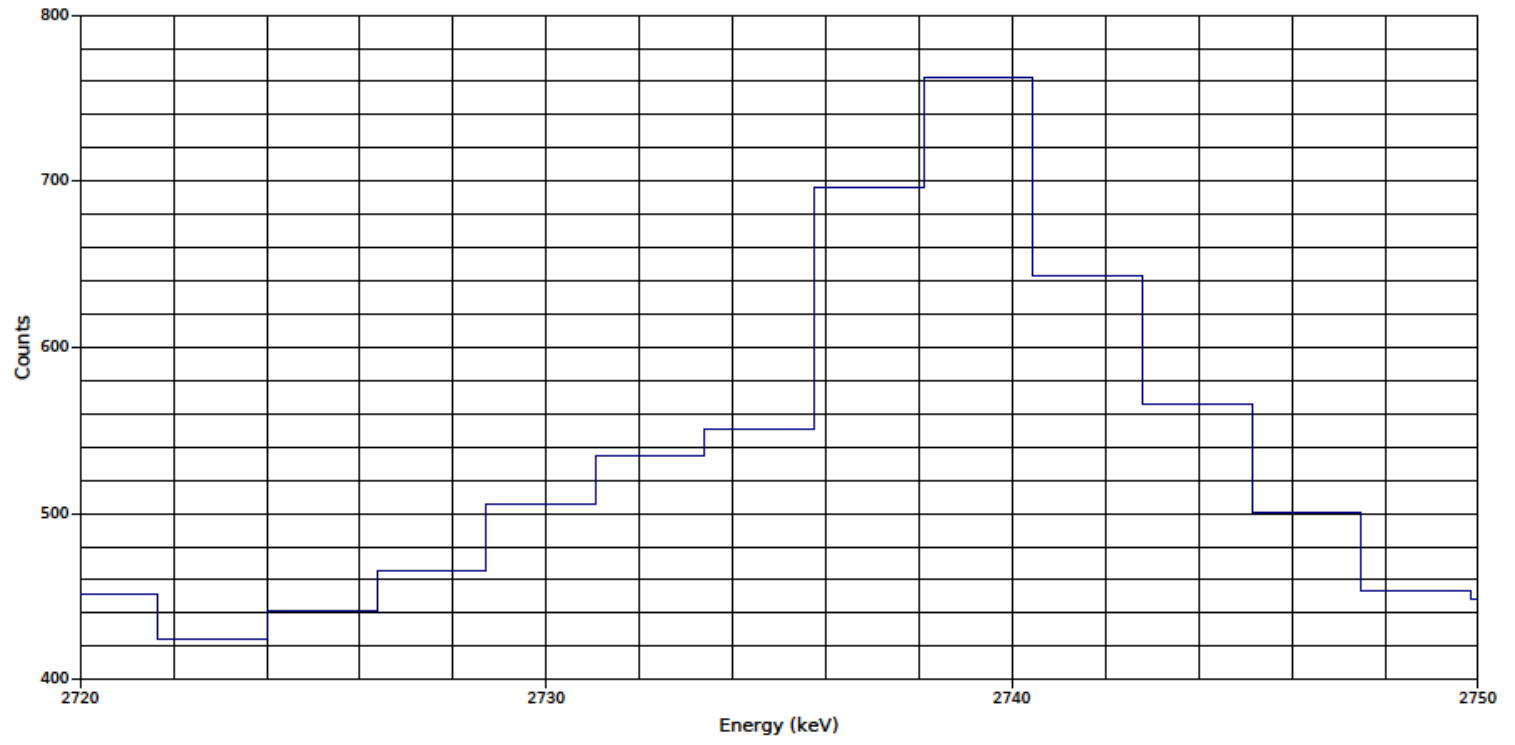

Figure 5.2: The candidate peak for the $\mu^{-98}$ Mo $\mathrm{x}$ ray.

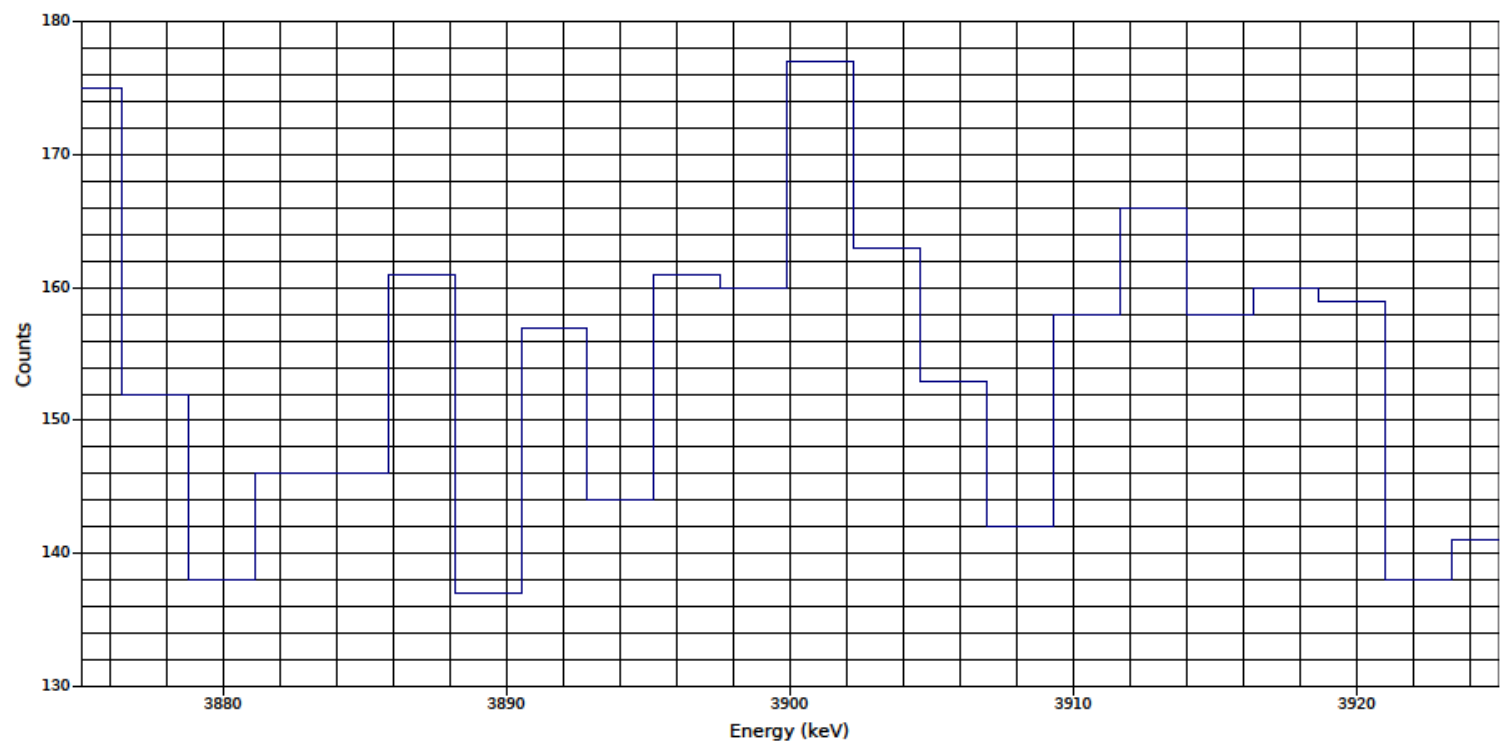

Figure 5.3: The candidate for the $\mu^{-137} \mathrm{Cs} \mathrm{x}$ ray. The "peak" is not statistically significant. 
these peaks. As such, verifying that the acceptances have been properly calculated is of value, to ensure that further calculations are valid.

The results of the Monte Carlo acceptance simulation are shown graphically in Figure 5.4. Table 3.3 contains the experimental acceptance calculated previously by Olsthorn [4]. In Table 5.6, we can see that the percent difference between the simulation and the experiment is at most $14 \%$. The agreement factor is defined in equation (5.1) and the percent difference is defined in equation (5.2), where $s$ and $e$ refer to the simulation and the experiment respectively. The agreement factor is the number of sigma away from zero that the difference between the two values is. The fit used is an empirical equation - a third-order polynomial of the form seen in Equation (5.3).

$$
\begin{gathered}
\text { Agreement }=\frac{\xi_{e}-\xi_{s}}{\sqrt{\sigma_{e}^{2}+\sigma_{s}^{2}}} \\
\text { \%diff }=\frac{\xi_{e}-\xi_{s}}{\xi_{e}} \cdot 100 \% \\
\ln (\xi)=\sum_{i=0}^{3} \ln (E)^{i}
\end{gathered}
$$

The logbook suggests that a scintillator was present, and the systematically higher simulated acceptances suggest this as well. However, the thickness of this scintillator was not known. Thus, three additional simulations were performed, for three possible thicknesses of the scintillator: $0.15875 \mathrm{~cm}, 0.3175 \mathrm{~cm}$ and $0.5 \mathrm{~cm}$. The results of these additional simulations can be seen in Tables 5.7, 5.8, and 5.9.

\subsection{Dose from Muons}

Using a momentum of $80 \mathrm{MeV} / \mathrm{c}$ (the momentum used in the experiment), the maximum kinetic energy of a muon is $26.6 \mathrm{MeV}$. The data from Table 5.10 were obtained 
Table 5.6: Simulation acceptance and comparison with experimental acceptance. No electron veto scintillator was present.

\begin{tabular}{|l|l|l|l|l|}
\hline $\begin{array}{l}\text { Energy } \\
(\mathrm{keV})\end{array}$ & Source & $\begin{array}{l}\text { Simulation } \\
\text { Acceptance }\end{array}$ & $\begin{array}{l}\text { Percent } \\
\text { Difference }\end{array}$ & $\begin{array}{l}\text { Agreement } \\
\text { Factor }\end{array}$ \\
\hline 121.7817 & ${ }^{152} \mathrm{Eu}$ & $(3.362 \pm 0.004) \times 10^{-3}$ & $13.57 \%$ & 14.19 \\
244.6875 & ${ }^{152} \mathrm{Eu}$ & $(2.780 \pm 0.004) \times 10^{-3}$ & $-1.41 \%$ & -1.42 \\
276.3997 & ${ }^{133} \mathrm{Ba}$ & $(2.641 \pm 0.004) \times 10^{-3}$ & $-7.26 \%$ & -5.56 \\
302.851 & ${ }^{133} \mathrm{Ba}$ & $(2.530 \pm 0.003) \times 10^{-3}$ & $-7.36 \%$ & -8.98 \\
344.2785 & ${ }^{152} \mathrm{Eu}$ & $(2.389 \pm 0.003) \times 10^{-3}$ & $-1.49 \%$ & -2.27 \\
356.0134 & ${ }^{133} \mathrm{Ba}$ & $(2.340 \pm 0.003) \times 10^{-3}$ & $-6.19 \%$ & -14.93 \\
383.848 & ${ }^{133} \mathrm{Ba}$ & $(2.266 \pm 0.003) \times 10^{-3}$ & $-7.31 \%$ & -8.44 \\
411.1163 & ${ }^{152} \mathrm{Eu}$ & $(2.196 \pm 0.003) \times 10^{-3}$ & $-6.48 \%$ & -3.03 \\
443.965 & ${ }^{152} \mathrm{Eu}$ & $(2.116 \pm 0.003) \times 10^{-3}$ & $-2.13 \%$ & -1.25 \\
778.904 & ${ }^{152} \mathrm{Eu}$ & $(1.623 \pm 0.003) \times 10^{-3}$ & $-2.55 \%$ & -2.64 \\
867.373 & ${ }^{152} \mathrm{Eu}$ & $(1.539 \pm 0.003) \times 10^{-3}$ & $-4.37 \%$ & -3.04 \\
964.079 & ${ }^{152} \mathrm{Eu}$ & $(1.472 \pm 0.003) \times 10^{-3}$ & $-2.95 \%$ & -3.71 \\
1112.069 & ${ }^{152} \mathrm{Eu}$ & $(1.368 \pm 0.003) \times 10^{-3}$ & $-0.65 \%$ & -0.14 \\
1173.228 & ${ }^{60} \mathrm{Co}$ & $(1.368 \pm 0.003) \times 10^{-3}$ & $0.16 \%$ & 0.15 \\
1212.948 & ${ }^{152} \mathrm{Eu}$ & $(1.313 \pm 0.003) \times 10^{-3}$ & $-1.54 \%$ & -0.55 \\
1274.537 & ${ }^{22} \mathrm{Na}$ & $(1.281 \pm 0.003) \times 10^{-3}$ & $-5.5 \%$ & -6.45 \\
1299.142 & $152 \mathrm{Eu}$ & $(1.266 \pm 0.003) \times 10^{-3}$ & $-12.12 \%$ & -4.7 \\
1332.492 & ${ }^{60} \mathrm{Co}$ & $(1.252 \pm 0.003) \times 10^{-3}$ & $-0.66 \%$ & -0.62 \\
1408.006 & ${ }^{152} \mathrm{Eu}$ & $(1.215 \pm 0.003) \times 10^{-3}$ & $-2.48 \%$ & -3.24 \\
2341.2 & $\mu^{-} \mathrm{Au}$ & $(8.54 \pm 0.02) \times 10^{-4}$ & $-13.83 \%$ & -0.8 \\
2477.8 & $\mu^{-} \mathrm{Au}$ & $(8.24 \pm 0.02) \times 10^{-4}$ & $-12.84 \%$ & -0.62 \\
5591 & $\mu^{-} \mathrm{Au}$ & $(4.27 \pm 0.02) \times 10^{-4}$ & $-0.25 \%$ & -0.01 \\
5763.1 & $\mu^{-} \mathrm{Au}$ & $(4.16 \pm 0.02) \times 10^{-4}$ & $3.33 \%$ & 0.1 \\
\hline
\end{tabular}

from the simulation described in section 4.5. As can be seen in Table 5.10, the muons will stop within the phantom (i.e. all energy deposited) unless the muon has an energy in excess of several hundred MeV. Table 5.11 contains the dose equivalent deposited by a single muon into a phantom of a human of mass $70 \mathrm{~kg}$ and is obtained from applying equation 4.7 to the data in Table 5.10. Finally, Table 5.12 contains the number of muons required to hit to obtain a dose limit of $1 \mathrm{mSv}$ and is obtained by applying equation (4.8) to the data in Table 5.11. 
Table 5.7: Simulation acceptance and comparison with experimental acceptance. An electron veto scintillator of depth $0.15875 \mathrm{~cm}$ was present in the model.

\begin{tabular}{|l|l|l|l|l|}
\hline $\begin{array}{l}\text { Energy } \\
(\mathrm{keV})\end{array}$ & Source & $\begin{array}{l}\text { Simulation } \\
\text { Acceptance }\end{array}$ & $\begin{array}{l}\text { Percent } \\
\text { Difference }\end{array}$ & $\begin{array}{l}\text { Agreement } \\
\text { Factor }\end{array}$ \\
\hline 121.7817 & ${ }^{152} \mathrm{Eu}$ & $(3.282 \pm 0.004) \times 10^{-3}$ & $15.62 \%$ & 16.34 \\
244.6875 & ${ }^{152} \mathrm{Eu}$ & $(2.730 \pm 0.004) \times 10^{-3}$ & $0.42 \%$ & 0.42 \\
276.3997 & ${ }^{133} \mathrm{Ba}$ & $(2.597 \pm 0.003) \times 10^{-3}$ & $-5.47 \%$ & -4.19 \\
302.851 & ${ }^{133} \mathrm{Ba}$ & $(2.497 \pm 0.003) \times 10^{-3}$ & $-5.98 \%$ & -7.3 \\
344.2785 & ${ }^{152} \mathrm{Eu}$ & $(2.346 \pm 0.003) \times 10^{-3}$ & $0.34 \%$ & 0.51 \\
356.0134 & ${ }^{133} \mathrm{Ba}$ & $(2.312 \pm 0.003) \times 10^{-3}$ & $-4.66 \%$ & -11.26 \\
383.848 & ${ }^{133} \mathrm{Ba}$ & $(2.235 \pm 0.003) \times 10^{-3}$ & $-5.83 \%$ & -6.73 \\
411.1163 & ${ }^{152} \mathrm{Eu}$ & $(2.159 \pm 0.003) \times 10^{-3}$ & $-4.69 \%$ & -2.19 \\
443.965 & ${ }^{152} \mathrm{Eu}$ & $(2.082 \pm 0.003) \times 10^{-3}$ & $-0.5 \%$ & -0.29 \\
778.904 & ${ }^{152} \mathrm{Eu}$ & $(1.602 \pm 0.003) \times 10^{-3}$ & $-1.21 \%$ & -1.26 \\
867.373 & ${ }^{152} \mathrm{Eu}$ & $(1.530 \pm 0.003) \times 10^{-3}$ & $-3.76 \%$ & -2.62 \\
964.079 & ${ }^{152} \mathrm{Eu}$ & $(1.452 \pm 0.003) \times 10^{-3}$ & $-1.51 \%$ & -1.9 \\
1112.069 & ${ }^{152} \mathrm{Eu}$ & $(1.356 \pm 0.003) \times 10^{-3}$ & $0.24 \%$ & 0.05 \\
1173.228 & ${ }^{60} \mathrm{Co}$ & $(1.354 \pm 0.003) \times 10^{-3}$ & $1.14 \%$ & 1.1 \\
1212.948 & ${ }^{152} \mathrm{Eu}$ & $(1.301 \pm 0.003) \times 10^{-3}$ & $-0.65 \%$ & -0.23 \\
1274.537 & ${ }^{22} \mathrm{Na}$ & $(1.261 \pm 0.003) \times 10^{-3}$ & $-3.87 \%$ & -4.54 \\
1299.142 & ${ }^{152} \mathrm{Eu}$ & $(1.260 \pm 0.003) \times 10^{-3}$ & $-11.57 \%$ & -4.49 \\
1332.492 & ${ }^{60} \mathrm{Co}$ & $(1.240 \pm 0.003) \times 10^{-3}$ & $0.32 \%$ & 0.3 \\
1408.006 & ${ }^{152} \mathrm{Eu}$ & $(1.205 \pm 0.003) \times 10^{-3}$ & $-1.64 \%$ & -2.14 \\
2341.2 & $\mu^{-} \mathrm{Au}$ & $(8.53 \pm 0.02) \times 10^{-4}$ & $-13.8 \%$ & -0.8 \\
2477.8 & $\mu^{-} \mathrm{Au}$ & $(8.21 \pm 0.02) \times 10^{-4}$ & $-12.52 \%$ & -0.61 \\
5591 & $\mu^{-} \mathrm{Au}$ & $(4.249 \pm 0.016) \times 10^{-4}$ & $0.25 \%$ & 0.01 \\
5763.1 & $\mu^{-} \mathrm{Au}$ & $(4.129 \pm 0.016) \times 10^{-4}$ & $3.98 \%$ & 0.11 \\
\hline
\end{tabular}


Table 5.8: Simulation acceptance and comparison with experimental acceptance. An electron veto scintillator of depth $0.3175 \mathrm{~cm}$ was present in the model.

\begin{tabular}{|l|l|l|l|l|}
\hline $\begin{array}{l}\text { Energy } \\
(\mathrm{keV})\end{array}$ & Source & $\begin{array}{l}\text { Simulation } \\
\text { Acceptance }\end{array}$ & $\begin{array}{l}\text { Percent } \\
\text { Difference }\end{array}$ & $\begin{array}{l}\text { Agreement } \\
\text { Factor }\end{array}$ \\
\hline 121.7817 & ${ }^{152} \mathrm{Eu}$ & $(3.206 \pm 0.004) \times 10^{-3}$ & $17.6 \%$ & 18.41 \\
244.6875 & ${ }^{152} \mathrm{Eu}$ & $(2.730 \pm 0.004) \times 10^{-3}$ & $0.42 \%$ & 0.42 \\
276.3997 & ${ }^{133} \mathrm{Ba}$ & $(2.544 \pm 0.003) \times 10^{-3}$ & $-3.33 \%$ & -2.55 \\
302.851 & ${ }^{133} \mathrm{Ba}$ & $(2.452 \pm 0.003) \times 10^{-3}$ & $-4.08 \%$ & -4.98 \\
344.2785 & ${ }^{152} \mathrm{Eu}$ & $(2.304 \pm 0.003) \times 10^{-3}$ & $2.11 \%$ & 3.23 \\
356.0134 & ${ }^{133} \mathrm{Ba}$ & $(2.270 \pm 0.003) \times 10^{-3}$ & $-2.77 \%$ & -6.69 \\
383.848 & ${ }^{133} \mathrm{Ba}$ & $(2.188 \pm 0.003) \times 10^{-3}$ & $-3.62 \%$ & -4.17 \\
411.1163 & ${ }^{152} \mathrm{Eu}$ & $(2.129 \pm 0.003) \times 10^{-3}$ & $-3.25 \%$ & -1.52 \\
443.965 & ${ }^{152} \mathrm{Eu}$ & $(2.052 \pm 0.003) \times 10^{-3}$ & $0.96 \%$ & 0.57 \\
778.904 & ${ }^{152} \mathrm{Eu}$ & $(1.582 \pm 0.003) \times 10^{-3}$ & $0.08 \%$ & 0.08 \\
867.373 & ${ }^{152} \mathrm{Eu}$ & $(1.510 \pm 0.003) \times 10^{-3}$ & $-2.4 \%$ & -1.67 \\
964.079 & ${ }^{152} \mathrm{Eu}$ & $(1.436 \pm 0.003) \times 10^{-3}$ & $-0.42 \%$ & -0.53 \\
1112.069 & ${ }^{152} \mathrm{Eu}$ & $(1.347 \pm 0.003) \times 10^{-3}$ & $0.86 \%$ & 0.19 \\
1173.228 & ${ }^{60} \mathrm{Co}$ & $(1.340 \pm 0.003) \times 10^{-3}$ & $2.16 \%$ & 2.07 \\
1212.948 & ${ }^{152} \mathrm{Eu}$ & $(1.288 \pm 0.003) \times 10^{-3}$ & $0.42 \%$ & 0.15 \\
1274.537 & ${ }^{22} \mathrm{Na}$ & $(1.261 \pm 0.003) \times 10^{-3}$ & $-3.87 \%$ & -4.54 \\
1299.142 & ${ }^{152} \mathrm{Eu}$ & $(1.244 \pm 0.003) \times 10^{-3}$ & $-10.22 \%$ & -3.96 \\
1332.492 & ${ }^{60} \mathrm{Co}$ & $(1.226 \pm 0.003) \times 10^{-3}$ & $1.45 \%$ & 1.36 \\
1408.006 & ${ }^{152} \mathrm{Eu}$ & $(1.194 \pm 0.003) \times 10^{-3}$ & $-0.68 \%$ & -0.89 \\
2341.2 & $\mu^{-} \mathrm{Au}$ & $(8.47 \pm 0.02) \times 10^{-4}$ & $-12.92 \%$ & -0.75 \\
2477.8 & $\mu^{-} \mathrm{Au}$ & $(8.13 \pm 0.02) \times 10^{-4}$ & $-11.31 \%$ & -0.55 \\
5591 & $\mu^{-} \mathrm{Au}$ & $(4.226 \pm 0.016) \times 10^{-4}$ & $0.8 \%$ & 0.04 \\
5763.1 & $\mu^{-} \mathrm{Au}$ & $(4.102 \pm 0.016) \times 10^{-4}$ & $4.6 \%$ & 0.13 \\
\hline
\end{tabular}


Table 5.9: Simulation acceptance and comparison with experimental acceptance. An electron veto scintillator of depth $0.5 \mathrm{~cm}$ was present in the model.

\begin{tabular}{|l|l|l|l|l|}
\hline $\begin{array}{l}\text { Energy } \\
(\mathrm{keV})\end{array}$ & Source & $\begin{array}{l}\text { Simulation } \\
\text { Acceptance }\end{array}$ & $\begin{array}{l}\text { Percent } \\
\text { Difference }\end{array}$ & $\begin{array}{l}\text { Agreement } \\
\text { Factor }\end{array}$ \\
\hline 121.7817 & ${ }^{152} \mathrm{Eu}$ & $(3.108 \pm 0.004) \times 10^{-3}$ & $20.12 \%$ & 21.05 \\
244.6875 & ${ }^{152} \mathrm{Eu}$ & $(2.609 \pm 0.003) \times 10^{-3}$ & $4.83 \%$ & 4.86 \\
276.3997 & ${ }^{133} \mathrm{Ba}$ & $(2.494 \pm 0.003) \times 10^{-3}$ & $-1.28 \%$ & -0.98 \\
302.851 & ${ }^{133} \mathrm{Ba}$ & $(2.393 \pm 0.003) \times 10^{-3}$ & $-1.56 \%$ & -1.9 \\
344.2785 & ${ }^{152} \mathrm{Eu}$ & $(2.258 \pm 0.003) \times 10^{-3}$ & $4.06 \%$ & 6.23 \\
356.0134 & ${ }^{133} \mathrm{Ba}$ & $(2.227 \pm 0.003) \times 10^{-3}$ & $-0.81 \%$ & -1.97 \\
383.848 & ${ }^{133} \mathrm{Ba}$ & $(2.148 \pm 0.003) \times 10^{-3}$ & $-1.69 \%$ & -1.95 \\
411.1163 & ${ }^{152} \mathrm{Eu}$ & $(2.082 \pm 0.003) \times 10^{-3}$ & $-0.98 \%$ & -0.46 \\
443.965 & ${ }^{152} \mathrm{Eu}$ & $(2.007 \pm 0.003) \times 10^{-3}$ & $3.12 \%$ & 1.84 \\
778.904 & ${ }^{152} \mathrm{Eu}$ & $(1.564 \pm 0.003) \times 10^{-3}$ & $1.23 \%$ & 1.27 \\
867.373 & ${ }^{152} \mathrm{Eu}$ & $(1.490 \pm 0.003) \times 10^{-3}$ & $-1.02 \%$ & -0.71 \\
964.079 & ${ }^{152} \mathrm{Eu}$ & $(1.418 \pm 0.003) \times 10^{-3}$ & $0.86 \%$ & 1.09 \\
1112.069 & ${ }^{152} \mathrm{Eu}$ & $(1.329 \pm 0.003) \times 10^{-3}$ & $2.23 \%$ & 0.49 \\
1173.228 & ${ }^{60} \mathrm{Co}$ & $(1.326 \pm 0.003) \times 10^{-3}$ & $3.24 \%$ & 3.11 \\
1212.948 & ${ }^{152} \mathrm{Eu}$ & $(1.276 \pm 0.003) \times 10^{-3}$ & $1.32 \%$ & 0.47 \\
1274.537 & ${ }^{22} \mathrm{Na}$ & $(1.246 \pm 0.003) \times 10^{-3}$ & $-2.62 \%$ & -3.08 \\
1299.142 & ${ }^{152} \mathrm{Eu}$ & $(1.232 \pm 0.003) \times 10^{-3}$ & $-9.08 \%$ & -3.52 \\
1332.492 & ${ }^{60} \mathrm{Co}$ & $(1.215 \pm 0.003) \times 10^{-3}$ & $2.36 \%$ & 2.22 \\
1408.006 & ${ }^{152} \mathrm{Eu}$ & $(1.181 \pm 0.003) \times 10^{-3}$ & $0.35 \%$ & 0.45 \\
2341.2 & $\mu^{-} \mathrm{Au}$ & $(8.35 \pm 0.02) \times 10^{-4}$ & $-11.38 \%$ & -0.66 \\
2477.8 & $\mu^{-} \mathrm{Au}$ & $(8.07 \pm 0.02) \times 10^{-4}$ & $-10.5 \%$ & -0.51 \\
5591 & $\mu^{-} \mathrm{Au}$ & $(4.218 \pm 0.016) \times 10^{-4}$ & $0.99 \%$ & 0.05 \\
5763.1 & $\mu^{-} \mathrm{Au}$ & $(4.063 \pm 0.016) \times 10^{-4}$ & $5.52 \%$ & 0.16 \\
\hline
\end{tabular}




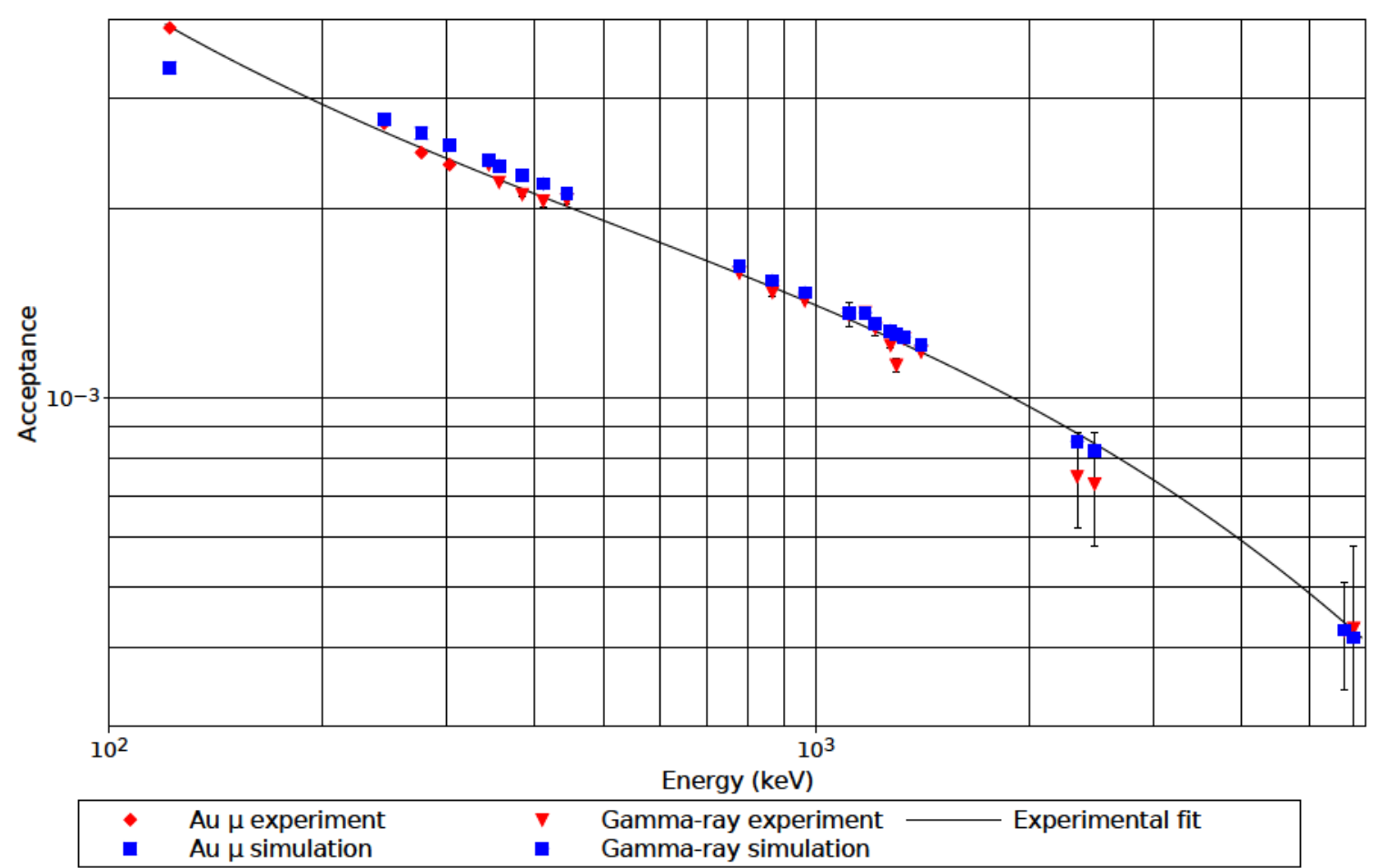

Figure 5.4: Experimental and simulated acceptances for the germanium detector. ${ }^{22} \mathrm{Na},{ }^{60} \mathrm{Co},{ }^{133} \mathrm{Ba},{ }^{152} \mathrm{Eu}$ were the gamma sources used for calibration, and $\mu^{-} \mathrm{x}$ rays from gold were used for the high energy calibration. No scintillator present in this simulation.

Table 5.10: Energy deposited into a phantom by one muon for a given energy at a given maximum path length.

\begin{tabular}{|l|l|l|l|}
\hline & \multicolumn{3}{|c|}{ Maximum path length $(\mathrm{cm})$} \\
\hline & 30 & 46 & 175 \\
\hline Energy $(\mathrm{keV})$ & \multicolumn{3}{|c|}{ Energy deposited $(\mathrm{keV})$} \\
\hline 1 & 1 & 1 & 1 \\
10 & 10 & 10 & 10 \\
100 & 100 & 100 & 100 \\
$1.0 \times 10^{3}$ & $1.0 \times 10^{3}$ & $1.0 \times 10^{3}$ & $1.0 \times 10^{3}$ \\
$1.0 \times 10^{4}$ & $1.0 \times 10^{4}$ & $1.0 \times 10^{4}$ & $1.0 \times 10^{4}$ \\
$3.0 \times 10^{4}$ & $3.0 \times 10^{4}$ & $3.0 \times 10^{4}$ & $3.0 \times 10^{4}$ \\
$1.0 \times 10^{5}$ & $1.0 \times 10^{5}$ & $1.0 \times 10^{5}$ & $1.0 \times 10^{5}$ \\
$1.0 \times 10^{6}$ & $7.1 \times 10^{5}$ & $1.0 \times 10^{6}$ & $4.0 \times 10^{6}$ \\
$1.0 \times 10^{7}$ & $9.2 \times 10^{5}$ & $1.4 \times 10^{6}$ & $5.3 \times 10^{6}$ \\
$1.0 \times 10^{8}$ & $1.1 \times 10^{6}$ & $1.7 \times 10^{6}$ & $6.7 \times 10^{6}$ \\
$1.0 \times 10^{9}$ & $1.3 \times 10^{6}$ & $2.1 \times 10^{6}$ & $8.0 \times 10^{6}$ \\
\hline
\end{tabular}


Table 5.11: The dose equivalent that one muon of a given energy at a maximum path length would deposit into a human of mass $70 \mathrm{~kg}$.

\begin{tabular}{|l|l|l|l|}
\hline & \multicolumn{3}{|c|}{ Maximum path length (cm) } \\
\hline & 30 & \multicolumn{3}{|c|}{46} & 175 \\
\hline Energy (keV) & \multicolumn{3}{|c|}{ Dose equivalent (Sv) } \\
\hline 1 & $2.2 \times 10^{-18}$ & $2.2 \times 10^{-18}$ & $2.2 \times 10^{-18}$ \\
10 & $2.2 \times 10^{-17}$ & $2.2 \times 10^{-17}$ & $2.2 \times 10^{-17}$ \\
100 & $2.2 \times 10^{-16}$ & $2.2 \times 10^{-16}$ & $2.2 \times 10^{-16}$ \\
$1.0 \times 10^{3}$ & $2.2 \times 10^{-15}$ & $2.2 \times 10^{-15}$ & $2.2 \times 10^{-15}$ \\
$1.0 \times 10^{4}$ & $2.2 \times 10^{-14}$ & $2.2 \times 10^{-14}$ & $2.2 \times 10^{-14}$ \\
$3.0 \times 10^{4}$ & $6.8 \times 10^{-14}$ & $6.8 \times 10^{-14}$ & $6.8 \times 10^{-14}$ \\
$1.0 \times 10^{5}$ & $2.2 \times 10^{-13}$ & $2.2 \times 10^{-13}$ & $2.2 \times 10^{-13}$ \\
$1.0 \times 10^{6}$ & $1.6 \times 10^{-13}$ & $2.4 \times 10^{-13}$ & $9.3 \times 10^{-13}$ \\
$1.0 \times 10^{7}$ & $2.1 \times 10^{-13}$ & $3.2 \times 10^{-13}$ & $1.2 \times 10^{-12}$ \\
$1.0 \times 10^{8}$ & $2.6 \times 10^{-13}$ & $4.0 \times 10^{-13}$ & $1.5 \times 10^{-12}$ \\
$1.0 \times 10^{9}$ & $3.1 \times 10^{-13}$ & $4.8 \times 10^{-13}$ & $1.8 \times 10^{-12}$ \\
\hline
\end{tabular}

Table 5.12: The number of muon stops of a given energy at a maximum path length required to attain a dose equivalent of $1 \mathrm{mSv}$ in the same human phantom.

\begin{tabular}{|l|l|l|l|}
\hline & \multicolumn{3}{|c|}{ Maximum path length $(\mathrm{cm})$} \\
\hline & 30 & \multicolumn{3}{|c|}{46} & 175 \\
\hline Energy $(\mathrm{keV})$ & \multicolumn{3}{|c|}{ Muons for one mSv } \\
\hline 1 & $4.4 \times 10^{14}$ & $4.4 \times 10^{14}$ & $4.4 \times 10^{14}$ \\
10 & $4.4 \times 10^{13}$ & $4.4 \times 10^{13}$ & $4.4 \times 10^{13}$ \\
100 & $4.4 \times 10^{12}$ & $4.4 \times 10^{12}$ & $4.4 \times 10^{12}$ \\
$1.0 \times 10^{3}$ & $4.4 \times 10^{11}$ & $4.4 \times 10^{11}$ & $4.4 \times 10^{11}$ \\
$1.0 \times 10^{4}$ & $4.4 \times 10^{10}$ & $4.4 \times 10^{10}$ & $4.4 \times 10^{10}$ \\
$3.0 \times 10^{4}$ & $1.4 \times 10^{11}$ & $1.4 \times 10^{11}$ & $1.4 \times 10^{11}$ \\
$1.0 \times 10^{5}$ & $4.4 \times 10^{9}$ & $4.4 \times 10^{9}$ & $4.4 \times 10^{9}$ \\
$1.0 \times 10^{6}$ & $6.1 \times 10^{10}$ & $4.0 \times 10^{10}$ & $1.0 \times 10^{10}$ \\
$1.0 \times 10^{7}$ & $4.7 \times 10^{10}$ & $3.0 \times 10^{10}$ & $8.1 \times 10^{9}$ \\
$1.0 \times 10^{8}$ & $3.8 \times 10^{10}$ & $2.4 \times 10^{10}$ & $6.5 \times 10^{9}$ \\
$1.0 \times 10^{9}$ & $3.1 \times 10^{10}$ & $2.0 \times 10^{10}$ & $5.4 \times 10^{9}$ \\
\hline
\end{tabular}




\section{Chapter 6}

\section{Discussion}

\subsection{Peak Identification}

Many different photons were identified, as seen in Table 5.3. These include muonic $\mathrm{x}$ rays from ${ }^{238} \mathrm{U}$ (Table 5.4), two candidate muonic $\mathrm{x}$ rays from fission products (Table 4.3), $\gamma$ rays from the ${ }^{238} \mathrm{U}$ decay chain, and neutron caused $(n, \gamma)$ events.

As mentioned in section 3.2.2, there was a $40 \mu$ s gate formed. However, the mean lifetime of a muon in uranium is $(77.0 \pm 0.4) \mathrm{ns}$. Thus, with most of the muon events completing within 3 to 5 lifetimes, there is still a great deal of time for ambient photons to enter the detector and deposit their energy.

Although some background lines were $(n, \gamma)$ lines, the introduction of the muon beam increases the number of neutrons produced, as muon capture can produce free neutrons.

Oftentimes, several gamma rays correspond with one another - that is to say that the presence of one should indicate the presence of another line. In Table 5.3,

there are some intensities that do not match up. These are primarily the ${ }^{234 m} \mathrm{~Pa}$ and ${ }^{234} \mathrm{~Pa}$ lines. The intensities for these lines are far above what the literature values suggest. In order to determine this, the ratio of intensities of the photopeaks of a 
given identification were compared with the literature values of those ratios. If the ratios were different by a significant amount, then the identifications were flagged as uncertain.

\subsection{1 $\quad{ }^{238} \mathrm{U} \alpha$ Decay}

Other $\gamma$ rays identified include several $\gamma$ rays from the radioactive decay of ${ }^{238} \mathrm{U} .{ }^{238} \mathrm{U}$ undergoes $\alpha$ decay, producing ${ }^{234} \mathrm{Th}$, which then further decays. The full decay chain can be seen in Figure 6.1. The $\gamma$ rays observed come from the nuclear de-excitation of the daughter nuclei. Most of these $\gamma$ rays were seen in both runs (with and without the muon beam), and so are not reported in Table 5.3.

There are some identifications in Table 5.3 consistent with these $\gamma$ rays. However, one would expect these to also be present in the background table (Table 4.1). Thus, it is possible that it is only by chance that these lines share the same energy with the decay chain $\gamma$ rays. It is also possible that due to the overly long gate for muons ( $40 \mu \mathrm{s})$, that more of these photons were available to be seen.

\subsection{2 $\mu^{-}$X Ray Candidates}

Muonic $\mathrm{x}$ rays were identified on uranium and candidate $\mathrm{x}$ rays were identified on two potential fission products, cesium and molybdenum, as seen in Table 5.5. It is possible that these are not in fact muonic $\mathrm{x}$ rays, and could simply be $\gamma$ rays related to the presence of muons. If these are muonic $\mathrm{x}$ rays, this would imply that these muonic $\mathrm{x}$ rays can be detected, and further would suggest that muonic $\mathrm{x}$ rays on fission products can be identified and detected. The cesium line has a rather large error on the area, which may be explained by the fact that two peaks are within $3 \mathrm{keV}$ of each other. Other fission product $\mu^{-} \mathrm{x}$ rays were searched for, but an upper limit was placed on these, as they were not distinguishable from the background. It 


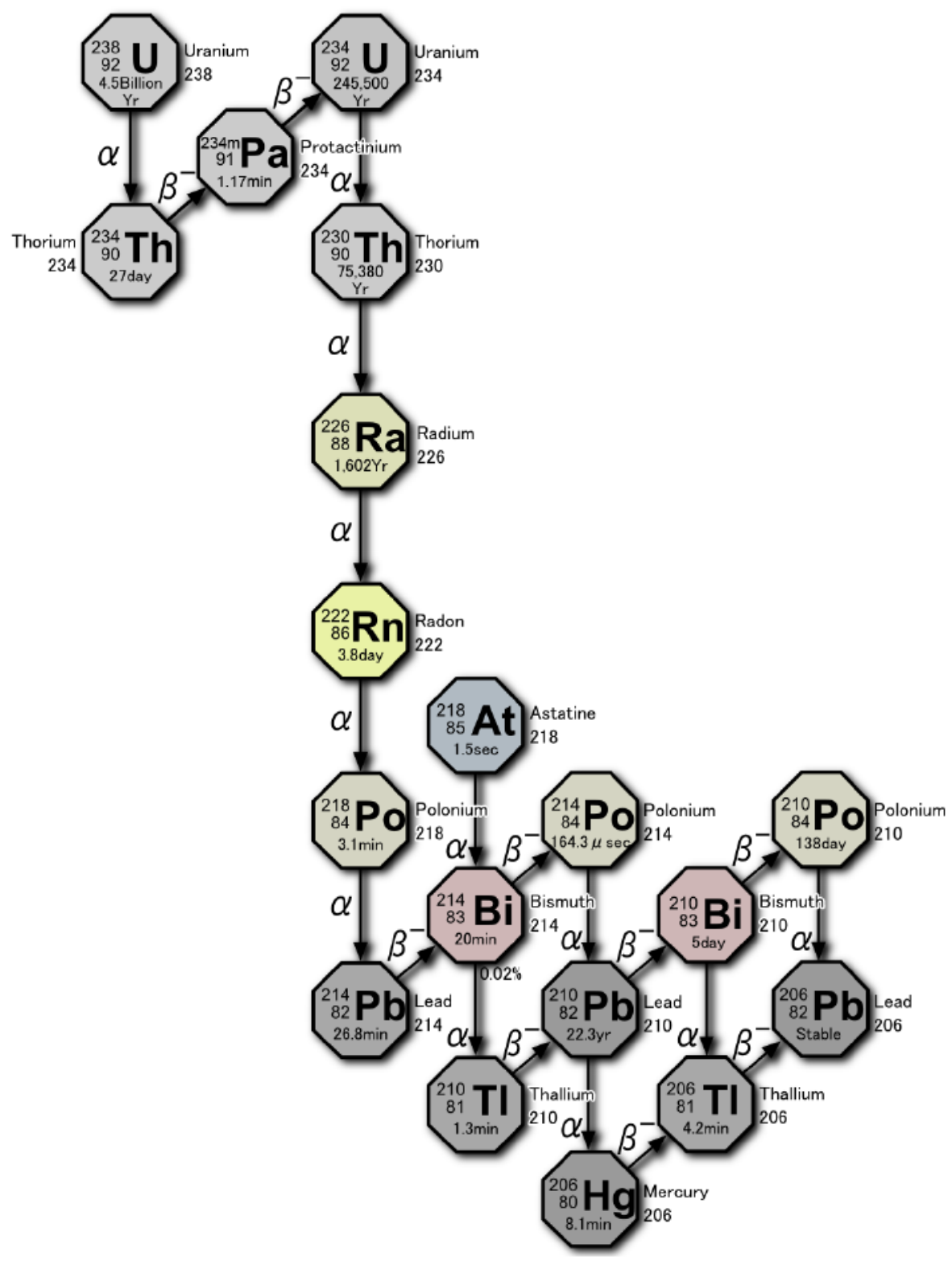

Figure 6.1: The ${ }^{238} \mathrm{U}$ decay chain. Image licensed under Creative Commons Attribution 3.0 from [46]. 
is possible that with a longer exposure time that these $\mathrm{x}$ rays can be successfully identified.

There are different processes for how these fission product muonic $\mathrm{x}$ rays could have been produced. The first is that the uranium undergoes fission - either spontaneous or muon induced as discussed in section 1.10 - and produces molybdenum and cesium (among other fission products) in the target. Another muon is then atomically captured on these daughter nuclei, and undergoes a muonic cascade, resulting in the muonic $\mathrm{x}$ rays seen in the spectrum. For this event, the expected number of counts is given by rearranging equation (3.3) to get

$$
A_{f p}=\frac{\xi_{f p} S_{f p} L E_{p, f p}}{f_{s a, f p}}
$$

where $A_{f p}$ is the number of counts in the fission product line, $\xi_{f p}$ is the acceptance of the fission product line, $S_{f p}$ is the number of muon stops in the fission product, $L$ is the live time, $E_{p, f p}$ is the emission probability of the fission product x ray, and $f_{s a, f p}$ is the self-absorption coefficient of the fission product.

In order to obtain an estimate of how many muon stops in the fission product, $S_{f p}$, we take the number of muon stops in uranium, $S_{U}$, and multiply by the ratio of atomic capture rates, $p$.

$$
S_{f p}=S_{U} \cdot p
$$

By rearranging equation (3.3), we have that the number of muon stops in uranium is given by

$$
S_{U}=\frac{A_{U} f_{s a, U}}{\xi_{U} L E_{p, U}}
$$

where the subscript $U$ refers to the uranium line.

The ratio of atomic capture rates is approximately given by $p=Z_{f p} N_{f p} / Z_{U} N_{U}$ (although this can be off by a factor of 2 or more), where $Z_{f p}$ and $Z_{U}$ are the atomic number of the fission product and the uranium respectively, and $N_{f p}$ and $N_{U}$ are the number of fission product atoms and uranium atoms respectively [17]. This is needed 
to obtain the number of muon stops. To obtain an estimate of how many of the daughter nuclei are in the sample, $N_{f p}$, the available data on the uranium source can be examined. The uranium source was measured to have an activity of $6.00 \times 10^{2} \mathrm{~Bq}$ on October 19, $1995\left(=t_{0}\right)$, and the experiment was performed on August 14, 2009. Thus we get $t=5048$ days $=4.361 \times 10^{8} \mathrm{~s}$. Without knowing the actual age of the source or the last time any impurities were removed, it is assumed that on that date, it was pure ${ }^{238} \mathrm{U}$, and has not had any impurities removed since. It is also assumed that any fission products resulting from muon induced fission are negligible, since the total exposure to muons is not known. Thus, if $N_{U, 0}$ is the number of uranium atoms at time $t_{0}, \lambda$ is the half-life of uranium, the radioactive decay equation gives us

$$
N_{U}(t)=N_{U, 0} e^{-\lambda t}
$$

Then the total number of uranium decay products at will be

$$
\begin{aligned}
N_{\text {decay }} & =N_{U, 0}-N_{U}(t) \\
& =N_{U, 0}\left(1-e^{-\lambda t}\right)
\end{aligned}
$$

The number that will be spontaneous fission (SF) products is obtained by multiplying equation (6.5) by the SF yield, which for ${ }^{238} \mathrm{U}$ is $B=5.45 \times 10^{-5} \%$. Finally, the number of specific decay products is obtained by multiplying this by the fissile yield $\left(y_{f}\right)$ of ${ }^{238} \mathrm{U}$ for that product, seen in Table 4.2. This gets us

$$
N_{f p}(t)=B y_{f} N_{U, 0}\left(1-e^{-\lambda t}\right)
$$

Combining this with equation (6.4), and dividing by $N_{U}(t)$ to match up with the atomic capture rate ratio of $N_{f p} / N_{U}$, we get

$$
\frac{N_{f p}(t)}{N_{U}(t)}=B y_{f} \frac{1-e^{-\lambda t}}{e^{-\lambda t}}
$$


Table 6.1: Predicted number of counts from the "existing fission product" method of obtaining muonic $\mathrm{x}$ rays from fission products.

\begin{tabular}{|c|c|}
\hline Candidate & Predicted Number of Counts \\
\hline${ }^{98} \mathrm{Mo}$ & $5.0 \times 10^{-13}$ \\
${ }^{133} \mathrm{Cs}$ & $1.2 \times 10^{-12}$ \\
\hline
\end{tabular}

This gives us a final equation for the estimate of how many events we should see for the candidate products

$$
\begin{aligned}
A_{f p} & =\frac{\xi_{f p} L E_{p, f p}}{f_{s a, f p}} \frac{A_{U} f_{s a, U}}{\xi_{U} L E_{p, U}} \frac{Z_{f p}}{Z_{U}} B y_{f} \frac{1-e^{-\lambda t}}{e^{-\lambda t}} \\
& =A_{U} \frac{\xi_{f p}}{\xi_{U}} \frac{E_{p, f p}}{E_{p, U}} \frac{f_{s a, U}}{f_{s a, f p}} \frac{Z_{f p}}{Z_{U}} B y_{f} \frac{1-e^{-\lambda t}}{e^{-\lambda t}}
\end{aligned}
$$

We then have that $f_{s a, f p} \approx f_{s a, U}$, and so we treat that factor as 1 . Since the value $E_{p, f p}$ is not known, we take it to be 1 , and place an upper limit on the estimated number of counts.

$$
A_{f p} \approx \frac{A_{U}}{E_{p, U}} \frac{\xi_{f p}}{\xi_{U}} \frac{Z_{f p}}{Z_{U}} B y_{f} \frac{1-e^{-\lambda t}}{e^{-\lambda t}}
$$

This leads to the expected photopeak area seen in Table 6.1. As can be seen, the number of counts is $<1$ in both cases, and thus, unless there is considerably more of the daughter nuclei in the target than estimated, and this is likely not how these candidates were formed.

The second process starts when a muon is atomically captured on uranium. Then, either the nucleus undergoes spontaneous fission or the muon undergoes a nonradiative transition inducing fission, and the muon transfers to the daughter nuclei (typically the heavier of the two $[47,48])$. At this point, the muon then undergoes a muonic cascade, producing the muonic x rays. As before, we start with equation (6.1). This time, the number of muon stops is given by

$$
S_{f p}=S_{U} \cdot p_{f} y_{f}
$$


Table 6.2: Predicted number of counts from the "muon transfer" method of obtaining muonic $\mathrm{x}$ rays from fission products.

\begin{tabular}{|c|c|}
\hline Candidate & Predicted Number of Counts \\
\hline${ }^{98} \mathrm{Mo}$ & $70 \pm 30$ \\
${ }^{133} \mathrm{Cs}$ & $120 \pm 50$ \\
\hline
\end{tabular}

where $p_{f}$ is the probability of the muon undergoing fission and $y_{f}$ is the yield for that particular fission product from Table 4.2. Using equation (6.3) again, combine these equations to get

$$
\begin{aligned}
A_{f p} & =\frac{\xi_{f p} L E_{p, f p}}{f_{s a, f p}} \frac{A_{U} f_{s a, U}}{\xi_{U} L E_{p, U}} p_{f} y_{f} \\
& \approx \frac{A_{U}}{E_{p, U}} \frac{\xi_{f p}}{\xi_{U}} p_{f} y_{f}
\end{aligned}
$$

As before, we take $f_{s a, f p} \approx f_{s a, U}$ and $E_{p, f p} \approx 1$ to get an upper limit. Taking $p_{f}=(7.5 \pm 1.0) \%$ [21], we get the results seen in Table 6.2. The predicted area from this methods falls 1 order of magnitude short for the ${ }^{98} \mathrm{Mo}$ candidate, but is above the number of counts for the ${ }^{133} \mathrm{Cs}$ candidate.

\subsubsection{Yield}

The $\mathrm{x}$ ray yield is how often the $\mathrm{x}$ ray is emitted per muon stop in the target. We can calculate this for the two fission product candidate $\mathrm{x}$ rays with equation (6.12). However, the number of muon stops $(S)$ is not known (since a third scintillator was not used). By using the yield calculated in another paper [1] using these same data for a particular line, we can calculate the number of muon stops with equation (6.13). To minimize error, we use the $(6119.0 \pm 1.6) \mathrm{keV}$ line, with a yield of $0.152 \pm 0.055$.

$$
\begin{gathered}
y_{\text {candidate }}=\frac{A_{\text {candidate }} f_{s a}}{S L \xi_{\text {candidate }}} \\
S=\frac{A_{U} f_{s a}}{L \xi_{U} y_{U}}
\end{gathered}
$$


Note that for calculating the yield of the cesium and molybdenum muonic $\mathrm{x}$ ray candidates, we do not use the self-absorption coefficent for cesium or molybdenum, but instead that of uranium. This is because the $\mathrm{x}$ rays will almost exclusively be traveling through uranium. In particular, since the mass attenuation coefficients for uranium at the three different energies are almost the same, we can say that $f_{s a} \approx$ constant for those three energies. Combining equations (6.12) and (6.13), we get

$$
\begin{aligned}
y_{\text {candidate }} & =\frac{A_{\text {candidate }} f_{s a}}{L \xi_{\text {candidate }}} \frac{L \xi_{U} y_{U}}{A_{U} f_{s a}} \\
& =y_{\mathrm{U}} \frac{A_{\text {candidate }}}{A_{\mathrm{U}}} \frac{\xi_{\mathrm{U}}}{\xi_{\text {candidate }}}
\end{aligned}
$$

This leads to the yields found in Table 6.3.

Table 6.3: Yield (x ray transitions per muon stop) for the muonic $2 p \rightarrow 1 s$ x-ray candidates.

\begin{tabular}{|c|c|c|c|}
\hline Identification & $\begin{array}{c}\text { Energy } \\
(\mathrm{keV})\end{array}$ & Number of Counts & $\begin{array}{c}\text { Yield } \\
\text { (x ray transitions per muon stop) }\end{array}$ \\
\hline${ }^{98} \mathrm{Mo}$ & $2378.0 \pm 0.7$ & $(1.10 \pm 0.15) \times 10^{3}$ & $0.06 \pm 0.02$ \\
${ }^{133} \mathrm{Cs}$ & $3900.2 \pm 1.7$ & $50 \pm 50$ & $0.04 \pm 0.04$ \\
\hline
\end{tabular}

\subsection{Nuclear Forensics}

As discussed in section 1.1, one is interested in calculating the relative ratio of different isotopes of uranium. Since the uranium target was only depleted uranium (exclusively ${ }^{238} \mathrm{U}$ ), this cannot be calculated. However, by calculating the minimum detectable activity (MDA) of the peak of interest, we can obtain insight into the sensitivity of the technique. To do this, we need to calculate the detection limit, given by equation (6.15) [19], where $L_{D}$ is the detection limit, $\mathrm{B}$ is the number of counts in the background, $n$ is the number of channels in the peak, and $m$ is the number of channels used to calculate the background on either side of the peak, and 2.71 and 3.29 are 
constants obtained from using a $95 \%$ confidence interval. Equation (6.15) is obtained by examining the minimum number of counts one can be confident of detecting, and is based on the number of counts in the background, as well as the spread in that number. We then convert this limit to an activity by using equation (6.16), where $\xi$ is the acceptance at the peak energy. For the $(6119.0 \pm 1.6) \mathrm{keV}$ line, this gives us $L_{D}=167$ and $M D A=4.29 \times 10^{5} \mathrm{X}$ ray transitions per second.

$$
\begin{gathered}
L_{D}=2.71+3.29 \sqrt{B\left(1+\frac{n}{2 m}\right)} \\
M D A=\frac{L_{D}}{\xi}
\end{gathered}
$$

This method would allow us to calculate the relative activity of different isotopes, as the energy spectrum for different isotopes differs, as can be seen in Figure 1.1. Since nuclear forensics aims to identify the source of a sample of SNM, several techniques examine either the isotopic content of the sample $[49,50,51]$, whereas others examine the ratios of isotopes within the sample [52, 53, 54]. Consider SF-ICP-MS (Sector Field Inductively Coupled Plasma Mass Spectrometry) or MC-ICP-MS (Multiple Collector ICP-MS). These are destructive techniques, that take a portion of the sample material and examine the ratio between isotopes of uranium, typically ${ }^{238} \mathrm{U} /{ }^{235} \mathrm{U},{ }^{236} \mathrm{U} /{ }^{238} \mathrm{U}$, and ${ }^{234} \mathrm{U} /{ }^{238} \mathrm{U}$. These two techniques can determine these ratios to within $0.046 \%$ to $3.0 \%$ [53]. However, since no muonic $\mathrm{x}$ rays from any of these isotopes were identified, a comparison between this technique and others cannot be directly made.

\subsection{Acceptances}

The acceptances of the detector were simulated using the EGSnrc Monte Carlo toolkit. These simulations were done to verify the acceptance calculations, as well as the 
calculation for the number of muon stops. This was also done because another member of the group attempted to simulate these acceptances in MCNPX, but obtained values 1.5 to 2 times above the measured accepted values. The simulation was run with $1.3 \times 10^{6}$ events, and led to statistical errors of less than $1 \%$. The RANLUX random number generator has a period of approximately $10^{117}$, and thus even at the low luxury level ${ }^{1}$ of 1 , was still able to handle the simulation without any repetition on the period.

Four different simulations were run, at four different thicknesses of the plastic electron veto scintillator: $0 \mathrm{~cm}, 1 / 16 \mathrm{inch}, 1 / 8 \mathrm{inch}$, and $0.5 \mathrm{~cm}$. Of the 23 peaks used, one of them (the $121.7817 \mathrm{keV}$ line from ${ }^{152} \mathrm{Eu}$ ) had a simulated acceptance well below the accepted (calculated) value. Specifically, it ranged from $14.5 \%$ to $22.4 \%$ below the measured accepted value. Because we are most interested in the acceptance at higher energies, this data point was removed from consideration.

In Table 6.4, the total number of sigma difference can be seen. The absolute value is minimized for the $5 \mathrm{~mm}$ scintillator, agreeing with the speculation that the actual thickness of the scintillator was $5 \mathrm{~mm}$.

The acceptances being successfully simulated lends credibility to both the original calculation performed by Olsthoorn, as well as the validity of using EGSnrc to simulate detector acceptances. This could be used to estimate the acceptance of a more complicated detector-source geometry and determine if it's possible to detect these photopeaks in such a geometry.

\subsection{Dose from Muons}

The dose equivalent deposited by a single muon at various energies was calculated, as was the number of muons it would take to reach a total dose equivalent of $1 \mathrm{mSv}$.

\footnotetext{
${ }^{1}$ The luxury level is a number indicating the tradeoff between high randomness and computation time. A higher luxury level indicates that the computation will take longer, but will be more random.
} 
Table 6.4: The sum of the number of sigma difference for all four scintillator thicknesses.

\begin{tabular}{|c|c|}
\hline Scintillator thickness & Sum of number of sigma difference \\
\hline $0 \mathrm{~cm}$ & -72 \\
$0.15875 \mathrm{~cm}$ & -48 \\
$0.3175 \mathrm{~cm}$ & -25 \\
$0.5 \mathrm{~cm}$ & 6.5 \\
\hline
\end{tabular}

The value of $1 \mathrm{mSv}$ was chosen because this is the limit that the Radiation Protection Regulations sets out for a person who is not a nuclear energy worker [5] for a period of one year. One of the original ideas when this research was proposed was that this method could be used for standoff detection (detection at a distance) of SNM.

It is worth noting that the calculation done was very simplistic. Thus, a more complete simulation would need to be done in order to properly estimate the dose equivalent rate. A simulation toolkit such as GEANT4 would be appropriate for this purpose.

Previous work by Siiskonen [24] concluded that for slow muons (i.e. muons likely to stop in the material), the radiation weighting factor is greater than unity, at least for skin exposure. Neutrons and gamma rays are produced in every muon capture, and protons and alpha particles can be produced as well, especially in light nuclei. It is predominantly these protons and alpha particles that increase the weighting factor above unity.

Knowing the dose equivalent provided by a muon sets a limit on the beam time and intensity that can be used to scan things (i.e. how many muons can be fired at the target). Combined with the acceptance, one can thus find a limit on the maximum intensity one can obtain. For example, if one is using active muon interrogation for stand-off detection, there is an upper limit on the number of muons that can be used for investigation, and this places an upper limit on the intensities of the signals. 


\section{Chapter 7}

\section{Conclusions and Future Work}

Gamma-ray spectrometry was used to search for and identify several peaks from a muon beam hitting a target of ${ }^{238} \mathrm{U}$. Several muonic $\mathrm{x}$ rays were identified. Several photopeaks remain unidentified. Future work can be done to obtain identifications for these peaks.

Two candidates for muonic $\mathrm{x}$ rays occurring from fission products were also identified. The first was the $2 p \rightarrow 1 s \mu^{-}$x-ray from ${ }^{98} \mathrm{Mo}$ at $(2738.0 \pm 0.7) \mathrm{keV}$ with a yield of $(0.06 \pm 0.02) \mathrm{x}$ ray transitions per muon stop. The second was the $2 p \rightarrow 1 s$ $\mu^{-} \mathrm{x}$-ray from ${ }^{133} \mathrm{Cs}$ at $(3900.2 \pm 1.7) \mathrm{keV}$ with a yield of $0.04 \pm 0.04 \mathrm{x}$ ray transitions per muon stop. Estimates of the number of counts implies that the number of counts actually seen for the ${ }^{98}$ Mo candidate is higher than expected. Future work can be done to see if these fission products are actually present in the target, and if these peaks can be more readily identified. More work can also be done to improve the statistics on these peaks, especially the ${ }^{133} \mathrm{Cs}$ candidate.

The acceptance simulations yielded acceptances similar to those calculated by Olsthoorn. However, since EGSnrc only supports electrons, positrons, and gammas, an improved simulation involving the actual muon beam could be performed. A possible candidate would be the GEANT4 (GEometry ANd Tracking) Monte Carlo 
simulation toolkit. It would allow for the simulation of muons and muon physics, and allow for the simulation of more of the experimental setup, as opposed to just the target and detector.

The dose equivalent from muons was also calculated using a simulation and the Bethe-Bloch equation. An upper limit of $1.4 \times 10^{11}$ muons was calculated as the maximum number of muon stops required to hit a dose equivalent limit of $1 \mathrm{mSv}$ in a human phantom. The simulation used was a very simplified model, ignoring several processes such as scattering, ionization, and other effects. A better simulation could be written in a more powerful MC tool such as EGSnrc for the purposes of better calculating the dose equivalent that muons deposit in the human body. This would improve safety should a system using this technique ever be developed.

A minimum detectable transition rate of $4.29 \times 10^{5} \mathrm{x}$-ray transitions per second was calculated for the strongest $\mu^{-}{ }^{238} \mathrm{U} \times$ ray. Should any ${ }^{235} \mathrm{U}$ be present in the sample, the ratio between the two could be determined, which could then be compared against the possible sources of the sample [6]. 


\section{References}

[1] T J Stocki, A Jason, H Miyadera, E I Esch, N J Hoteling, A Adelmann, R H Heffner, A Green, and J Olsthoorn. Using exotic atoms to keep borders safe. Journal of Physics: Conference Series, 312(6):062009, 2011.

[2] Andrew J. Jason, Haruo Miyadera, and Peter J. Turchi. SNM Detection by Active Muon Interrogation. In Proceedings of the Institute of Nuclear Materials Management, pages 1-10, January 2010.

[3] Haruo Miyadera. Private communication.

[4] Jason Olsthoorn. Muon interaction with natural chlorine. Cooperative education report, Health Canada, 2010.

[5] Government of Canada. Radiation protection regulations, September 2007. Retrieved from http://laws-lois.justice.gc.ca/eng/regulations/ SOR-2000-203/page-4.html.

[6] K Mayer, M Wallenius, K Ltzenkirchen, J Galy, Z Varga, N Erdmann, R Buda, J-V Kratz, N Trautmann, and K Fifield. Nuclear forensics: A methodology applicable to nuclear security and to non-proliferation. Journal of Physics: Conference Series, 312(6):062003, 2011. 
[7] D. A. Close, J. J. Malanify, and J. P. Davidson. Nuclear charge distributions deduced from the muonic atoms of ${ }^{232} \mathrm{Th},{ }^{235} \mathrm{U},{ }^{238} \mathrm{U}$, and ${ }^{239} \mathrm{Pu}$. Phys. Rev. $C$, 17(4):1433-1455, April 1978.

[8] Andrew Jason. Private communication.

[9] Harold Elford Johns and John Robert Cunningham. The Physics of Radiology. Charles C. Thomas, Springfield, Illinois, 4th edition, 1983.

[10] Hideki Yukawa. On the interaction of elementary particles. i. Proceedings of the Physico-Mathematical Society of Japan. 3rd Series, 17:48-57, 1935.

[11] Seth H. Neddermeyer and Carl D. Anderson. Note on the nature of cosmic-ray particles. Phys. Rev., 51(10):884-886, May 1937.

[12] J. C. Street and E. C. Stevenson. New evidence for the existence of a particle of mass intermediate between the proton and electron. Phys. Rev., 52(9):1003-1004, November 1937.

[13] C. M. G. Lattes, G. P. S. Occhialini, and C. F. Powell. Observations on the tracks of slow mesons in photographic emulsions. Nature, 160:453-459, October 1947.

[14] J. Beringer et. al. (Particle Data Group). 2013 Review of Particle Physics. Phys. Rev. D, 86(010001), 2012.

[15] M. Conversi, E. Pancini, and O. Piccioni. On the disintegration of negative mesons. Phys. Rev., 71(3):209-210, February 1947.

[16] W.Y. Chang. American Physical Society: Minutes of the 1948 Annual Meeting at New York, January 26-29, 1949. Phys. Rev., 75(8):1279-1336, April 1949.

[17] D.F. Measday. The nuclear physics of muon capture. Physics Reports, 354:243409, 2001. 
[18] R. Engfer, H. Schneuwly, J. L. Vuilleumier, H. K. Walter, and A. Zehnder. Charge-distribution parameters, isotope shifts, isomer shifts, and magnetic hyperfine constants from muonic atoms. Atomic Data and Nuclear Data Tables, 14(5,6):509-597, November/December 1974.

[19] Gordon Gilmore. Practical gamma-ray spectrometry. Wiley, Mississauga, 2008.

[20] N. E. Holden and D. C. Hoffman. Spontaneous fission half-lives for ground-state nuclides (technical report). Pure Appl.Chem., 72:1525-1562, 2000.

[21] Salahuddin Ahmad. Muon Induced Fission in ${ }^{235} \mathrm{U}$ and ${ }^{238} \mathrm{U}$. PhD thesis, University of Victoria, August 1981.

[22] V. Anghel, J. Armitage, J. Botte, K. Boudjemline, D. Bryman, E. Charles, T. Cousins, A. Erlandson, G. Gallant, C. Jewett, G. Jonkmans, Z. Liu, S. Noel, G. Oakham, T.J. Stocki, M. Thompson, and D. Waller. Cosmic ray muon tomography system using drift chambers for the detection of special nuclear materials. In Nuclear Science Symposium Conference Record (NSS/MIC), 2010 IEEE, pages 547-551, October 2010.

[23] ICRP. The 2007 Recommendations of the International Commission on Radiological Protection. Annals of the ICRP, 37(2-4), 2007.

[24] T. Siiskonen. Dose from slow negative muons. Radiation Protection Dosimetry, 128(2):234-238, 2008.

[25] Trevor John Stocki. Measurement Of Muonic Hyperfine Transition Rates and Muon Capture Yields in Light Nuclei. PhD thesis, The University of British Colombia, September 1998.

[26] T. J. Stocki, D. F. Measday, E. Gete, M. A. Saliba, J. Lange, and T. P. Gorringe. Hyperfine transition in light muonic atoms of odd Z. Phys. Rev. A, 64(4):042505, September 2001. 
[27] T.J. Stocki, D.F. Measday, E. Gete, M.A. Saliba, B.A. Moftah, and T.P. Gorringe. Gamma rays from muon capture in ${ }^{14} \mathrm{~N}$. Nuclear Physics A, 697(12):55-74, January 2002.

[28] Glen M. Marshall. Muon Beams at TRIUMF. Workshop on Instrumentation for Muon Cooling Studies at Blackett Laboratory, Imperial College, February 2001.

[29] M. Marchetto, R. Baartman, Yu-Chiu Chao, G. Soh, S.R. Koscielniak, R.E. Laxdal, and Fang Yun. Beam Dynamics Optimization of the TRIUMF eLinac Injector. In Proceedings of PAC09, pages 3937-3939.

[30] Marcello Pavan. Private communication.

[31] Rene Brun and Fons Rademakers. ROOT - An Object Oriented Data Analysis Framework. In Proceedings AIHENP'96 Workshop Nucl. Inst. 83 Meth. in Phys. Res. A, 389, pages 81-86, Lausanne, September 1997. See also http://root. cern.ch/.

[32] F.J. Hartmann, R. Bergmann, H. Daniel, H.-J. Pfeiffer, T. Egidy, and W. Wilhelm. Measurement of the muonic x-ray cascade in $\mathrm{Mg}, \mathrm{Al}, \mathrm{In}, \mathrm{Ho}$, and $\mathrm{Au}$. Zeitschrift für Physik A Atoms and Nuclei, 305(3):189-204, 1982.

[33] R. A. Meyer and T. N. Massey. Multigamma-ray calibration sources. The International Journal of Applied Radiation and Isotopes, 34(8):1073-1084, August 1983.

[34] Jim Fitzgerald. Fitzpeaks gamma analysis and calibration software. http:// www.jimfitz.demon.co.uk/fitzpeak.htm, June 2009. Retrieved Nov. 2013.

[35] Klaus Debertin and Richard G. Helmer. Gamma- and X-ray spectrometry with semiconductor detectors. Physical Sciences and Engineering Division, Elsevier Science Publishers, Amsterdam, 1988. 
[36] Winfrith Improved Multigroup Scheme. Fission product yields. https:// www-nds.iaea.org/wimsd/fpyield.htm. Retrieved Nov. 2013.

[37] Lindsay Beaton, An Tran, and Katerine Bock. Previous project work (Carleton University co-operative education report), 2005, 2006, and 2006 respectively.

[38] R. J. Powers, P. Martin, G. H. Miller, R. E. Welsh, and D. A. Jenkins. Muonic ${ }^{197} \mathrm{Au}$ : A test of the weak-coupling model. Nuclear Physics A, 230(3):413-444, February 1974.

[39] J. F. Ziegler. The stopping of energetic light ions in elemental matter. Applied Physics Reviews / Journal of Applied Physics, 85:1249-1272, 1999.

[40] National Nuclear Data Center Database. Radiation decay. Data retrieved from NNDC, http://www.nndc.bnl.gov/, 2014.

[41] D. Kameda, T. Kubo, et al. Observation of new microsecond isomers among fission products from in-flight fission of $345 \mathrm{mev} /$ nucleon ${ }^{238} \mathrm{U}$. Phys. Rev. C, 86(5):054319, November 2012.

[42] C. M. Folden, A. S. Nettleton, et al. New neutron-rich microsecond isomers observed among fission products of ${ }^{238} \mathrm{U}$ at $80 \mathrm{mev} / \mathrm{nucleon}$. Phys. Rev. C, 79(6):064318, June 2009.

[43] A.A. Hecht, R.E. Blakeley, W.J. Martin, and E. Leonard. Comparison of GEANT4 and MCNP6 for use in delayed fission radiation simulation. Annals of Nuclear Energy, 69:134-138, 2014.

[44] S. L. Hammond, A. S. Adekola, C. T. Angell, H. J. Karwowski, E. Kwan, G. Rusev, A. P. Tonchev, W. Tornow, C. R. Howell, and J. H. Kelley. Dipole response of ${ }^{238} \mathrm{U}$ to polarized photons below the neutron separation energy. Phys. Rev. C, 85(4):044302, April 2012. 
[45] Sylvian Kahane and Alex Wolf. Photofission of ${ }^{238} \mathrm{U}$ with neutron-capture gamma rays. Phys. Rev. C, 32(6):1944-1955, December 1985.

[46] Tosaka (Wikipedia author). Decay chain(4n+2, uranium series). http://en.wikipedia.org/wiki/File:Decay_chain\%284n\%2B2,_Uranium_ series\%29. PNG, December 2012.

[47] Dz. Ganzorig, P.G. Hansen, T. Johansson, B. Jonson, J. Konijn, T. Krogulski, V.D. Kuznetsov, S.M. Polikanov, G. Tibell, and L. Westgaard. Fission of muonic ${ }^{232} \mathrm{Th}$ and ${ }^{238} \mathrm{U}$. Physics Letters B, 77(3):257-261, 1978.

[48] W. U. Schröder, W. W. Wilcke, M. W. Johnson, D. Hilscher, J. R. Huizenga, J. C. Browne, and D. G. Perry. Evidence for atomic muon capture by fragments from prompt fission of muonic ${ }^{237} \mathrm{~Np},{ }^{239} \mathrm{Pu}$, and ${ }^{242} \mathrm{Pu}$. Phys. Rev. Lett., 43(10):672675, September 1979.

[49] Zsolt Stefanka, Robert Katona, and Zsolt Varga. Laser ablation assisted icp-ms as a tool for rapid categorization of seized uranium oxide materials based on isotopic composition determination. J. Anal. At. Spectrom., 23(7):1030-1033, 2008.

[50] G. Nicolaou. Determination of the origin of unknown irradiated nuclear fuel. Journal of Environmental Radioactivity, 86(3):313-318, 2006.

[51] M. Wallenius, K. Mayer, and I. Ray. Nuclear forensic investigations: two case studies. Forensic Science International, 156(1):52-62, January 2006.

[52] Zsolt Varga and Gergely Surnyi. Detection of previous neutron irradiation and reprocessing of uranium materials for nuclear forensic purposes. Applied Radiation and Isotopes, 67(4):516-522, April 2009.

[53] Randall R. Parrish, Matthew F. Thirwall, Chris Pickford, Matthew Horstwood, Axel Gerdes, James Anderson, and David Coggon. Determination of ${ }^{238} \mathrm{U} /{ }^{235} \mathrm{U}$, 
${ }^{236} \mathrm{U} /{ }^{238} \mathrm{U}$ and uranium concentration in urine using SF-ICP-MS and MC-ICPMS: an interlaboratory comparison. Health Physics, 90(2):127-138, February 2006.

[54] Klaus Mayer, Maria Wallenius, and Ian Ray. Nuclear forensics - a methodology providing clues on the origin of illicitly trafficked nuclear materials. Analyst, 130(4):433-441, 2005.

[55] D.W.O. Rogers, I. Kawrakow, J.P. Seuntjens, B.R.B. Walters, and E. MainegraHing. NRC User Codes for EGSnrc. National Research Council Canada, February 2010 . 


\section{Appendix A}

\section{Sample EGS Input File}

Below is the .egsinp file used for the ${ }^{22} \mathrm{Na}$ acceptance simulation. Similar files were used for simulating other photopeaks. This includes the geometry required to simulate a point source impinging upon the detector. Information about how to read an .egsinp file can be found in the user manual for EGSnrc [55].

The bin numbers found below were chosen empirically by Beaton [37].

For most of the options in the file below, the default is sufficient, but some need to be changed if the geometry is modified:

- INCIDENT KINETIC ENERGY(MEV) must be set to the appropriate energy.

- SOURCE OPTIONS must be updated if the source distance is changed, or if the source type (SOURCE NUMBER) is changed from a point source.

For the I/O control section, special care must be taken with the DOSE ZBOUND MIN/MAX and DOSE RBOUND MIN/MAX settings. If any extra layers are added to the simulation (say for the simulation of the electron veto scintillator), these must be added to the MAX settings. The DEPTH BOUNDARIES and RADII settings in the geometrical inputs section are where the geometrical details belong. Note that the boundaries are defined as cumulative values, not as delta values. When changing the boundaries, 
care must be taken when defining the media, as the ZSLAB and RING values will shift. Finally, if the regions are changed, the REGION OF SENSITIVE VOLUME setting must be adjusted as well. How to find out which volumes are sensitive can be found in the user manual [55].

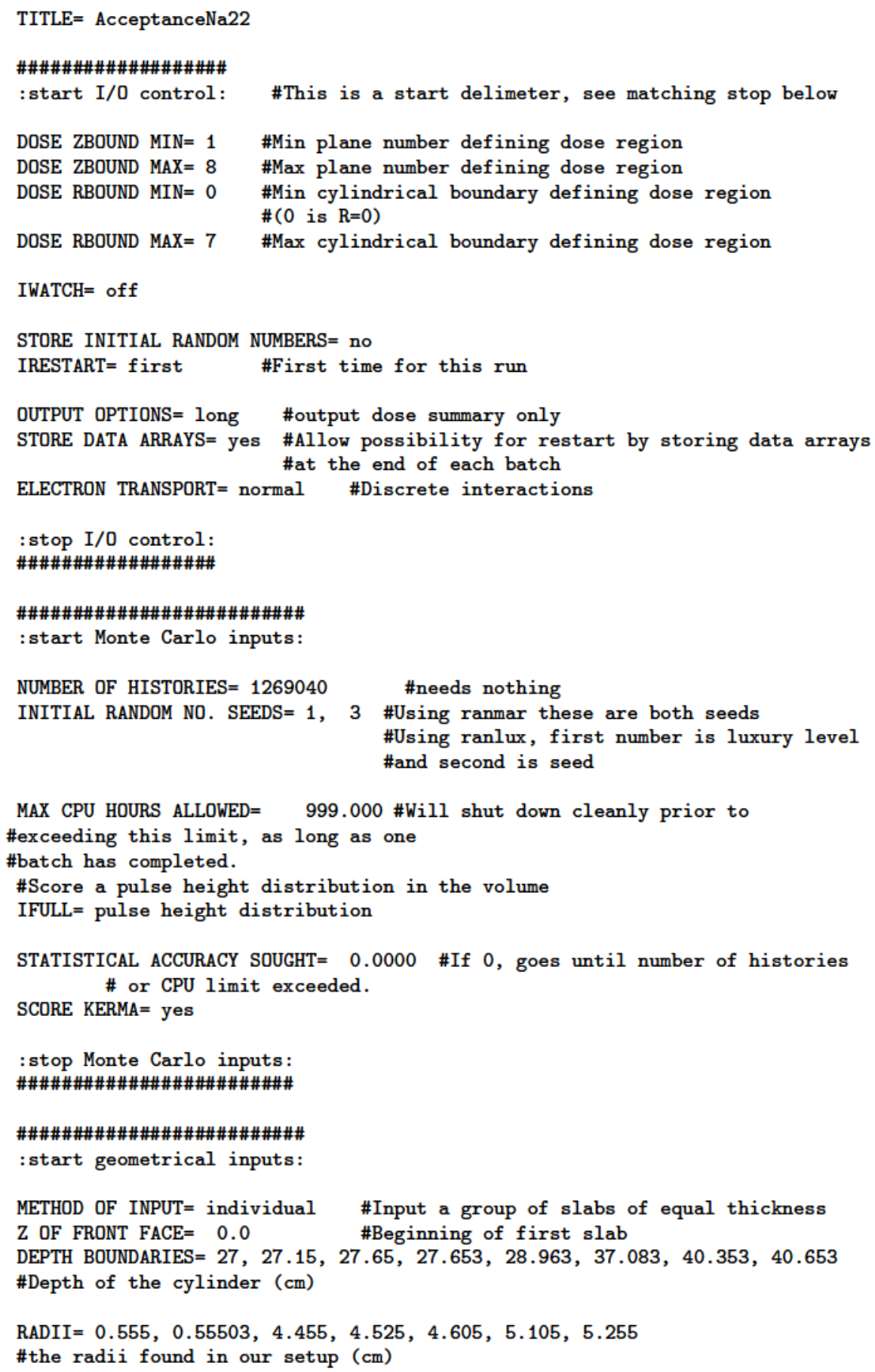




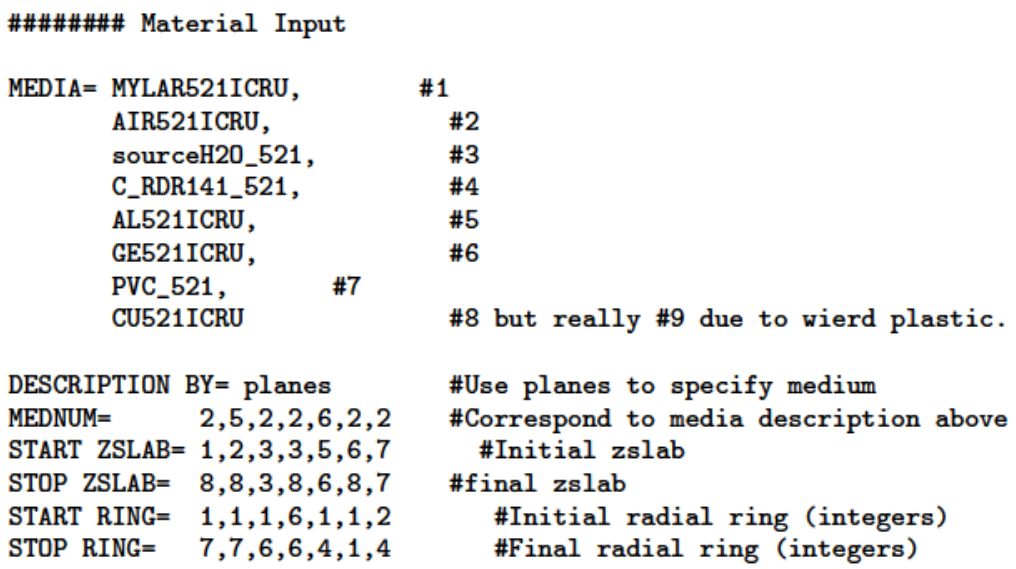

:stop geometrical inputs:

\#\#\#\#\#\#\#\#\#\#\#\#\#\#\#\#\#\#\#\#\#\#

\#\#\#\#\#\#\#\#\#\#\#\#\#\#\#\#\#\#\#\#\#\#\#\#\#\#\#\#\#\#\#\#

:start pulse height distribution input:

REGION OF SENSITIVE VOLUME= 6,14,22,23 \#Region numbers of sensitive volume

SLOTE $=-1$

DELTAE $=0.001$ \#Size of the energy bins in MeV \#...to analyze peak efficiencies. (default)

NUMBER OF BIN GROUPS $=50$

TOPS OF ENERGY BINS $1=0.001708984,0.003417968,0.005126952$, $0.006835936,0.008544920,0.010253904,0.011962888,0.013671872$, $0.015380856,0.017089840,0.018798824,0.020507808,0.022216792$, $0.023925776,0.025634760,0.027343744,0.029052728,0.030761712$, $0.032470696,0.034179680,0.035888664,0.037597648,0.039306632$, $0.041015616,0.042724600,0.044433584,0.046142568,0.047851552$, $0.049560536,0.051269520,0.052978504,0.054687488,0.056396472$;

TOPS OF ENERGY BINS $2=0.058105456,0.059814440,0.061523424$, $0.063232408,0.064941392,0.066650376,0.068359360,0.070068344$, $0.071777328,0.073486312,0.075195296,0.076904280,0.078613264$, $0.080322248,0.082031232,0.083740216,0.085449200,0.087158184$, $0.088867168,0.090576152,0.092285136,0.093994120,0.095703104$, $0.097412088,0.099121072,0.100830056,0.102539040,0.104248024$, $0.105957008,0.107665992,0.109374976,0.111083960,0.112792944$;

TOPS OF ENERGY BINS $3=0.114501928,0.116210912,0.117919896$, $0.119628880,0.121337864,0.123046848,0.124755832,0.126464816$, $0.128173800,0.129882784,0.131591768,0.133300752,0.135009736$, $0.136718720,0.138427704,0.140136688,0.141845672,0.143554656$, $0.145263640,0.146972624,0.148681608,0.150390592,0.152099576$, $0.153808560,0.155517544,0.157226528,0.158935512,0.160644496$, $0.162353480,0.164062464,0.165771448,0.167480432,0.169189416$;

TOPS OF ENERGY BINS $4=0.170898400,0.172607384,0.174316368$, $0.176025352,0.177734336,0.179443320,0.181152304,0.182861288$, $0.184570272,0.186279256,0.187988240,0.189697224,0.191406208$, $0.193115192,0.194824176,0.196533160,0.198242144,0.199951128$, $0.201660112,0.203369096,0.205078080,0.206787064,0.208496048$, $0.210205032,0.211914016,0.213623000,0.215331984,0.217040968$ $0.218749952,0.220458936,0.222167920,0.223876904,0.225585888$;

TOPS OF ENERGY BINS $5=0.227294872,0.229003856,0.230712840$, $0.232421824,0.234130808,0.235839792,0.237548776,0.239257760$, $0.240966744,0.242675728,0.244384712,0.246093696,0.247802680$, $0.249511664,0.251220648,0.252929632,0.254638616,0.256347600$, $0.258056584,0.259765568,0.261474552,0.263183536,0.264892520$, $0.266601504,0.268310488,0.270019472,0.271728456,0.273437440$, 
$0.275146424,0.276855408,0.278564392,0.280273376,0.281982360$;

TOPS OF ENERGY BINS $6=0.283691344,0.285400328,0.287109312$, $0.288818296,0.290527280,0.292236264,0.293945248,0.295654232$, $0.297363216,0.299072200,0.300781184,0.302490168,0.304199152$, $0.305908136,0.307617120,0.309326104,0.311035088,0.312744072$, $0.314453056,0.316162040,0.317871024,0.319580008,0.321288992$, $0.322997976,0.324706960,0.326415944,0.328124928,0.329833912$, $0.331542896,0.333251880,0.334960864,0.336669848,0.338378832$

TOPS OF ENERGY BINS $7=0.340087816,0.341796800,0.343505784$, $0.345214768,0.346923752,0.348632736,0.350341720,0.352050704$, $0.353759688,0.355468672,0.357177656,0.358886640,0.360595624$, $0.362304608,0.364013592,0.365722576,0.367431560,0.369140544$, $0.370849528,0.372558512,0.374267496,0.375976480,0.377685464$, $0.379394448,0.381103432,0.382812416,0.384521400,0.386230384$, $0.387939368,0.389648352,0.391357336,0.393066320,0.394775304$;

TOPS OF ENERGY BINS $8=0.396484288,0.398193272,0.399902256$, $0.401611240,0.403320224,0.405029208,0.406738192,0.408447176$, $0.410156160,0.411865144,0.413574128,0.415283112,0.416992096$, $0.418701080,0.420410064,0.422119048,0.423828032,0.425537016$, $0.427246000,0.428954984,0.430663968,0.432372952,0.434081936$, $0.435790920,0.437499904,0.439208888,0.440917872,0.442626856$, $0.444335840,0.446044824,0.447753808,0.449462792,0.451171776$;

TOPS OF ENERGY BINS $9=0.452880760,0.454589744,0.456298728$, $0.458007712,0.459716696,0.461425680,0.463134664,0.464843648$, $0.466552632,0.468261616,0.469970600,0.471679584,0.473388568$, $0.475097552,0.476806536,0.478515520,0.480224504,0.481933488$, $0.483642472,0.485351456,0.487060440,0.488769424,0.490478408$, $0.492187392,0.493896376,0.495605360,0.497314344,0.499023328$, $0.500732312,0.502441296,0.504150280,0.505859264,0.507568248$;

TOPS OF ENERGY BINS $10=0.509277232,0.510986216,0.512695200$, $0.514404184,0.516113168,0.517822152,0.519531136,0.521240120$, $0.522949104,0.524658088,0.526367072,0.528076056,0.529785040$, $0.531494024,0.533203008,0.534911992,0.536620976,0.538329960$, $0.540038944,0.541747928,0.543456912,0.545165896,0.546874880$, $0.548583864,0.550292848,0.552001832,0.553710816,0.555419800$, $0.557128784,0.558837768,0.560546752,0.562255736,0.563964720$;

TOPS OF ENERGY BINS $11=0.565673704,0.567382688,0.569091672$, $0.570800656,0.572509640,0.574218624,0.575927608,0.577636592$, $0.579345576,0.581054560,0.582763544,0.584472528,0.586181512$, $0.587890496,0.589599480,0.591308464,0.593017448,0.594726432$, $0.596435416,0.598144400,0.599853384,0.601562368,0.603271352$, $0.604980336,0.606689320,0.608398304,0.610107288,0.611816272$, $0.613525256,0.615234240,0.616943224,0.618652208,0.620361192$;

TOPS OF ENERGY BINS $12=0.622070176,0.623779160,0.625488144$, $0.627197128,0.628906112,0.630615096,0.632324080,0.634033064$, $0.635742048,0.637451032,0.639160016,0.640869000,0.642577984$, $0.644286968,0.645995952,0.647704936,0.649413920,0.651122904$, $0.652831888,0.654540872,0.656249856,0.657958840,0.659667824$, $0.661376808,0.663085792,0.664794776,0.666503760,0.668212744$, $0.669921728,0.671630712,0.673339696,0.675048680,0.676757664$;

TOPS OF ENERGY BINS $13=0.678466648,0.680175632,0.681884616$, $0.683593600,0.685302584,0.687011568,0.688720552,0.690429536$, $0.692138520,0.693847504,0.695556488,0.697265472,0.698974456$, $0.700683440,0.702392424,0.704101408,0.705810392,0.707519376$, $0.709228360,0.710937344,0.712646328,0.714355312,0.716064296$, $0.717773280,0.719482264,0.721191248,0.722900232,0.724609216$, $0.726318200,0.728027184,0.729736168,0.731445152,0.733154136$;

TOPS OF ENERGY BINS $14=0.734863120,0.736572104,0.738281088$, $0.739990072,0.741699056,0.743408040,0.745117024,0.746826008$, 
$0.748534992,0.750243976,0.751952960,0.753661944,0.755370928$, $0.757079912,0.758788896,0.760497880,0.762206864,0.763915848$, $0.765624832,0.767333816,0.769042800,0.770751784,0.772460768$, $0.774169752,0.775878736,0.777587720,0.779296704,0.781005688$, $0.782714672,0.784423656,0.786132640,0.787841624,0.789550608$

TOPS OF ENERGY BINS $15=0.791259592,0.792968576,0.794677560$, $0.796386544,0.798095528,0.799804512,0.801513496,0.803222480$, $0.804931464,0.806640448,0.808349432,0.810058416,0.811767400$, $0.813476384,0.815185368,0.816894352,0.818603336,0.820312320$, $0.822021304,0.823730288,0.825439272,0.827148256,0.828857240$, $0.830566224,0.832275208,0.833984192,0.835693176,0.837402160$, $0.839111144,0.840820128,0.842529112,0.844238096,0.845947080$;

TOPS OF ENERGY BINS $16=0.847656064,0.849365048,0.851074032$, $0.852783016,0.854492000,0.856200984,0.857909968,0.859618952$, $0.861327936,0.863036920,0.864745904,0.866454888,0.868163872$, $0.869872856,0.871581840,0.873290824,0.874999808,0.876708792$, $0.878417776,0.880126760,0.881835744,0.883544728,0.885253712$, $0.886962696,0.888671680,0.890380664,0.892089648,0.893798632$, $0.895507616,0.897216600,0.898925584,0.900634568,0.902343552$;

TOPS OF ENERGY BINS $17=0.904052536,0.905761520,0.907470504$, $0.909179488,0.910888472,0.912597456,0.914306440,0.916015424$, $0.917724408,0.9194333392,0.921142376,0.922851360,0.924560344$, $0.926269328,0.927978312,0.929687296,0.931396280,0.933105264$, $0.934814248,0.936523232,0.938232216,0.939941200,0.941650184$, $0.943359168,0.945068152,0.946777136,0.948486120,0.950195104$, $0.951904088,0.953613072,0.955322056,0.957031040,0.958740024$;

TOPS OF ENERGY BINS $18=0.960449008,0.962157992,0.963866976$, $0.965575960,0.967284944,0.968993928,0.970702912,0.972411896$, $0.974120880,0.975829864,0.977538848,0.979247832,0.980956816$, $0.982665800,0.984374784,0.986083768,0.987792752,0.989501736$, $0.991210720,0.992919704,0.994628688,0.996337672,0.998046656$, $0.999755640,1.001464624,1.003173608,1.004882592,1.006591576$, $1.008300560,1.010009544,1.011718528,1.013427512,1.015136496$;

TOPS OF ENERGY BINS $19=1.016845480,1.018554464,1.020263448$, $1.021972432,1.023681416,1.025390400,1.027099384,1.028808368$, $1.030517352,1.032226336,1.033935320,1.035644304,1.037353288$, $1.039062272,1.040771256,1.042480240,1.044189224,1.045898208$, $1.047607192,1.049316176,1.051025160,1.052734144,1.054443128$, $1.056152112,1.057861096,1.059570080,1.061279064,1.062988048$, $1.064697032,1.066406016,1.068115000,1.069823984,1.071532968$;

TOPS OF ENERGY BINS $20=1.073241952,1.074950936,1.076659920$, $1.078368904,1.080077888,1.081786872,1.083495856,1.085204840$, $1.086913824,1.088622808,1.090331792,1.092040776,1.093749760$, $1.095458744,1.097167728,1.098876712,1.100585696,1.102294680$, $1.104003664,1.105712648,1.107421632,1.109130616,1.110839600$, $1.112548584,1.114257568,1.115966552,1.117675536,1.119384520$, $1.121093504,1.122802488,1.124511472,1.126220456,1.127929440$;

TOPS OF ENERGY BINS $21=1.129638424,1.131347408,1.133056392$, $1.134765376,1.136474360,1.138183344,1.139892328,1.141601312$, $1.143310296,1.145019280,1.146728264,1.148437248,1.150146232$, $1.151855216,1.153564200,1.155273184,1.156982168,1.158691152$, $1.160400136,1.162109120,1.163818104,1.165527088,1.167236072$, $1.168945056,1.170654040,1.172363024,1.174072008,1.175780992$, $1.177489976,1.179198960,1.180907944,1.182616928,1.184325912$;

TOPS OF ENERGY BINS $22=1.186034896,1.187743880,1.189452864$, $1.191161848,1.192870832,1.194579816,1.196288800,1.197997784$, $1.199706768,1.201415752,1.203124736,1.204833720,1.206542704$, $1.208251688,1.209960672,1.211669656,1.213378640,1.215087624$, $1.216796608,1.218505592,1.220214576,1.221923560,1.223632544$, $1.225341528,1.227050512,1.228759496,1.230468480,1.232177464$, 
$1.233886448,1.235595432,1.237304416,1.239013400,1.240722384$;

TOPS OF ENERGY BINS $23=1.242431368,1.244140352,1.245849336$, $1.247558320,1.249267304,1.250976288,1.252685272,1.254394256$, $1.256103240,1.257812224,1.259521208,1.261230192,1.262939176$, $1.264648160,1.266357144,1.268066128,1.269775112,1.271484096$, $1.273193080,1.274902064,1.276611048,1.278320032,1.280029016$, $1.281738000,1.283446984,1.285155968,1.286864952,1.288573936$, $1.290282920,1.291991904,1.293700888,1.295409872,1.297118856$;

TOPS OF ENERGY BINS $24=1.298827840,1.300536824,1.302245808$, $1.303954792,1.305663776,1.307372760,1.309081744,1.310790728$, $1.312499712,1.314208696,1.315917680,1.317626664,1.319335648$, $1.321044632,1.322753616,1.324462600,1.326171584,1.327880568$, $1.329589552,1.331298536,1.333007520,1.334716504,1.336425488$, $1.338134472,1.339843456,1.341552440,1.343261424,1.344970408$, $1.346679392,1.348388376,1.350097360,1.351806344,1.353515328$;

TOPS OF ENERGY BINS $25=1.355224312,1.356933296,1.358642280$, $1.360351264,1.362060248,1.363769232,1.365478216,1.367187200$, $1.368896184,1.370605168,1.372314152,1.374023136,1.375732120$, $1.377441104,1.379150088,1.380859072,1.382568056,1.384277040$, $1.385986024,1.387695008,1.389403992,1.391112976,1.392821960$, $1.394530944,1.396239928,1.397948912,1.399657896,1.401366880$, $1.403075864,1.404784848,1.406493832,1.408202816,1.409911800$;

TOPS OF ENERGY BINS $26=1.411620784,1.413329768,1.415038752$, $1.416747736,1.418456720,1.420165704,1.421874688,1.423583672$, $1.425292656,1.427001640,1.428710624,1.430419608,1.432128592$, $1.433837576,1.435546560,1.437255544,1.438964528,1.440673512$, $1.442382496,1.444091480,1.445800464,1.447509448,1.449218432$, $1.450927416,1.452636400,1.454345384,1.456054368,1.457763352$, $1.459472336,1.461181320,1.462890304,1.464599288,1.466308272$;

TOPS OF ENERGY BINS $27=1.468017256,1.469726240,1.471435224$, $1.473144208,1.474853192,1.476562176,1.478271160,1.479980144$, $1.481689128,1.483398112,1.485107096,1.486816080,1.488525064$, $1.490234048,1.491943032,1.493652016,1.495361000,1.497069984$, $1.498778968,1.500487952,1.502196936,1.503905920,1.505614904$, $1.507323888,1.509032872,1.510741856,1.512450840,1.514159824$, $1.515868808,1.517577792,1.519286776,1.520995760,1.522704744$;

TOPS OF ENERGY BINS $28=1.524413728,1.526122712,1.527831696$, $1.529540680,1.531249664,1.532958648,1.534667632,1.536376616$, $1.538085600,1.539794584,1.541503568,1.543212552,1.544921536$, $1.546630520,1.548339504,1.550048488,1.551757472,1.553466456$, $1.555175440,1.556884424,1.558593408,1.560302392,1.562011376$, $1.563720360,1.565429344,1.567138328,1.568847312,1.570556296$, $1.572265280,1.573974264,1.575683248,1.577392232,1.579101216$;

TOPS OF ENERGY BINS $29=1.580810200,1.582519184,1.584228168$, $1.585937152,1.587646136,1.589355120,1.591064104,1.592773088$, $1.594482072,1.596191056,1.597900040,1.599609024,1.601318008$, $1.603026992,1.604735976,1.606444960,1.608153944,1.609862928$, $1.611571912,1.613280896,1.614989880,1.616698864,1.618407848$, $1.620116832,1.621825816,1.623534800,1.625243784,1.626952768$, $1.628661752,1.630370736,1.632079720,1.633788704,1.635497688$;

TOPS OF ENERGY BINS $30=1.637206672,1.638915656,1.640624640$, $1.642333624,1.644042608,1.645751592,1.647460576,1.649169560$, $1.650878544,1.652587528,1.654296512,1.656005496,1.657714480$, $1.659423464,1.661132448,1.662841432,1.664550416,1.666259400$, $1.667968384,1.669677368,1.671386352,1.673095336,1.674804320$, $1.676513304,1.678222288,1.679931272,1.681640256,1.683349240$, $1.685058224,1.686767208,1.688476192,1.690185176,1.691894160$;

TOPS OF ENERGY BINS $31=1.693603144,1.695312128,1.697021112$, $1.698730096,1.700439080,1.702148064,1.703857048,1.705566032$, 
$1.707275016,1.708984000,1.710692984,1.712401968,1.714110952$, $1.715819936,1.717528920,1.719237904,1.720946888,1.722655872$, $1.724364856,1.726073840,1.727782824,1.729491808,1.731200792$, $1.732909776,1.734618760,1.736327744,1.738036728,1.739745712$, $1.741454696,1.743163680,1.744872664,1.746581648,1.748290632$

TOPS OF ENERGY BINS $32=1.749999616,1.751708600,1.753417584$, $1.755126568,1.756835552,1.758544536,1.760253520,1.761962504$, $1.763671488,1.765380472,1.767089456,1.768798440,1.770507424$, $1.772216408,1.773925392,1.775634376,1.777343360,1.779052344$, $1.780761328,1.782470312,1.784179296,1.785888280,1.787597264$, $1.789306248,1.791015232,1.792724216,1.794433200,1.796142184$, $1.797851168,1.799560152,1.801269136,1.802978120,1.804687104$;

TOPS OF ENERGY BINS $33=1.806396088,1.808105072,1.809814056$, $1.811523040,1.813232024,1.814941008,1.816649992,1.818358976$, $1.820067960,1.821776944,1.823485928,1.825194912,1.826903896$, $1.828612880,1.830321864,1.832030848,1.833739832,1.835448816$, $1.837157800,1.838866784,1.840575768,1.842284752,1.843993736$, $1.845702720,1.847411704,1.849120688,1.850829672,1.852538656$, $1.854247640,1.855956624,1.857665608,1.859374592,1.861083576$;

TOPS OF ENERGY BINS $34=1.862792560,1.864501544,1.866210528$, $1.867919512,1.869628496,1.871337480,1.873046464,1.874755448$, $1.876464432,1.878173416,1.879882400,1.881591384,1.883300368$, $1.885009352,1.886718336,1.888427320,1.890136304,1.891845288$, $1.893554272,1.895263256,1.896972240,1.898681224,1.900390208$, $1.902099192,1.903808176,1.905517160,1.907226144,1.908935128$, $1.910644112,1.912353096,1.914062080,1.915771064,1.917480048$;

TOPS OF ENERGY BINS $35=1.919189032,1.920898016,1.922607000$, $1.924315984,1.926024968,1.927733952,1.929442936,1.931151920$, $1.932860904,1.934569888,1.936278872,1.937987856,1.939696840$, $1.941405824,1.943114808,1.944823792,1.946532776,1.948241760$, $1.949950744,1.951659728,1.953368712,1.955077696,1.956786680$, $1.958495664,1.960204648,1.961913632,1.963622616,1.965331600$, $1.967040584,1.968749568,1.970458552,1.972167536,1.973876520$;

TOPS OF ENERGY BINS $36=1.975585504,1.977294488,1.979003472$, $1.980712456,1.982421440,1.984130424,1.985839408,1.987548392$, $1.989257376,1.990966360,1.992675344,1.994384328,1.996093312$, $1.997802296,1.999511280,2.001220264,2.002929248,2.004638232$, $2.006347216,2.008056200,2.009765184,2.011474168,2.013183152$, $2.014892136,2.016601120,2.018310104,2.020019088,2.021728072$, $2.023437056,2.025146040,2.026855024,2.028564008,2.030272992$;

TOPS OF ENERGY BINS $37=2.031981976,2.033690960,2.035399944$, $2.037108928,2.038817912,2.040526896,2.042235880,2.043944864$, $2.045653848,2.047362832,2.049071816,2.050780800,2.052489784$, $2.054198768,2.055907752,2.057616736,2.059325720,2.061034704$, $2.062743688,2.064452672,2.066161656,2.067870640,2.069579624$, $2.071288608,2.072997592,2.074706576,2.076415560,2.078124544$, $2.079833528,2.081542512,2.083251496,2.084960480,2.086669464$;

TOPS OF ENERGY BINS $38=2.088378448,2.090087432,2.091796416$, $2.093505400,2.095214384,2.096923368,2.098632352,2.100341336$, $2.102050320,2.103759304,2.105468288,2.107177272,2.108886256$, $2.110595240,2.112304224,2.114013208,2.115722192,2.117431176$, $2.119140160,2.120849144,2.122558128,2.124267112,2.125976096$, $2.127685080,2.129394064,2.131103048,2.132812032,2.134521016$, $2.136230000,2.137938984,2.139647968,2.141356952,2.143065936$;

TOPS OF ENERGY BINS $39=2.144774920,2.146483904,2.148192888$, $2.149901872,2.151610856,2.153319840,2.155028824,2.156737808$, $2.158446792,2.160155776,2.161864760,2.163573744,2.165282728$, $2.166991712,2.168700696,2.170409680,2.172118664,2.173827648$, $2.175536632,2.177245616,2.178954600,2.180663584,2.182372568$, $2.184081552,2.185790536,2.187499520,2.189208504,2.190917488$, 
$2.192626472,2.194335456,2.196044440,2.197753424,2.199462408$;

TOPS OF ENERGY BINS $40=2.201171392,2.202880376,2.204589360$, $2.206298344,2.208007328,2.209716312,2.211425296,2.213134280$, $2.214843264,2.216552248,2.218261232,2.219970216,2.221679200$, $2.223388184,2.225097168,2.226806152,2.228515136,2.230224120$, $2.231933104,2.233642088,2.235351072,2.237060056,2.238769040$, $2.240478024,2.242187008,2.243895992,2.245604976,2.247313960$, $2.249022944,2.250731928,2.252440912,2.254149896,2.255858880$;

TOPS OF ENERGY BINS $41=2.257567864,2.259276848,2.260985832$, $2.262694816,2.264403800,2.266112784,2.267821768,2.269530752$, $2.271239736,2.272948720,2.274657704,2.276366688,2.278075672$, $2.279784656,2.281493640,2.283202624,2.284911608,2.286620592$, $2.288329576,2.290038560,2.291747544,2.293456528,2.295165512$, $2.296874496,2.298583480,2.300292464,2.302001448,2.303710432$, $2.305419416,2.307128400,2.308837384,2.310546368,2.312255352$;

TOPS OF ENERGY BINS $42=2.313964336,2.315673320,2.317382304$, $2.319091288,2.320800272,2.322509256,2.324218240,2.325927224$, $2.327636208,2.329345192,2.331054176,2.332763160,2.334472144$, $2.336181128,2.337890112,2.339599096,2.341308080,2.343017064$, $2.344726048,2.346435032,2.348144016,2.349853000,2.351561984$, $2.353270968,2.354979952,2.356688936,2.358397920,2.360106904$, $2.361815888,2.363524872,2.365233856,2.366942840,2.368651824$;

TOPS OF ENERGY BINS $43=2.370360808,2.372069792,2.373778776$, $2.375487760,2.377196744,2.378905728,2.380614712,2.382323696$, $2.384032680,2.385741664,2.387450648,2.389159632,2.390868616$, $2.392577600,2.394286584,2.395995568,2.397704552,2.399413536$, $2.401122520,2.402831504,2.404540488,2.406249472,2.407958456$, $2.409667440,2.411376424,2.413085408,2.414794392,2.416503376$, $2.418212360,2.419921344,2.421630328,2.423339312,2.425048296$;

TOPS OF ENERGY BINS $44=2.426757280,2.428466264,2.430175248$, $2.431884232,2.433593216,2.435302200,2.437011184,2.438720168$, $2.440429152,2.442138136,2.443847120,2.445556104,2.447265088$, $2.448974072,2.450683056,2.452392040,2.454101024,2.455810008$, $2.457518992,2.459227976,2.460936960,2.462645944,2.464354928$, $2.466063912,2.467772896,2.469481880,2.471190864,2.472899848$, $2.474608832,2.476317816,2.478026800,2.479735784,2.481444768$;

TOPS OF ENERGY BINS $45=2.483153752,2.484862736,2.486571720$, $2.488280704,2.489989688,2.491698672,2.493407656,2.495116640$, $2.496825624,2.498534608,2.500243592,2.501952576,2.503661560$, $2.505370544,2.507079528,2.508788512,2.510497496,2.512206480$, $2.513915464,2.515624448,2.517333432,2.519042416,2.520751400$, $2.522460384,2.524169368,2.525878352,2.527587336,2.529296320$, $2.531005304,2.532714288,2.534423272,2.536132256,2.537841240$;

TOPS OF ENERGY BINS $46=2.539550224,2.541259208,2.542968192$, $2.544677176,2.546386160,2.548095144,2.549804128,2.551513112$, $2.553222096,2.554931080,2.556640064,2.558349048,2.560058032$, $2.561767016,2.563476000,2.565184984,2.566893968,2.568602952$, $2.570311936,2.572020920,2.573729904,2.575438888,2.577147872$, $2.578856856,2.580565840,2.582274824,2.583983808,2.585692792$, $2.587401776,2.589110760,2.590819744,2.592528728,2.594237712$;

TOPS OF ENERGY BINS $47=2.595946696,2.597655680,2.599364664$, $2.601073648,2.602782632,2.604491616,2.606200600,2.607909584$, $2.609618568,2.611327552,2.613036536,2.614745520,2.616454504$, $2.618163488,2.619872472,2.621581456,2.623290440,2.624999424$, $2.626708408,2.628417392,2.630126376,2.631835360,2.633544344$, $2.635253328,2.636962312,2.638671296,2.640380280,2.642089264$ $2.643798248,2.645507232,2.647216216,2.648925200,2.650634184$;

TOPS OF ENERGY BINS $48=2.652343168,2.654052152,2.655761136$, $2.657470120,2.659179104,2.660888088,2.662597072,2.664306056$, 
$2.666015040,2.667724024,2.669433008,2.671141992,2.672850976$, $2.674559960,2.676268944,2.677977928,2.679686912,2.681395896$, $2.683104880,2.684813864,2.686522848,2.688231832,2.689940816$, $2.691649800,2.693358784,2.695067768,2.696776752,2.698485736$, $2.700194720,2.701903704,2.703612688,2.705321672,2.707030656$;

TOPS OF ENERGY BINS $49=2.708739640,2.710448624,2.712157608$, $2.713866592,2.715575576,2.717284560,2.718993544,2.720702528$, $2.722411512,2.724120496,2.725829480,2.727538464,2.729247448$, $2.730956432,2.732665416,2.734374400,2.736083384,2.737792368$, $2.739501352,2.741210336,2.742919320,2.744628304,2.746337288$, $2.748046272,2.749755256,2.751464240,2.753173224,2.754882208$, $2.756591192,2.758300176,2.760009160,2.761718144,2.763427128$;

TOPS OF ENERGY BINS $50=2.765136112,2.766845096,2.768554080$, $2.770263064,2.771972048,2.773681032,2.775390016,2.777099000$, $2.778807984,2.780516968,2.782225952,2.783934936,2.785643920$, $2.787352904,2.789061888,2.790770872,2.792479856,2.794188840$, $2.795897824,2.797606808,2.799315792,2.801024776,2.802733760$, $2.804442744,2.806151728,2.807860712,2.809569696,2.811278680$, $2.812987664,2.814696648,2.816405632,2.818114616,2.819823600$;

:stop pulse height distribution input:

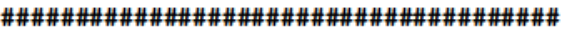

\#\#\#\#\#\#\#\#\#\#\#\#\#\#\#\#\#\#

:start source inputs:

INCIDENT PARTICLE $=$ photon SOURCE NUMBER $=1$

\#from front

\#SOURCE NUMBER $=3$

\#Point source on axis incident

SOURCE OPTIONS $=0,5.255,0,0$

\#Internal uniform isotropically

\#radiating disk of infinite size \#SOURCE OPTIONS $=27,5.255,0,0$ \#SOURCE OPTIONS $=0,3.1215,1.27,5.728$

\#No inputs here for this source-\#broad beam source uses equivalence \#between depth-dose in small radius \#on central axis due to broad beam \#and depth-dose in large radius due \#to pencil beam--thus broad beam is \#in fact a pencil beam $(\mathrm{r}=0 \mathrm{~cm})$ \#incident on a phantom of radius $\# 1000 \mathrm{~cm}$ (overrides user-input radius) \#oh yeah, the broad beam is always \#has $\mathrm{X}, \mathrm{Y}, \mathrm{Z}$ direction cosines

INCIDENT ENERGY= monoenergetic $\#(0,0,1)$

:stop source inputs:

\#\#\#\#\#\#\#\#\#\#\#\#\#\#\#\#\#\#\#\#\#\#\#

\#\#\#\#\#\#\#\#\#\#\#\#\#\#\#\#\#\#\#\#\#\#\#

:Start MC Transport Parameter:

Global ECUT=

Global PCUT $=$

Global SMAX= \#unless old PRESTA algorithm used) ESTEPE=

$\mathrm{X}$ IMAX $=$

Boundary crossing algorithm=
0.521 \#Electron cutoff for transport 0.01 \#Photon cutoff for transport 1 e10 \#aximum step size in $\mathrm{cm}$ (not needed 0.25 \#Max fractional continuous energy loss \#per step. Use 0.25 unless using \#PRESTA-I

0.5 \#Max first elastic scattering moment \#per step. Using default.

exact \#Cross boundaries in single scattering 


\begin{tabular}{|c|c|c|}
\hline & & \#mode \\
\hline Skin depth for $B C A=$ & 3 & $\begin{array}{l}\text { \#Distance from a boundary (in elastic } \\
\text { \#MFP) at which the algorithm will go } \\
\text { \#into single scattering mode (using } \\
\text { \#default here) }\end{array}$ \\
\hline Electron-step algorithm $=$ PRESTA & -II & \#Use new physics \\
\hline Spin effects $=$ & On & $\begin{array}{l}\text { \#Include spin effects for electron } \\
\text { \#elastic scattering }\end{array}$ \\
\hline Brems angular sampling= & KM & $\begin{array}{l}\text { \#Modified KM used to determine } \\
\text { \#emission angle of bremsstrahlung } \\
\text { \#photons }\end{array}$ \\
\hline Brems cross sections= & BH & \#Use Bethe-Heitler cross-sections \\
\hline Bound Compton scattering= & On & \#Use Klein Nishina for Compton \\
\hline Pair angular sampling= & KM & $\begin{array}{l}\text { \#Use leading term to determine } \\
\text { \#angle of pairs }\end{array}$ \\
\hline Photoelectron angular sampling= & On & $\begin{array}{l}\text { \#Photoelectrons get direction of } \\
\text { \#photon that creates them }\end{array}$ \\
\hline Rayleigh scattering= & Off & \#No coherent scattering \\
\hline Atomic relaxations $=$ & On & $\begin{array}{l}\text { \#Simulate emission of fluorescent } \\
\text { \#X-rays, Auger and Coster-Kronig } \\
\text { \#electrons }\end{array}$ \\
\hline
\end{tabular}

:Stop MC Transport Parameter:

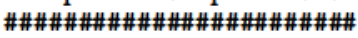

:Start Variance Reduction:

BREM SPLITTING = Off \#No bremsstrahlung splitting

NUMBER OF BREMS PER EVENT=1 \#0nly used with bremsstrahlung splitting

CHARGED PARTICLE RUSSIAN ROULETTE $=$ Off

ELECTRON RANGE REJECTION= off \#No range rejection

ESAVEIN $=0.0 \quad$ \#0nly used with range rejection

RUSSIAN ROULETTE DEPTH $=0.0000$ \#No photon Russian Roulette

RUSSIAN ROULETTE FRACTION= 0.0000 \#No photon Russian Roulette

EXPONENTIAL TRANSFORM C $=0.0000$ \#No pathlength biasing

PHOTON FORCING = off \#Force photons to interact in geometry

START FORCING= 1 \#Start forcing at first interaction

STOP FORCING AFTER= 1 \#0nly force one interaction/history

CS ENHANCEMENT FACTOR=1.0 \#Photon cross sections scaling factor

CS ENHANCEMENT START REGION=1 \#Region outside geometry--> no cross-

\#section scaling

CS ENHANCEMENT STOP REGION= 1 \#Region outside geometry--> no cross-

\#section scaling

:Stop Variance Reduction:

\#\#\#\#\#\#\#\#\#\#\#\#\#\#\#\#\#\#\#\#\#

:start plot control:

PLOTTING $=$ on

EXTERNAL PLOTTER OUTPUT $=$ On \#Create .plotdat file

EXTERNAL PLOT TYPE= both \#Type of .plotdat file

PLOT RADIAL REGION IX=1 \#Plot central axis depth-dose curve

PLOT PLANAR REGION IZ $=2$ \#No dose vs radius plots

:stop plot control:

\#\#\#\#\#\#\#\#\#\#\#\#\#\#\#\#\#\#\#\#\# 